\title{
Exploring Applicability of Direct Analysis in Real Time with Mass Spectrometry (DART-MS) to Identify Homemade Explosive Residues Post-Blast
}

by

Chelsea Elizabeth Black

A thesis submitted to the Faculty of Graduate and Postdoctoral Affairs in partial fulfillment of the requirements for the degree of

\author{
Master of Science \\ In \\ Chemistry \\ Carleton University \\ Ottawa, Ontario
}

(C) 2019

Chelsea Elizabeth Black 


\section{Abstract}

Application of Direct-Analysis-in-Real-Time (DART) ionization with mass spectrometry (DART-MS) to identify explosives from post-blast residues is presented. Explosives of interest represent real current threats encountered in criminal investigations in North America and Europe: homemade organic peroxides, binary explosives and smokeless powder. A series of simulated improvised explosive devices (IEDs) were manufactured using triacetone triperoxide (TATP), hexamethylene triperoxide diamine (HMTD), methyl ethyl ketone peroxide (MEKP), homemade binary explosives (composed of a fuel-oxidizer) and single and double-base smokeless powders. Each IED was configured to yield bomb fragments representative of actual materials recovered from bombing investigations. The goal of this study was to demonstrate the validity of DART-MS for identification of homemade explosives using real world samples (i.e. not laboratory simulations) and develop a quality assured method for use in accredited forensic laboratory settings. Smokeless powder was of specific interest as there is currently no reported method to identify nitrocellulose (NC) post-blast, unless unconsumed material is recovered. Therefore, this study aimed to demonstrate the validity of DART-MS to characterize thermal breakdown products of NC. All recovered fragments were analyzed directly and in directly (i.e. cotton swabs and solvent extraction methods) using full scan high resolution mass spectrometry (HRMS). This work demonstrates the forensic validity of DART-MS to provide rapid and quality assured identification of explosive residues from real post-blast IED fragments. 


\section{Acknowledgements}

To my supervisors Dr. Nigel Hearns and Dr. Jeffrey Smith, I would like to thank you both for the opportunity to work on this project with you both. Your vision, support and guidance throughout our working years together was paramount in the success of this project. Jeff, I truly appreciate the amazing opportunities you have given me. For all the advice, support and encouragement, I am truly thankful. Having come to your office in both moments of excitement and stress, you always knew exactly what I needed to hear to further motivate me or help me get through any struggles I was experiencing. Nigel, I am forever grateful for the experience I have had working on this project with you. The development of my knowledge and skills are a direct result of the patience and time you have taken teaching when me about forensic science, explosives, experimental design, professionalism and the list goes on. Thank you for advocating for this research within the lab and the organization. Your continuous, never-ending support has made this the most rewarding and memorable working experience of my life. Thank-you!

To my friends and family - I would like to thank you all for your continuous encouragement and support during the completion of my Masters. Dad, with the turning of each school year, and yes I know there have been many, your support has never wavered. As a parent chaperone on public school field trips, a fan in the stands of many sports games and coming to Ottawa to sit in as a special guest at my defence - you have never missed any special moment in my educational career, big or small. Your understanding and appreciation for my love of school has allowed me to pursue any and every dream I have ever had. Your kind words, immense love and thoughtful advice has 
helped me reach each destination in the path that has led me to the end of grad school. You are truly my greatest confidant. Mom, having shared your innate love for learning with me, I have you to thank for my drive and determination to obtain such a fulsome education. Ryan and Courtney, you have been the greatest role-models a little sister could ask for. Your hard-work, determination, and commitment to your professions while maintaining important relationships and friendships is something I truly admire in both of you. Thank you so much for always cheering me on, providing sound advice and encouraging me to never give up! To the MacDonald family, I can never thank you enough for including me in your extremely loving and supportive family. Finally, to my girls Christine, Stephanie, Annah, Jillian and Kylie. Our friendship means the world to me. I am so thankful for all the memories made and your never-ending support and encouragement.

Being a member of the Smith Lab has been such a pleasure. Participating in group meetings, celebrating the holidays at our epic Christmas parties and sharing a glass of beer (cider for me of course (:) at the end of the week will forever be some of my fondest memories of my time at Carleton University. To all Smith lab members, current and alumni, I want to thank you for the knowledge, experience and advice you have shared with me during my time in the lab. Karl, our ability to naturally segue from experiment troubleshooting to our latest Friday night adventures with our friends and families will never be lost upon me. Our friendship is extremely important to me and I look forward to continuous updates and catching up when you come to visit in Kingston! 
To all the members of the Department of Chemistry at Carleton University, I thank you for your support, knowledge, ideas and friendships and the many learning and teaching opportunities provided to me by TA positions and volunteer opportunities.

To all the members of the Trace Evidence section of the Forensic Laboratory Services and the RCMP at large; I thank you for your support, access to resources, knowledge and patience as I navigated my way through this research study. You have been so welcoming, encouraging and supportive. 


\section{Table of Contents}

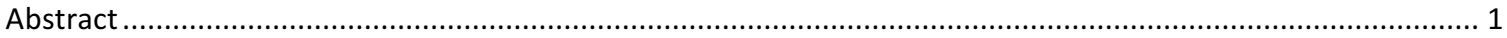

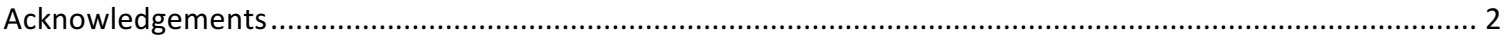

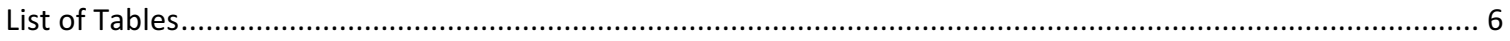

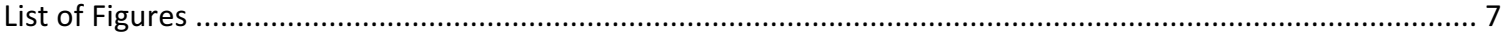

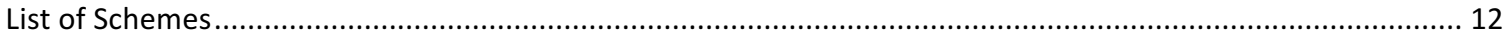

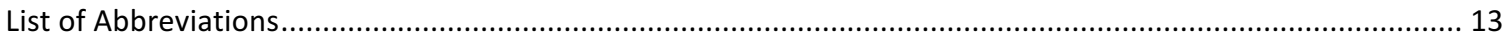

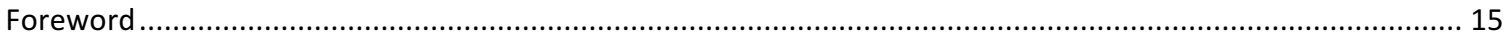

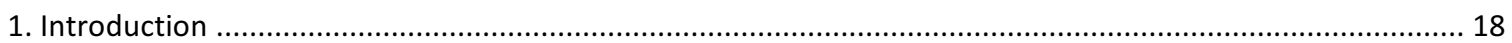

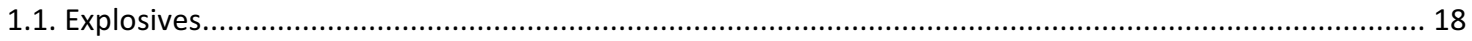

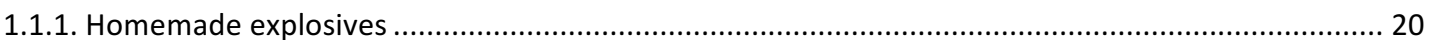

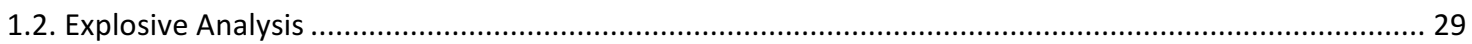

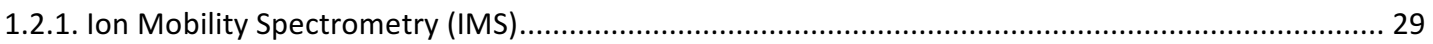

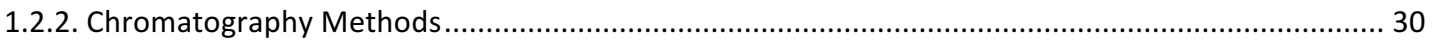

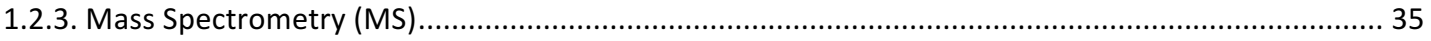

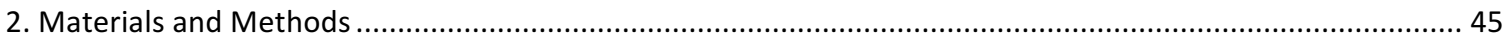

2.1. Consumables, Reagents and Standard Reference Materials .........................................................46

2.2. IED Construction, Detonation and Fragment Collection............................................................ 47

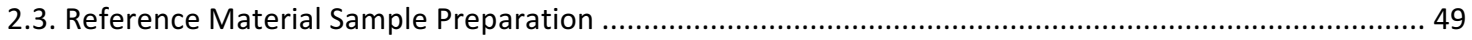

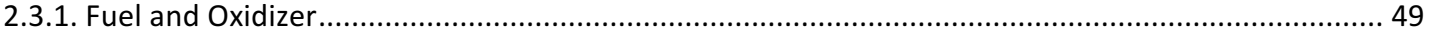

2.3.2. Octanitrate Cellobiose Synthesis and Sample Preparation ..........................................................49

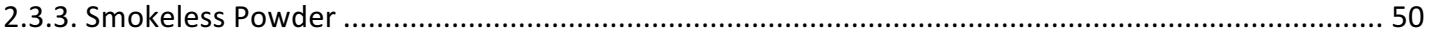

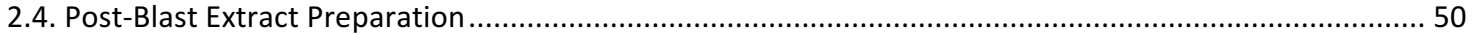

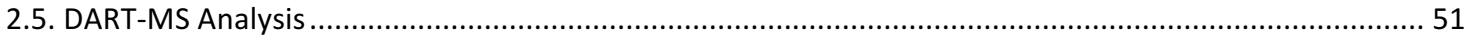

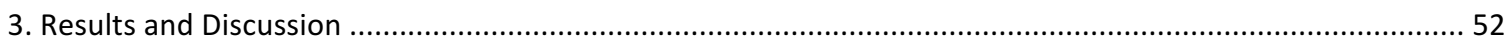

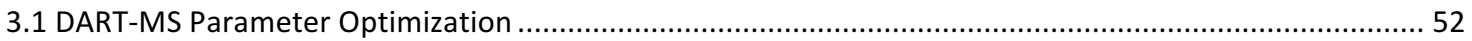

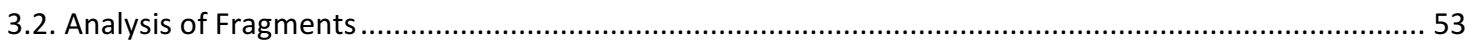

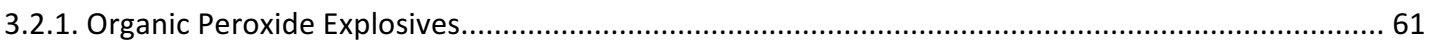

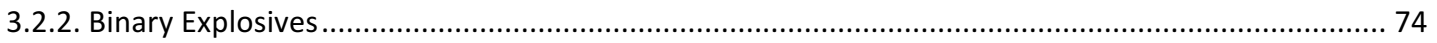

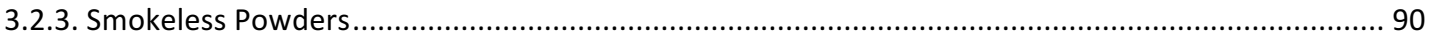

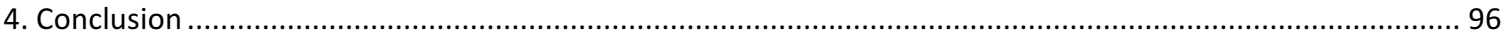

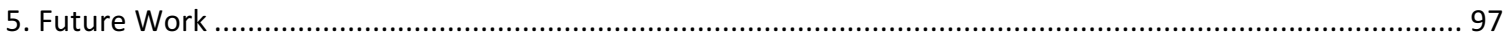

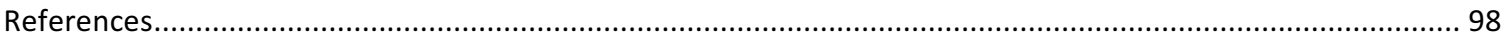

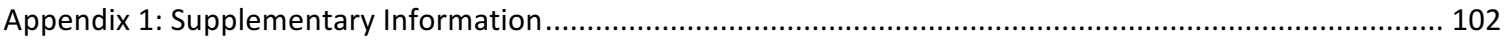




\section{List of Tables}

Table 1. Examples of products that source components for binary explosives. ...........................24

Table 2. Type and amount of explosive used as main charge for each IED ................................ 48

Table 3. LODs observed for explosives of interest measured using in-house method and QuickStrip compared to literature values.

Table 4. Depiction of the OPBE identified via DART-MS direct analysis of a multitude of different post-blast fragments compared to in-direct.

Table 5. Characteristic ions of HMTD present upon analysis of residues collected via swabs differentiated by substrate.

Table 6. List of mass formulae for the ions characteristic of nitrated sugar thermal breakdown products, with associated mass shift (amu). 


\section{List of Figures}

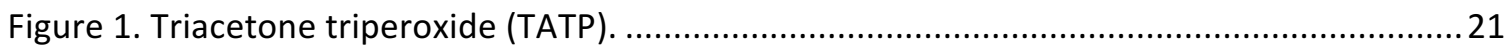

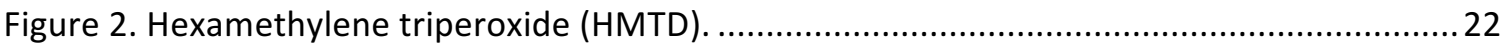

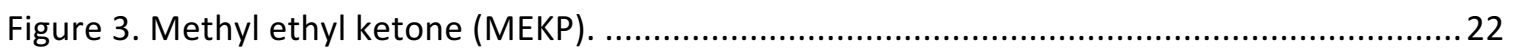

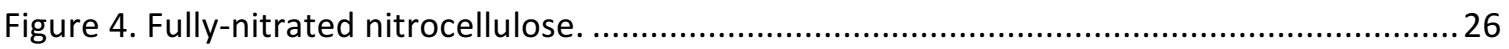

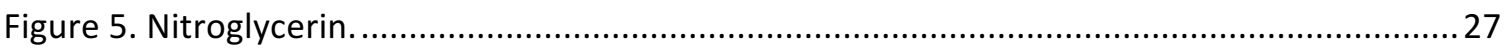

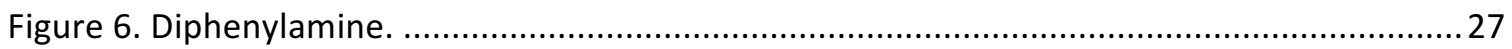

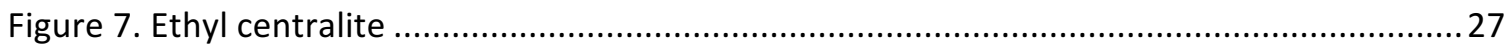

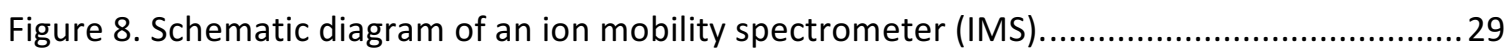

Figure 9. Schematic diagram of the instrumentation used to separate analytes of a mixture via high-performance liquid chromatography (HPLC). ........................................................ 31

Figure 10. Schematic diagram of the instrumentation used to separate volatile components in a

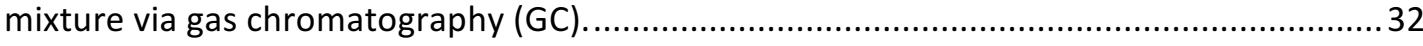

Figure 11. Schematic diagram of the instrumentation used for simultaneous separation of anions

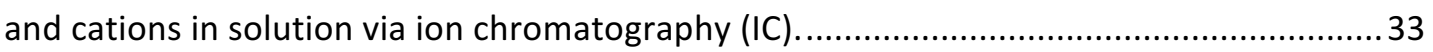

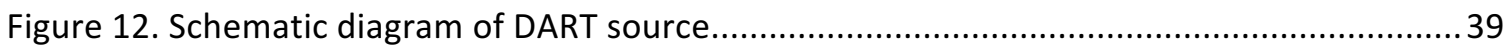

Figure 13. Images of the different orientations of the DART source with respect to the MS interface: (a) surface desorption mode and (b) transmission mode....................................42

Figure 14. Schematic diagram of the design and engineering of the Q-Exactive hybrid mass spectrometer

Figure 15. Fragments collected post-blast from the detonation of devices utilizing homemade explosives as the main charge (IED\# 1-14).

Figure 16. Ions observed via operation of the QExactive in positive full scan mode, without the

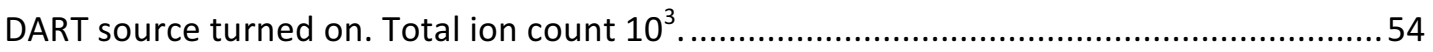

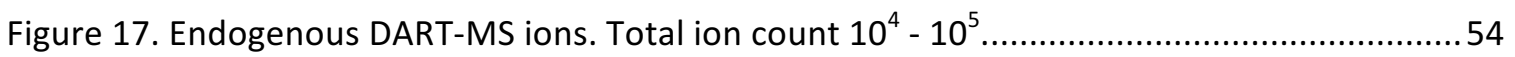

Figure 18. Analysis of an unused cotton swab in positive mode using full scan. Total ion count $10^{4}$

Figure 19. An unused QuickStrip analyzed in positive mode using full scan. Total ion count $10^{3}$ $10^{5}$. 
Figure 20. Water deposited onto a QuickStrip, analyzed in positive mode using full scan. Total ion count $10^{4}-10^{5}$.

Figure 21. Methanol deposited onto a QuickStrip, analyzed in positive mode using full scan. Total ion count $10^{4}-10^{6}$. 56

Figure 22. Acetone deposited onto a QuickStrip, analyzed in positive mode using full scan. Total ion count $10^{4}-10^{6}$.

Figure 23. Acetonitrile deposited onto a QuickStrip, analzyed in positive mode using full scan. Total ion count $10^{4}-10^{6}$.

Figure 24. Analysis of dichloromethane deposited onto a QuickStrip, in positive mode using full scan, to be used for solvent associated ion subtraction. Total ion count $10^{4}-10^{6}$.

Figure 25. Analysis of hexane deposited onto a QuickStrip, in positive mode using full scan. Total ion count $10^{4}-10^{5}$.

Figure 26. Positive mode, full scan high-resolution mass spectrum for TATP analyzed from certified reference standard. lons characteristic of TATP have been bolded and labelled... 63

Figure 27. Positive mode, full scan high-resolution mass spectrum for TATP upon direct analysis of fragment from IED \#2. Ions characteristic of TATP have been bolded and labelled.........64

Figure 28. Positive mode, full scan high-resolution mass spectrum for TATP analyzed from a swab used to collect post-blast residues from IED \#2 fragments. lons characteristic of TATP have been bolded and labelled.

Figure 29.Full scan high-resolution mass spectrum for HMTD analyzed from certified reference. lons characteristic of HMTD have been bolded and labelled 65

Figure 30. Full scan high-resolution mass spectrum depicting identification of HMTD from direct analysis of a fragment collected post-blast from IED \#3. Ions characteristic of HMTD have been bolded and labelled.

Figure 31. Full scan high-resolution mass spectrum depicting identification of HMTD upon analysis of a swab used to collect post-blast residues from IED \#3 fragments. Ions characteristic of HMTD have been bolded and labelled.

Figure 32. Positive mode, full scan high-resolution mass spectrum for MEKP analyzed from the crude synthesized product. Ions characteristic of MEKP have been bolded and labelled... 68

Figure 33. Positive mode, full scan high-resolution mass spectrum depicting identification of MEKP from direct analysis of fragment collected post-blast from IED \#5. Ions characteristic of MEKP have been bolded and labelled. 
Figure 34. Positive mode, full scan high-resolution mass spectrum depicting identification of MEKP upon analysis of a swab used to collected post-blast residues from IED \#5 fragments. Ions characteristic of MEKP have been bolded and labelled.

Figure 35. From left to right - cotton swab, paper swab, modified paper swab. 71

Figure 36. Full scan high-resolution mass spectra depicting identification of HMTD upon collection of post-blast residues from IED \#5 using dry and solvent dampened swabs. ...... 73

Figure 37. Full scan high-resolution mass spectrum of glucose dissolved in water as a reference material. Collected in positive mode.

Figure 38. Full scan high- resolution mass spectrum of sucrose dissolved in water, used as a reference material. Collected in positive mode.

Figure 39. Full scan high-resolution mass spectrum of TANG dissolved in water, used as a reference material. Collected in positive mode.

Figure 40. Full scan high-resolution mass spectrum depicting identification of a glucose containing product from post-blast residues extracted with water from IED \#11 metal substrate fragment. Collected in positive mode.

Figure 41. Full scan high-resolution mass spectrum depicting identification of a glucose containing product from post-blast residues extracted with water from IED \#11 plastic substrate fragment. Collected in positive mode.

Figure 42. Full scan high-resolution mass spectrum depicting identification of a glucose containing product from post-blast residues extracted with water from IED \#11 rubber substrate fragment. Collected in positive mode.

Figure 43. Full scan high- resolution mass spectrum of dextrin reference material dissolved in water. Collected in positive mode.

Figure 44. Full scan high-resolution mass spectrum depicting identification of a glucose containing product from post-blast residues extracted with water from IED \#12 plastic substrate fragment. Collected in positive mode.

Figure 45. Full scan high-resolution mass spectrum depicting identification of a glucose containing product from post-blast residues extracted with water from IED \#12 metal substrate fragment. Collected in positive mode.

Figure 46. Full scan high-resolution mass spectrum depicting identification of a glucose containing product from post-blast residues extracted with water from IED \#12 rubber substrate fragment. Collected in positive mode. 
Figure 47 Full scan high-resolution mass spectrum of an automotive grease, used as a reference material. Collected in positive mode.

Figure 48. Full scan high-resolution mass spectrum depicting identification of automotive grease from post-blast residues extracted with hexane from IED \#10 metal substrate fragment. Collected in positive mode.

Figure 49. Full scan high-resolution mass spectrum depicting identification of automotive grease from post-blast residues extracted with hexane from IED \#10 plastic substrate fragment. Collected in positive mode.

Figure 50. Full scan high-resolution mass spectrum depicting identification of automotive grease from post-blast residues extracted with hexane from IED \#10 rubber substrate fragment. Collected in positive mode.

Figure 51. Negative mode, full scan high resolution mass spectrum upon operation of the DARTMS, depicting the endogenous ions. Total ion count $10^{6}$. .86

Figure 52. Analysis of water deposited onto a QuickStrip, in negative mode using full scan. Total ion count $10^{6}$.

Figure 53. Negative mode, full scan high-resolution mass spectrum for ammonium nitrate analyzed as a reference material.

Figure 54. Negative mode, full scan high-resolution mass spectrum for a commercially available stump remover (commercial source of $\mathrm{KNO}_{3}$ ), analyzed as a reference material. 88

Figure 55. Identification of $\mathrm{KNO}_{3}$ in negative mode via full scan high resolution DART-MS analysis of post-blast residues extracted from metal substrate fragments from IED \#10 with water.

Figure 56. Identification of $\mathrm{KNO}_{3}$ in negative mode via high-resolution DART-MS analysis of postblast residues extracted from plastic substrate fragments from IED \#10 with water.

Figure 57. Identification of $\mathrm{KNO}_{3}$ in negative mode via full scan high-resolution DART-MS analysis of post-blast residues extracted from rubber substrate fragments from IED \#10 with water.

Figure 58. Determination of ions characteristic of the thermal breakdown products.

Figure 59. Relative solubility of residues containing thermal breakdown products. 94

Figure 60. Positive mode, full scan: high-resolution mass spectra for diesel, analyzed as a reference material......

Figure 61. Positive mode, full scan: high-resolution mass spectra for lamp oil, analyzed as a reference material. 102 
Figure 62. Positive mode, full scan: high-resolution mass spectra for Vaseline, analyzed as a reference material.

Figure 63. Positive mode, full scan: high-resolution mass spectra for wax, analyzed as a reference material.. 


\section{List of Schemes}

Scheme 1. Reaction scheme for the electronic or vibronic production of metastable species $\left(\mathrm{M}^{*}\right)$ from inert gas (M) occurring in the source via a series of electrodes.

Scheme 2. Reaction schemes to produce secondary ionizing species (ionized water clusters) in positive mode via reaction of metastable species produced by the DART source with atmospheric reagents.

Scheme 3. Reaction schemes to produce ionized analyte species $\left(\mathrm{S}^{+\bullet}\right)$ in positive mode via reaction of secondary ionized species and analyte molecules presented to the source region.

Scheme 4. Reaction schemes for production of negative ionized analyte species (S-) via 


\section{List of Abbreviations}

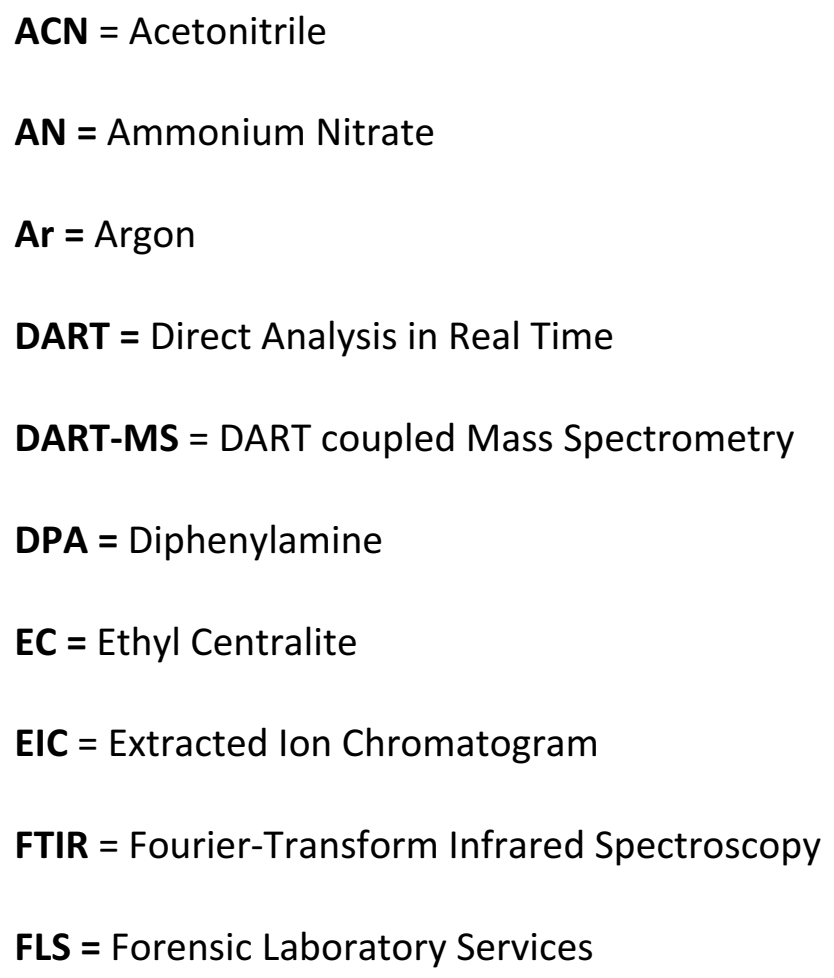




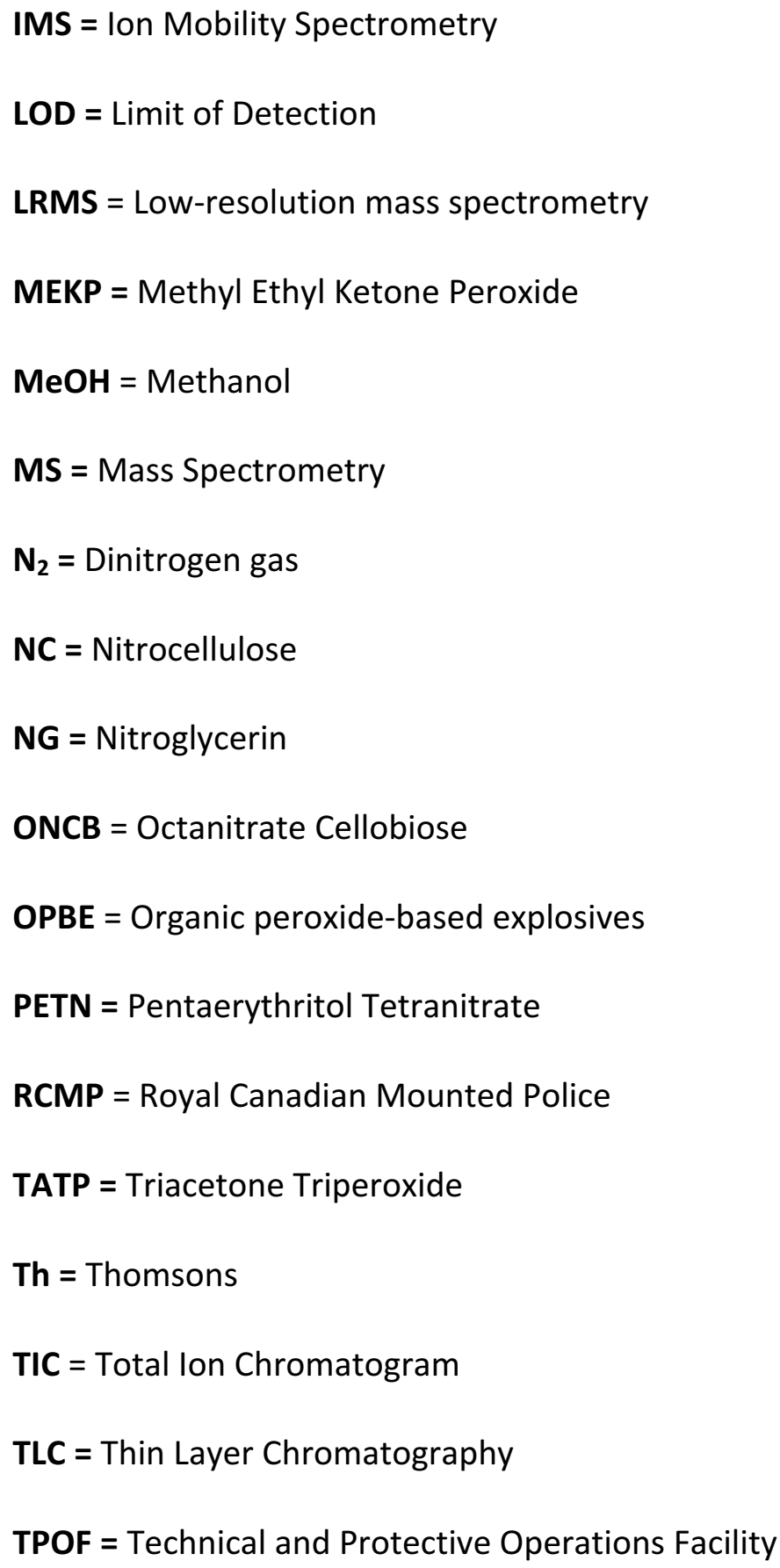




\section{Foreword}

Forensic science is the application of science to law and is a subject of great fascination to the public at large as often portrayed in mainstream entertainment media and Hollywood cinematographic movies. ${ }^{1}$ Entertainment media for public consumption often portrays a distorted or embellished view of activities involved with forensic science. The truth about advantages and limitations, policies and regulations are often misrepresented. In Canada, forensic science services are delivered by scientists who work at all levels of government, including federal, provincial and municipal. Medical coroners are either municipally or provincially regulated. Forensic analysis services requested by municipal and provincial police agencies are funded by the provincial government in Ontario and Québec and are performed by scientists at the Centre of Forensic Science in Toronto for Ontario or the Laboratoire de sciences judiciaires et de médecine légale du Québec in Québec, respectively. ${ }^{1}$ The Royal Canadian Mounted Police (RCMP) Forensic Laboratory Services (FLS) delivers forensic analysis services to all police agencies outside of Québec and Ontario, and for all federal policing activities in all provinces across the country. $^{1}$

Many streams of science (e.g. biology, psychology, toxicology and chemistry) are utilized considerably to assist in criminal investigations. ${ }^{2}$ Locard's exchange principle states that when two objects come into contact there is always a transfer of material between them, even if only at the microscopic level. The consequence of Locard's exchange principle in forensic science is that any action made by an individual in commission of a crime leads to the production of evidence. The power of such evidence 
lies in the ability to detect, identify and understand the information provided for capturing the individual or re-creating the circumstances of the crime committed. ${ }^{2}$ Chemical analyses provides police with answers to investigational questions to ensure valid identification and source attribution of evidence found the scene of a crime. Explosive materials represent a serious hazard as they can be illicitly used for mass destruction and injury or death. Detection and identification of explosives thus remain critical to ensure public safety, infrastructure security, and bolster counter-terrorism readiness. After an explosion, recovering and analyzing bomb fragments can provide important forensic links for the ensuing investigation, especially in cases where there are no biological traces (i.e. DNA) found at the scene. The substrate, size, and degree of burning of the fragments collected post-blast can provide necessary information for the re-construction of the explosive device. Post-blast residues will yield unconsumed and combustion products ${ }^{3}$ from the original energetic material, which provides the necessary information to identify the type and the source of the explosive filler used in the device.

Various field and laboratory techniques are available for detection of explosives, both pre- and post-blast. ${ }^{4}$ Ion mobility spectrometry (IMS) remains a popular technique for rapid field detection of a wide variety of explosives and is extensively used for prescreening people and objects at security checkpoints as they are easily programmable making them user friendly for front line staff. ${ }^{5}$ Matrix interference arising from environmental contaminants or other congeners can affect the discriminating power of IMS and test results are largely used for presumptive purposes only. ${ }^{6,7}$ Furthermore, the complexity of post-blast samples necessitates a multiplexed analytical scheme to 
uniquely characterize the various organic and inorganic components of explosive residues apart from matrix interference. ${ }^{8}$ Therefore, much attention is devoted to chromatography and mass spectrometry ${ }^{9,10}$ because the requisite sensitivity and selectivity is well established and accepted ${ }^{8}$ in courts of law. However, these methods remain stationary, laboratory based, and wait times for results can be lengthy if laborious sample processing is required; all of which can result in frustrating delays at the early stages of an investigation.

Recent research on explosive detection has begun to focus on ambient mass spectrometry (MS) as it provides mechanisms for rapid detection and identification that does not require complex sample preparation. ${ }^{11}$ Primary technologies providing ambient MS capabilities include desorption electrospray ionization (DESI) and direct analysis in real time (DART); both ambient ionization sources were developed in the early 2000s. ${ }^{11}$ By eliminating the constraints suffered by common ionization sources such as electrospray ionization (ESI) and matrix assisted laser desorption/ionization (MALDI), ambient ionization sources provide capabilities to analyze sample surfaces directly instituting rapid and high-throughput sampling regimes. Inorganic and organic explosive materials have both been identified and quantified using ambient ionization techniques. $12,13,14$ However, literature lacks in proving the applicability of these techniques to be able to identify explosive materials from genuine post-blast fragments. 


\section{Introduction}

\subsection{Explosives}

Explosives are energetic materials that upon ignition undergo rapid exothermic decomposition to instantaneously release high pressure gas, heat and light. ${ }^{15}$ Decomposition is predominately driven towards the production of more thermodynamically stable products, namely $\mathrm{CO}_{2}, \mathrm{~N}_{2}$ and $\mathrm{H}_{2} \mathrm{O}$. The kinetic stability of an explosive is affected by conventional reactivity trends based on structure and bond strength. The weak oxygen-oxygen bond in peroxide explosives and the nitrate specific carbon-oxygen bond in nitrated organic explosives increases reactivity leading to production of kinetically stable products. ${ }^{16}$ In the case of peroxides, instability of the peroxide bond (-O-O-) is attributed in part to the electron repulsion between the two electron-rich oxygen atoms. ${ }^{16}$ The degree of kinetic stability of any explosive will largely determine ignition sensitivity.

The mechanism of an explosion is in fact a rapid combustion reaction, wherein the oxidizer decomposes to supply oxygen to support combustion of the fuel. If the mixture is sufficiently sensitive to shock, however, it will detonate instead of simply burn. ${ }^{17} \mathrm{High}$ order explosives detonate creating a supersonic explosive shock front that travels at velocities greater than $1000 \mathrm{~m} \cdot \mathrm{s}^{-1} .{ }^{18}$ Low order explosives deflagrate by surface burning that occurs at speeds less than $1000 \mathrm{~m} \cdot \mathrm{s}^{-1} .{ }^{18}$ Explosives extremely sensitive to energetic stimuli are classified as primary explosives; often used as an initiator for larger less sensitive main charges. Secondary explosives are relatively less sensitive to shock; often used as the main charge as they are safer to handle, transport and store. ${ }^{18}$ Tertiary 
explosives are relatively insensitive requiring initiation by larger amounts of primary and/or secondary explosives, such as so-called boosters. ${ }^{18}$

To date, the number and varieties of different explosive materials has grown immensely along with many different applications. Different types of explosives can be categorized using a variety of classification schemes, depending on the property or measurement of interest, including use, chemical composition, energetics, or blast properties. Explosives of significant concern are those accessible for use in criminal or terrorist activity. In a Canadian context, commercially-available firearm propellants and consumer fireworks are commonly used as explosive fillers for IEDs, but homemade explosives (HME) are an ever-increasing threat due to ease of fabrication using reagents sourced from common household chemicals. HME include any explosive material that has been altered beyond its intended use, has been created by combining products together or has been synthesized from readily available reagents. ${ }^{19}$

After a bombing, rapid source attribution of the explosive filler used as having been either a commercial or HME product can provide key forensic links for the ensuing investigation. Equally as important, early identification of explosive traces can provide pivotal investigative forensic intelligence to help prevent a tragedy from occurring. Therefore, delivering quality assured answers to front-line personnel with faster turnaround times motivates improvement of the methods used for explosive trace detection. Many different analytical schemes have been developed to achieve this goal. 


\subsubsection{Homemade explosives}

\subsubsection{Peroxide Explosives}

Organic peroxides are highly reactive compounds containing oxygen-oxygen bonds. The electron repulsion experienced by the lone pair of both oxygen atoms in the peroxide bond decreases the energy required to break the bond. ${ }^{20}$ Organic peroxidebased explosives (OPBE) require no confinement to detonate and produce high-pressure shock waves traveling at speeds between $4500-5500 \mathrm{~m} \cdot \mathrm{s}^{-1}$, classifying them as high-order explosives. ${ }^{21}$ With respect to sensitivity, OPBE are classified as primary explosives due to extreme sensitivity to any energetic stimuli. ${ }^{21}$ These materials pose significant concern as the synthesis uses readily available and common household products requiring very basic knowledge or training. ${ }^{22}$ Upon synthesis and use as explosive filler in an IED, OPBEs cause considerable damage and harm, as unfortunately demonstrated by several domestic and international terrorist attacks in recent years. ${ }^{23,24}$ The detection and identification of OPBEs remains a critical operation in ensuring public safety.

Triacetone triperoxide (TATP), hexamethylene triperoxide (HMTD) and methyl ethyl ketone peroxide (MEKP) OPBE are the most common clandestine OPBEs encountered in criminal investigations and forensic laboratories (Figure 1-3). TATP and HMTD are very sensitive to impact, heat and friction and find no legitimate use as commercial or industrial manufactured explosives. ${ }^{25}$ MEKP is slightly less sensitive and has found use in dilute solutions as a polymerization catalyst in commercial manufacture of polyester and acrylic resins. ${ }^{26}$ 
The reagents required to synthesize OPBEs are commercially available and synthetic methods are available from many illicit internet sources, such as chatrooms or other forms of social media. ${ }^{22}$ Synthesis of OPBE occurs via step-wise insertion mechanistic steps. ${ }^{26}$ If an insertion step produces a stable product the final yield may contain mixtures of linear or cyclic dimer, trimer and tetramer forms; observed in the synthesis of TATP and MEKP. ${ }^{26}$ The trimer acetone peroxide (i.e. TATP) is produced as the most abundant product, compared to a mixture of oligomers produced in the synthesis of MEKP. ${ }^{26}$ Purification of these synthetic products requires difficult and resourceintensive methods resulting in the use of impure products in IEDs. The homemade nature, limited solubility, lack of UV absorbance or fluorescence moieties and sensitivity to mechanical stress creates many analytical challenges for the detection and identification of OBPE.

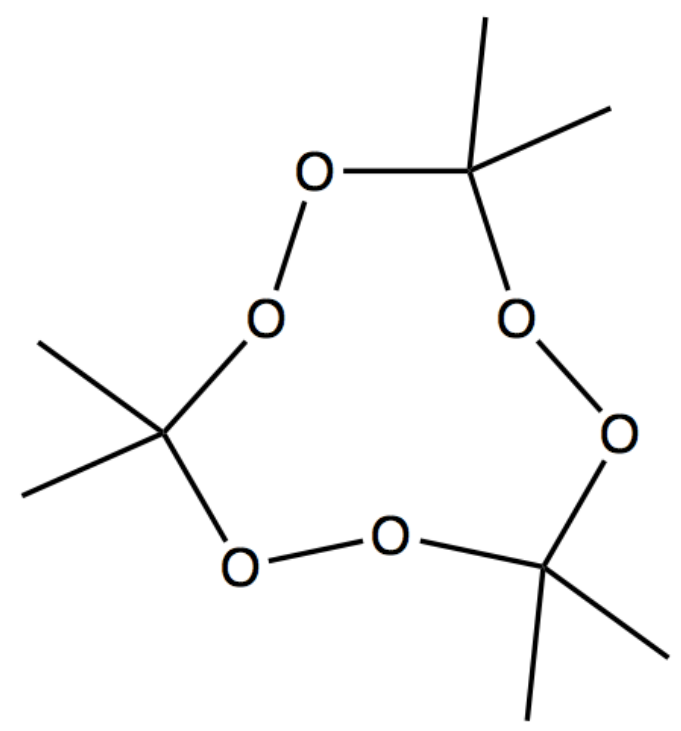

Figure 1. Triacetone triperoxide (TATP). 


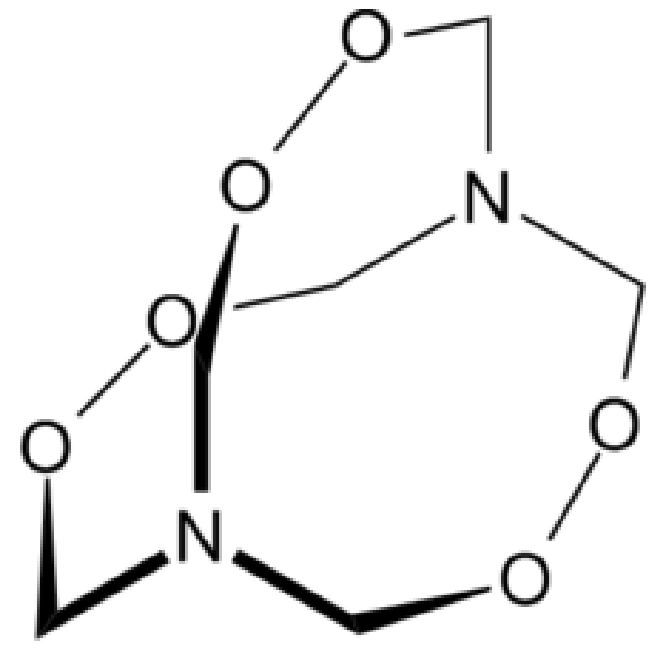

Figure 2. Hexamethylene triperoxide (HMTD).

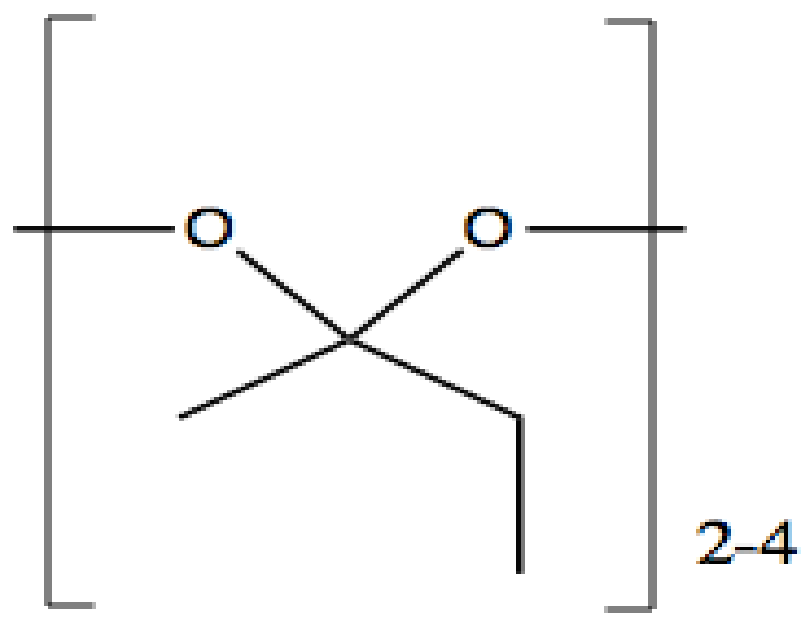

Figure 3. Methyl ethyl ketone (MEKP). 


\subsubsection{Binary Explosives}

Binary explosives are mixtures consisting of two components blended together, namely: a combustible fuel and strong oxidizer. Separately, neither component is explosive, but upon mixing together in the correct ratio the resulting blend will behave as an explosive upon shock with sufficient force. Upon ignition of binary explosives highpressure shock waves traveling at speeds between $2500-4500 \mathrm{~m} \cdot \mathrm{s}^{-1}$ are produced classifying them as high-order explosives. ${ }^{27}$ With respect to sensitivity, most binary explosives are classified as tertiary explosives as they require significant energy for initiation and are safe to handle, transport and store. Most commercially manufactured explosives intended for use in mining, quarrying and blasting are a based upon a binary explosive formulation, albeit other different additives are regularly included (e.g. emulsifiers, plasticizers, binders, etc). ${ }^{27}$ Stringent regulatory requirements stipulate that commercial explosives must be secured in licensed magazines to limit unauthorized access and prevent theft. Consequently, clandestine fabrication of homemade binary explosive mixtures has become an attractive alternative for criminal activity, because the oxidizer and fuel components can be sourced from commercial household products. Many petroleum-based products can be used as the fuel source. Sugar-based food products, such as starch or confectionary sugar, can also be used as a suitable combustible fuel source. Commercial fertilizers, compression-type instant cold packs and stump removers are all sources of suitable strong oxidizers. By simply mixing or blending the correct combination of combustible fuel with a strong oxidizer a binary explosive can be prepared. 
Table 1. Examples of products that source components for binary explosives.

\begin{tabular}{|c|c|c|c|}
\hline \multicolumn{2}{|c|}{ Fuel Precursors } & \multicolumn{2}{|c|}{ Oxidizer Precursors } \\
\hline Component & Source & Component & Source \\
\hline \multirow[b]{2}{*}{ Petroleum based } & \multirow{2}{*}{$\begin{array}{c}\text { Diesel } \\
\text { Automotive } \\
\text { Grease } \\
\text { Paraffin Wax } \\
\text { Lamp Oil } \\
\text { Vaseline }\end{array}$} & Potassium Chlorate & $\begin{array}{c}\text { Textiles, matches, } \\
\text { pyrotechnics }\end{array}$ \\
\hline & & $\begin{array}{l}\text { Potassium } \\
\text { Perchlorate }\end{array}$ & $\begin{array}{c}\text { Airbag initiator, } \\
\text { pyrotechnics }\end{array}$ \\
\hline \multirow[t]{2}{*}{$\begin{array}{c}\text { Carbohydrate } \\
\text { based }\end{array}$} & \multirow{2}{*}{$\begin{array}{l}\text { Starch } \\
\text { Sucrose } \\
\text { Flour }\end{array}$} & Ammonium Nitrate & $\begin{array}{c}\text { Fertilizers, } \\
\text { Cold packs, } \\
\text { Exploding targets }\end{array}$ \\
\hline & & Potassium Nitrate & Stump Remover \\
\hline
\end{tabular}

Current accredited methods utilized by the RCMP for detection and identification of binary explosives from recovered from post-blast residues include gas chromatography-mass spectrometry (GCMS), ion chromatography-mass spectrometry (ICMS) and Fourier-transfer infrared spectroscopy (FTIR). Chromatography methods combined with mass spectrometry are well suited to separate the different components of binary explosives and identify each component in isolation. The specific choice of which technique is used will depend on the chemical composition and phase of the actual binary explosive examined (e.g. ICMS for water-soluble salts, GCMS for volatile organics). With the identification of both components, the overall energetic mixture can be exposed. 


\subsubsection{Smokeless Powder Products}

Modern smokeless powders are the propellants used in small firearm ammunition.

${ }^{28}$ Ignition of the propellant the energy released, via formation of gaseous products, results in the ejection of the bullet from the chamber of a gun. ${ }^{28}$ Smokeless powders are largely produced and used in the assembly of self-packed ammunition. However, smokeless powder can also be procured for illicit use in IEDs. ${ }^{28,29}$ These products produce negligible smoke when ignited and burned as they are largely composed of organic explosives that produce only $\mathrm{CO}_{2}$ and $\mathrm{H}_{2} \mathrm{O}$ gaseous products upon combustion. In contrast, other propellants, such as black powder, mainly produce solid, non-volatile products upon combustion thus producing airborne black soot visible as smoke.

Nitrocellulose (NC) is the base organic explosive used to manufacture smokeless powders (Figure 4). However, nitroglycerine (NG) can also be used in certain types of smokeless powders to increase the output energy (Figure 5). Single-base smokeless powder contains NC only and double-base products containing NC and NG. A double-base smokeless powder may contain between $10-50 \%$ NG content by weight depending on the product used. Because NG can detonate it has the potential to shatter a firearm. NC is obtained from nitration of cellulose; nitration is an exothermic esterification reaction whereby various pendant hydroxyl groups are nitrated but the $\beta$ (1-4) linkages between monomer units in the cellulose chain are not broken. ${ }^{30}$ Each glucose monomer contains three potential hydroxyl groups that can be nitrated. The degree of total nitration depends on the cellulose source and the reaction conditions. ${ }^{30} \mathrm{NC}$ can be nitrated to different, varying degrees and the final extent of nitration can affect the commercial use of the NC prepared. Highly-nitrated NC is considered to have a nitrogen content of at least 
$14 \%$ by weight and is the form used in smokeless powders. ${ }^{30}$ Lesser nitrated NC is used to fabricate cigarette paper and party streamers. Stabilizers, plasticizers and surface coatings are used in different smokeless powders to modify or improve performance characteristics and prolong shelf life of the final propellant powder. A common example is diphenylamine (DPA) which is a weak base added to smokeless powders to neutralize the slightly, naturally acidic NC and prevent spontaneous decomposition over time (Figure 6). ${ }^{28,29}$ Another common example is ethyl centralite (EC) which is a both a plasticizer and flame retardant to raise ignition temperature and slow the burning rate of the propellant powder (Figure 7). ${ }^{28,29}$

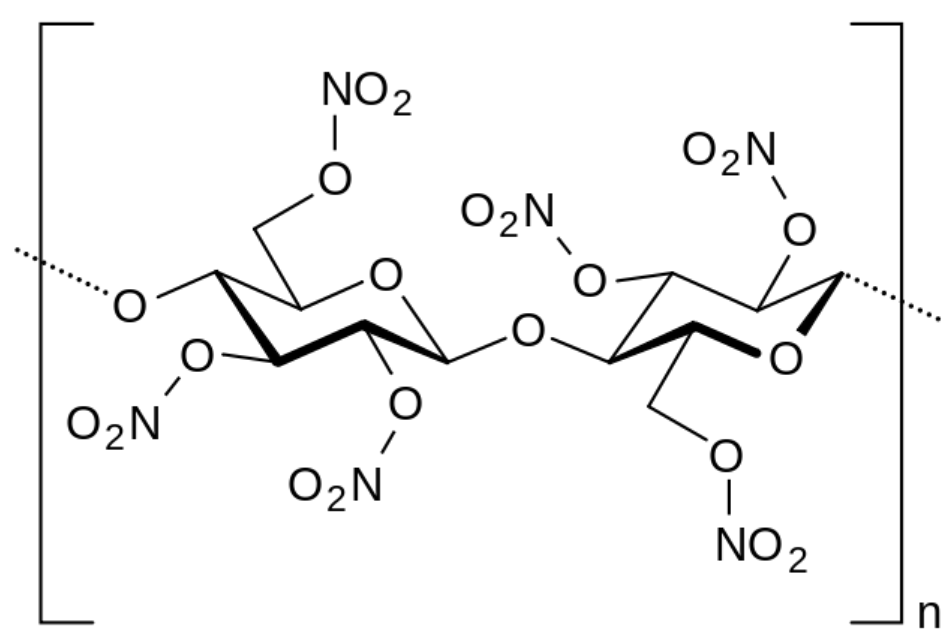

Figure 4. Fully nitrated nitrocellulose. 


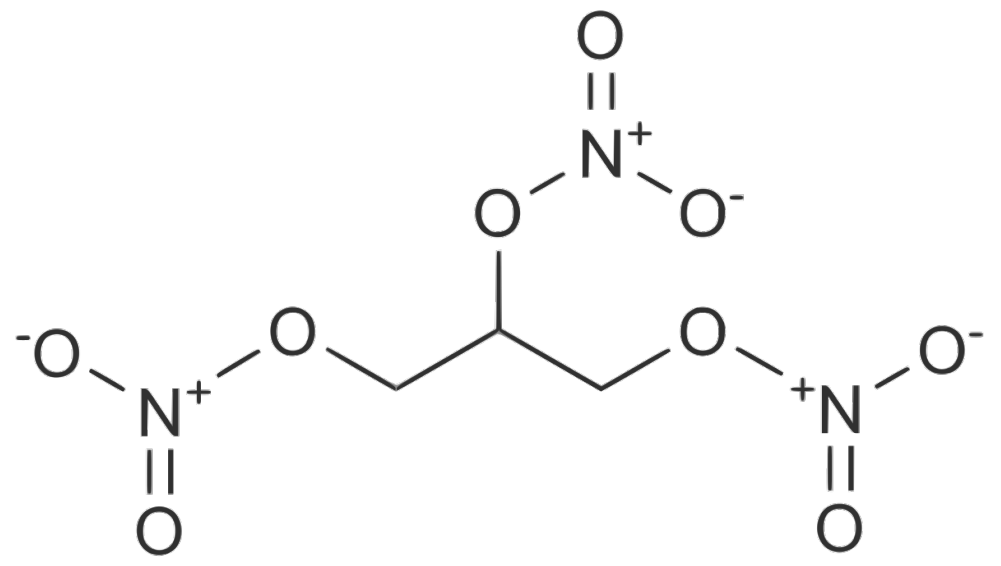

Figure 5. Nitroglycerin.

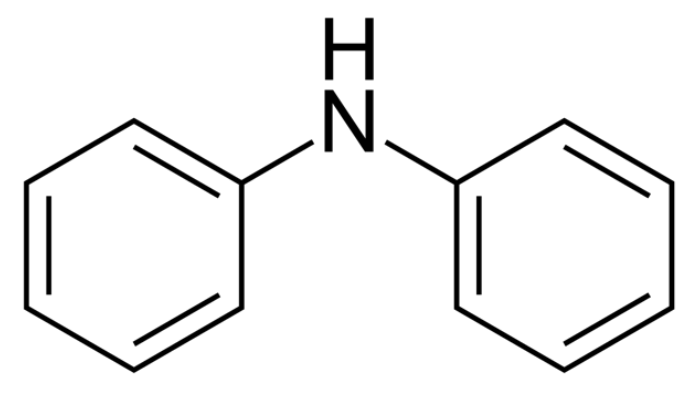

Figure 6. Diphenylamine.

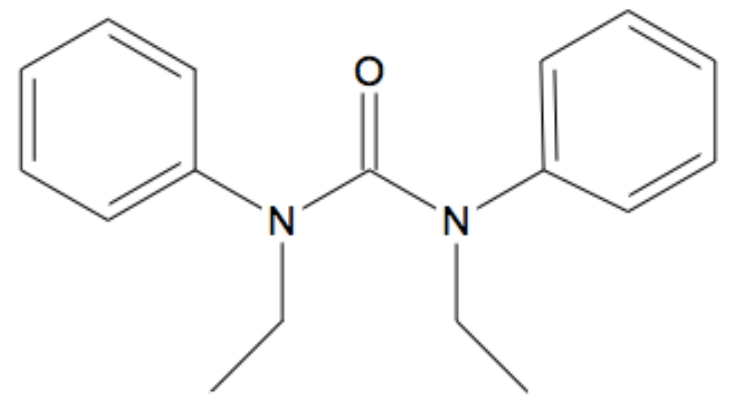

Figure 7. Ethyl centralite 
Methods to characterize NG, NC and the various additives from smokeless powders have been developed. ${ }^{31-35}$ However, current methods to identify nitrocellulose rely upon recovery of an intact propellant grain from which the various constituents (NC, NG, additives) can be extracted and characterized. No method has been previously reported to identify nitrocellulose post blast based on its thermal-degradation products in the absence of a recoverable intact grain for analysis. Identification of the thermal breakdown products of nitrocellulose remains challenging due to absence of a characteristic reference material. 


\subsection{Explosive Analysis}

\subsubsection{Ion Mobility Spectrometry (IMS)}

Ion mobility spectrometry (IMS) is a commonly used technique to screen for contraband at border security checkpoints, including concealed drugs and explosives. Detection of illicit materials occurs by observing characteristic mobility of ions converted from sample vapors in a weak electric field. ${ }^{36}$ Most IMS instrumentation is engineered to include four main sub-components: an ion source, an ion gate, a drift tube and a detector (Figure 8). ${ }^{36}$ Upon ionization of sample vapors in the source, the ion gate electronically ejects ions into the drift tube whereby an electric field is applied. ${ }^{12}$ As ions experience the electric field they move towards the detector, which in most devices is a Faraday cup. ${ }^{36}$ Upon collisional neutralization at the detector, current flow is collected as a measurable signal. ${ }^{36} \mathrm{~A}$ mobility spectrum is produced, plotting ion current against drift time. Based solely on a specimen's drift time, detection and identification of illicit materials is achievable.

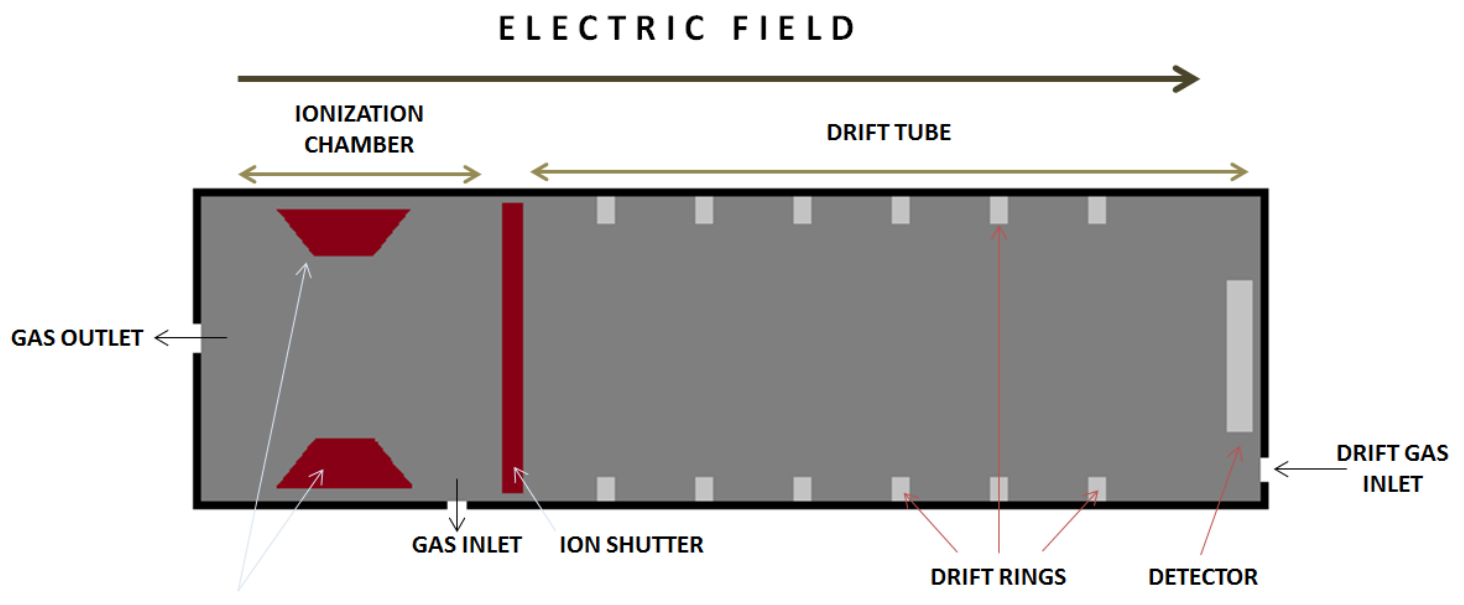

IONIZATIONSOURCE

Figure 8. Schematic diagram of an ion mobility spectrometer (IMS). 
Ion mobility is one preferred method used to screen for explosive residues as it is relatively inexpensive, easy to use and provides capability for rapid analysis that is field deployable, all while maintaining high sensitivity. ${ }^{36}$ However, due to single mechanism discrimination (i.e. drift time) loss of sensitivity and selectivity due to matrix interference arising from environmental contaminants and other congeners remains a significant critique of IMS technology. Therefore, with respect to identification and detection these methods largely remain presumptive tests. Due to the complexity of post-blast samples multiplexed analytical schemes are required to uniquely characterize the various organic and inorganic components of explosive residues apart from matrix interference. ${ }^{37}$

\subsubsection{Chromatography Methods}

Many forensic laboratories are equipped with severeal chromatographic instrumentation as they remain the gold-standard techniques for separation, identification and quantification of compounds in a mixture. ${ }^{38}$ All chromatography methods include a mobile and stationary phase. Physical separation of a mixture is based on different partitioning factors of components in the mixture between the mobile and stationary phases. ${ }^{38}$ Factors such as adsorption, affinity, polarity and size affect separation processes. ${ }^{38}$ Many different detectors are coupled to chromatography instrumentation providing identification mechanisms based on structure, mass, charge, volatility and polarity. Separation techniques are included in many forensic practices because forensically-relevant samples often contain many unknown compounds in a complex mixture. Inclusion of chromatography methods provide sensitive and selective detection, identification and quantification of forensically relevant compounds, such as 
explosives. Therefore, much attention has been invested in using chromatography methods for explosive analysis. ${ }^{9,10}$

High-performance liquid chromatography (HPLC), gas-chromatography (GC) and ion chromatography (IC) are the most common chromatography methods utilized for explosive analysis. ${ }^{39}$ HPLC employs a closed, pressurized column containing a solid phase (Figure 9). At high-pressures the mobile phase is passed through a column carrying components to be separated. ${ }^{38}$ Separation occurs via differences in analyte relative affinity for the mobile and stationary phases. The polarity of the analytes dictates relative affinity for mobile and stationary phases providing a mechanism for physical separation of the components. ${ }^{38}$ Sample mixtures are initially loaded onto the column via affinity for the stationary phase. ${ }^{38}$ Upon a switch in polarity of the mobile phase, analytes are driven back to the mobile phase and elute from the column. ${ }^{38} \mathrm{HPLC}$ is a desirable separation and identification technique for explosive analysis due to its well-respected accuracy, efficiency and reproducibility. Methods can be set up in a highly automated fashion allowing for high-throughput analyses of a wide variety of samples.

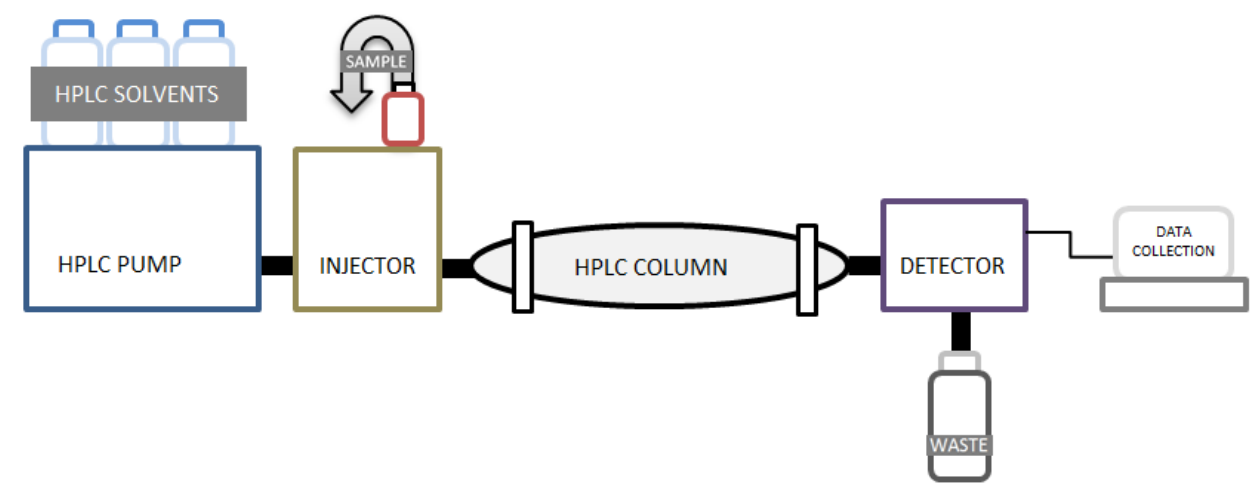

Figure 9. Schematic diagram of the instrumentation used to separate analytes of a mixture via high-performance liquid chromatography (HPLC). 
Methods involving GC are used in explosive analysis for detection and identification of volatile compounds. GC columns contain a liquid stationary phase adsorbed onto an inert solid. The mobile phase is usually composed of inert carrier gases (e.g. helium or nitrogen gas). Volatile analytes enter the gaseous mobile phase and as they pass through the column, depending on relative affinity for the mobile phase, separation occurs (Figure 10). ${ }^{14}$ As a simple, multi-faceted, rapid and highly sensitive method, GC has proven its ruggedness and robustness while providing appreciable sensitivity and selectivity required for explosive analysis. ${ }^{33-35}$

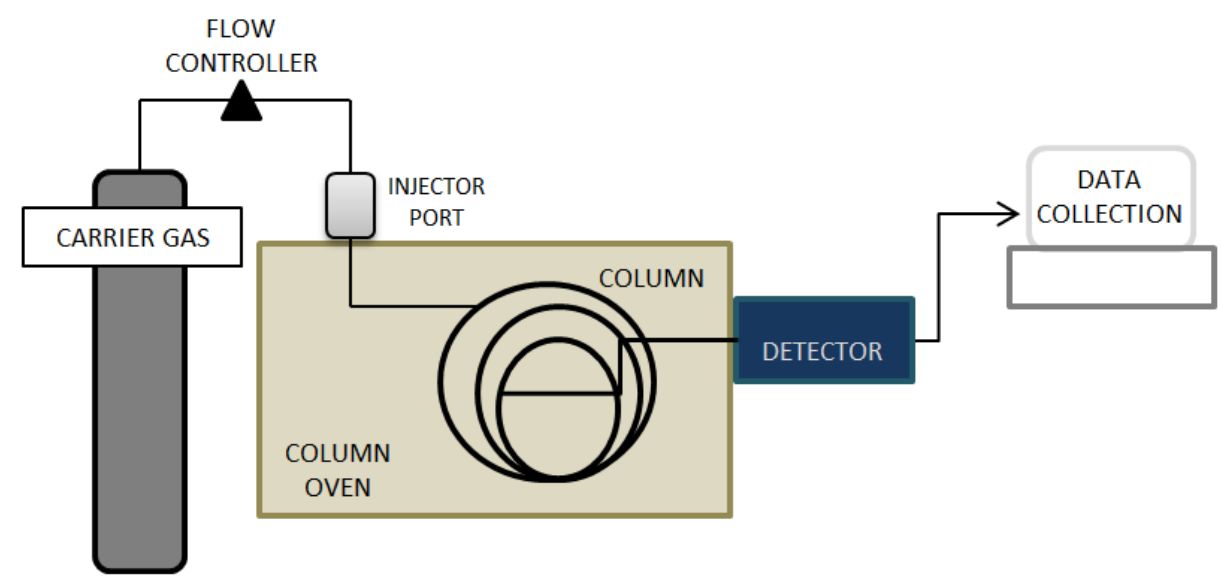

Figure 10. Schematic diagram of the instrumentation used to separate volatile components in a mixture via gas chromatography (GC). 
Due to the wide variety of explosive materials posing significant threats to our safety and security, capabilities of our analytical methods to detect and identify them continue to be challenged. ${ }^{11}$ While many explosives are organic compounds amenable to HPLC and GC methods, inorganic explosive classes are not compatible. Ion chromatography is the preferred separation technique for many explosive-related ionic species such as ionic salt oxidizers used in binary explosive mixtures (Figure 11). ${ }^{40,41}$ Based on electrostatic interactions between mobile and stationary phases, separation of ionic species occurs. Changes in $\mathrm{pH}$, concentration of ion salts and ionic strength of the buffered mobile phase are used to elute ions from the column. ${ }^{38}$ Both anion-exchange and cation-exchange columns are available. IC is a commonly used, quality assured method for analysis of inorganic explosives.

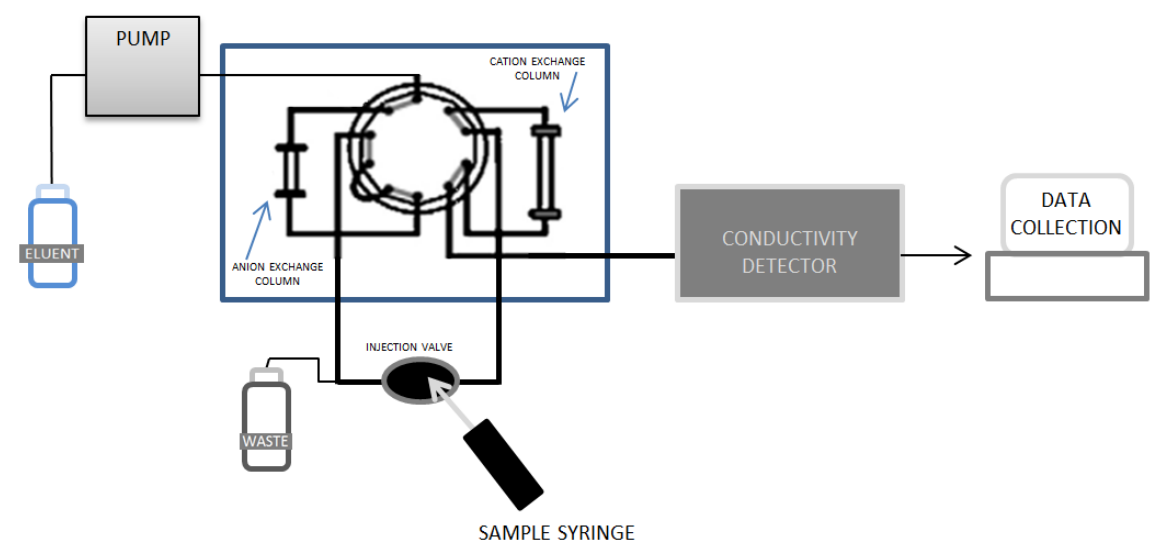

Figure 11. Schematic diagram of the instrumentation used for simultaneous separation of anions and cations in solution via ion chromatography (IC). 
Chromatography methods remain primary detection and identification methods for explosive analysis. However, the stationary and laboratory based design and required laborious sample preparation creates lengthy wait times for results. Associated frustrating delays caused at early stages of an investigation, due to limitations associated with chromatography methods, motivates adaptation, innovation and validation of new methods and techniques. Ambient-ionization mass spectrometry methods, such as DARTMS, have become lucrative methods for detection and identification of explosives as they are simple, facile and rapid methods that still maintain robustness, reproducibility, sensitivity and selectivity required for court. ${ }^{11,42-47}$ Furthermore, due to compatibility with low-resolution mobile mass spectrometers there is potential for mobility from the lab to the crime scene. ${ }^{11}$ The applicability of DART-MS for detection of many different nitro, nitrosamine and nitroaromatic explosives has been studied excessively due to prolific use of these explosives in IED. ${ }^{48}$ However, adaptability to combat the more contemporary forensic challenges associated with detection of homemade explosive has yet to be explored. 


\subsubsection{Mass Spectrometry (MS)}

Since invention in the early 1900s, mass spectrometry (MS) remains one of the most powerful analytical tools available. Predominantly used for study and recognition of matter by filtering substances based on mass-to-charge ratio $(m / z){ }^{49}$ Versatile applications of mass spectrometers results in inclusion of these instruments in laboratories of many scientific disciplines all around the world. ${ }^{50}$ With continuous innovation, adaptation, and modifications MS methods continue to prove robustness, sensitivity and selectivity.

The fundamental concepts and engineering of mass spectrometers has remained constant since invention. As described, characterization of an analyte via MS is accomplished by ionization, filtration and detection of gaseous analyte species based on the $\mathrm{m} / \mathrm{z}^{50}$ Instrumental configurations of mass spectrometers include four main components: vacuum systems, an ionization source, a mass filter and a detector. Ions produced in the source are accelerated into an electric field whereby separation based on $m / z$ takes place (i.e. mass filter). Compounds with slightly different masses result in variable $m / z$ and unique trajectories through the mass filter providing a robust mechanism for differentiation. As ions reach the detector the electrical response is plotted against $\mathrm{m} / \mathrm{z}$ to create a mass spectrum exposing identity and abundance of species present in the sample. ${ }^{17}$ Vacuum systems are incorporated to reduce likelihood and frequency of ion collisions resulting in potential charge transfers ultimately hindering the path of ions from source to detector. ${ }^{50}$ 
Compatibility of an analyte with MS methods depends on its capability to transfer to the gaseous phase and become ionized. To facilitate this required transformation, many ionization sources have been developed. Innovation has led to many modifications and adaptations producing a long list of ionization sources with compatibility to a wide variety of analytes such as: small molecules, inorganic compounds, large organic compounds and biomolecules. Ionization sources can produce ions with negative or positive charges. Historically, ionization sources have been located inside the mass spectrometer under vacuum but to date many ambient ionization sources have become available. ${ }^{51}$ Ionization sources are predominantly classified by vacuum requirements, but also are classified by the strength of the ionization. Hard-ionization sources describe ionization of a molecule by producing fragment ions from parent analyte. Soft-ionization mechanisms predominantly produce ionized parent ions with little to no production of fragment ions.

Production of ions in the source migrate through the mass filter to the detector. Many filters are available and in general, manipulate an established electromagnetic field to control the successful trajectory of an ion from the source to the detector. Mass filters are differentiated by shape, size, and material of the components; ultimately leading to differences in resolution and mass limits. Low-resolution mass spectrometers (LRMS) measure $\mathrm{m} / \mathrm{z}$ by whole number masses of atoms; high-resolution (HRMS) instrumentation provides superior mass accuracy by measuring the exact mass of each atom to the thousandth decimal place. ${ }^{51}$ HRMS instrumentation becomes extremely useful when 
masses of many analytes are similar as it has the power to resolve and uniquely characterize similar mass compounds. ${ }^{51}$

Detection of ions occurs by converting the electrical response, created when an ion reaches the detector, into representable and readable signals. Many different detectors are on the market today. Examples of commonly used detectors include photoplates, photomultipler tubes, Faraday detectors, electron-multipliers and imagecurrent detectors.

In most cases, post-blast explosive analysis relies solely on detection and identification of residues from any unconsumed explosives found post-blast. However, the destructive nature of an explosion (heat, pressure, oxidation and pyrolysis) creates considerable challenges as any undetonated material typically occurs in small amounts and is spread over many fragments across large areas of land. Therefore, quality assured methods and techniques are required for success. MS remains one of the most predominant methods used for explosive analysis from trace quantities of residues collected post-blast. 


\subsubsection{Direct Anallysis in Real Time (DART) Ionization}

DART, an ambient soft-ionization technique, was designed and engineered by Cody et al. in 2005. ${ }^{50}$ After a decade from initial release, DART sources have found their way into many food, environmental, health and industry related laboratories. DART has found specific application in food analysis, chemical identification and characterization, pesticide development and detection, drug development and screening and forensics. ${ }^{42-}$ 47, 54-55 Particularly with respect to forensic analysis, DART-MS has proven to be a powerfully reliable technique for explosive analysis. ${ }^{42-47}$

Operating in ambient environments without sample preparation requirements, DART provides capability to ionize liquid, solid and gaseous samples in their native form. ${ }^{49}$ Ionization of analyte molecules thermally-desorbed from sample surfaces occurs via production and resulting reactions with metastable species produced by the source. Heated inert gases such as helium $(\mathrm{He})$, argon $(\mathrm{Ar})$ or dinitrogen gas $\left(\mathrm{N}_{2}\right)$ enter the source and pass through a series of electrodes producing metastable species such as excited state helium (He*) (Scheme 1). Upon exiting the source, a dry stream of excited gas passes through a final electrode directing ions to the MS, removing any charged molecules to prevent undesired ion recombination and controlling the polarity mode (i.e. positive vs. negative mode) (Figure 12). ${ }^{53}$

$$
M+\text { energy }=M^{*}
$$

Scheme 1. Reaction scheme for the electronic or vibronic production of metastable species $\left(M^{*}\right)$ from inert gas $(M)$ occurring in the source via a series of electrodes. 


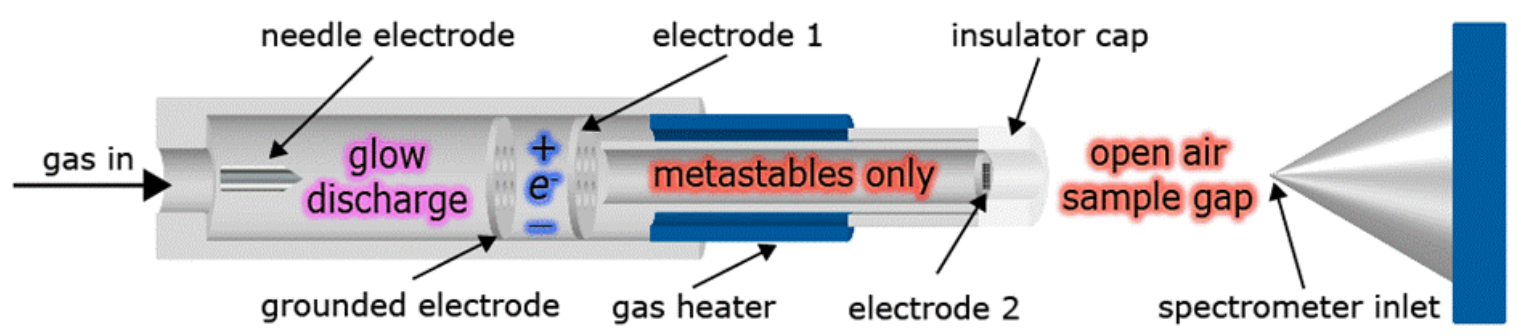

Figure 12. Schematic diagram of DART source.

Mechanisms responsible for producing ionized analyte molecules are dictated by the inert carrier gas used. $\mathrm{He}$ and $\mathrm{N}_{2}$ are the most common with the former reported to be most effective. ${ }^{53}$ Metastable species exiting the source react with atmospheric molecules to produce reagent ions. Upon reaction between $\mathrm{He}^{*}$ and atmospheric species, ionized reagent molecules are produced via Penning ionization. ${ }^{19}$ Ionized water clusters, the primary reagent ion produced, are responsible for consecutive ionization of thermally-desorbed analyte molecules. ${ }^{53}$ Applicability of this ionization source is limited to molecules with mass ranges from m/z 50-1200 as many compounds over m/z 1200 lack required volatility. ${ }^{53}$ Exact mechanisms for production of these reactive species is not clear however further investigation has led to a few proposals. ${ }^{49}$ Protonation, deprotonation, direct charge transfer and adduct ion formation are key mechanisms responsible for production of positively charged analyte molecules (Scheme 2, 3). Negatively charged analyte molecules are produced by flipping the potential on the end grid electrode to negative potentials. Electrons produced by Penning ionization undergo electron capture with atmospheric molecules in the reactive zone between the source and the MS (Scheme 4). ${ }^{49}$ 

(a) $\mathrm{M}^{*}+\mathrm{N}_{2} \rightarrow \mathrm{M}+\mathrm{N}_{2}^{+\bullet}+\mathrm{e}^{-}$
(b) $\mathrm{M}^{*}+\mathrm{H}_{2} \mathrm{O} \rightarrow \mathrm{M}+\mathrm{H}_{2} \mathrm{O}^{+\bullet}+\mathrm{e}^{-}$
(c) $\mathrm{N}_{2}^{+\bullet}+\mathrm{N}_{2}+\mathrm{N}_{2}$ (3rd body) $\rightarrow \mathrm{N}_{4}^{+\bullet}+\mathrm{N}_{2}$ (3rd body) $\mathrm{N}_{4}^{+\bullet}+\mathrm{H}_{2} \mathrm{O} \rightarrow 2 \mathrm{~N}_{2}+\mathrm{H}_{2} \mathrm{O}^{+\bullet}$
(d) $\mathrm{H}_{2} \mathrm{O}^{+\bullet}+\mathrm{H}_{2} \mathrm{O} \rightarrow \mathrm{H}_{3} \mathrm{O}^{+}+\mathrm{OH}^{\bullet}$ $\mathrm{H}_{3} \mathrm{O}^{+}+n \mathrm{H}_{2} \mathrm{O} \rightarrow\left[n \mathrm{H}_{2} \mathrm{O}+\mathrm{H}\right]^{+}$

Scheme 2. Reaction schemes to produce secondary ionizing species (ionized water clusters) in positive mode via reaction of metastable species produced by the DART source with atmospheric reagents.

$$
\begin{gathered}
\mathrm{S}+\left[n \mathrm{H}_{2} \mathrm{O}+\mathrm{H}\right]^{+} \rightarrow[\mathrm{S}+\mathrm{H}]^{+}+n \mathrm{H}_{2} \mathrm{O} \\
\mathrm{S}+\mathrm{N}_{4}^{+\bullet} \rightarrow \mathrm{S}^{+\bullet}+2 \mathrm{~N}_{2} \\
\mathrm{~S}+\mathrm{O}_{2}^{+\bullet} \rightarrow \mathrm{S}^{+\bullet}+\mathrm{O}_{2} \\
\mathrm{~S}+\mathrm{NO}^{+} \rightarrow \mathrm{S}^{+\bullet}+\mathrm{NO} \\
\mathrm{S}+\left[\mathrm{NH}_{4}\right]^{+} \rightarrow\left[\mathrm{S}+\mathrm{NH}_{4}\right]^{+}
\end{gathered}
$$

Scheme 3. Reaction schemes to produce ionized analyte species $\left(\mathrm{S}^{+\bullet}\right)$ in positive mode via reaction of secondary ionized species and analyte molecules presented to the source region. 


$$
\begin{aligned}
\mathrm{O}_{2}+\mathrm{e}^{-} & \rightarrow \mathrm{O}_{2}^{-\bullet} \\
\mathrm{S}+\mathrm{O}_{2}^{-\bullet} & \rightarrow \mathrm{S}^{-\bullet}+\mathrm{O}_{2} \\
\mathrm{~S}+\mathrm{e}^{-} & \rightarrow \mathrm{S}^{-\bullet} \\
\mathrm{SX}+\mathrm{e}^{-} & \rightarrow \mathrm{S}^{-}+\mathrm{X}^{-} \\
\mathrm{SH} & \rightarrow[\mathrm{S}]^{-}+\mathrm{H}^{+}
\end{aligned}
$$

Scheme 4. Reaction schemes for production of negative ionized analyte species (S-) via

In summary, operation in positive mode predominantly produces protonated analyte molecules and in negative mode produces deprotonated molecules. ${ }^{49}$ Depending on the analyte molecule, other ionized species may be favorable (i.e. ammonium and chloride adducts). Coupling to a mass spectrometer, analysis in either mode provides relatively simple mass spectra.

To facilitate the transition of ions from the ambient ionization reaction zone to the mass spectrometer under high vacuum, an interface housing skimmer orifice with slight potential differences between them is installed on the front end. ${ }^{53}$ These components of the interface are responsible for removing neutral contaminations and directing ionized species into the MS. A rough pump connected to the interface and is used to remove neutral contamination. 
Operation of DART-MS can occur in surface desorption mode or transmission mode simply by manipulating orientation of the source with respect to the MS-interface. Set at $45^{\circ}$ (Figure 13 (a)) surface desorption of analyte molecules occurs providing a simple, easy to use method for direct analysis. Set at $0^{\circ}$, (Figure 13 (b)) transmission analysis of samples is possible providing mechanisms for analysis of liquids or samples found on porous materials. Commercially available consumable support mechanisms are available for analysis of solid and liquid samples. By simply placing the samples onto retro fitted supports, which are set into a mechanical rail (Figure 16. (b)), a reproducible mechanism to move the samples into and out of the reaction zone between the DART and the MS is provided.
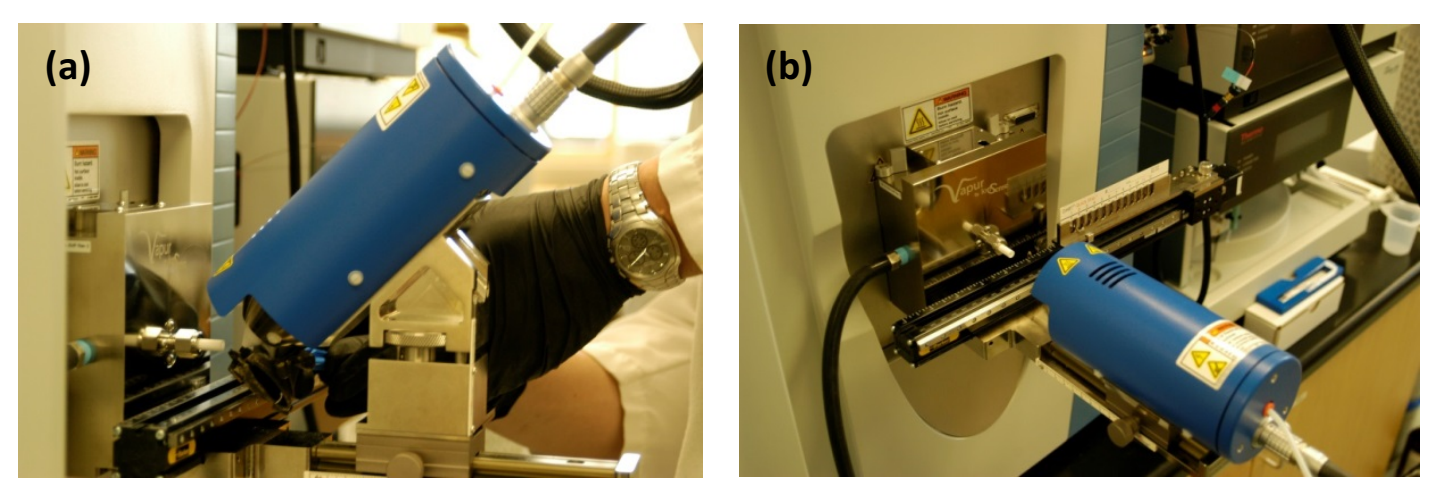

Figure 13. Images of the different orientations of the DART source with respect to the MS interface: (a) surface desorption mode and (b) transmission mode. 


\subsubsection{Q-Exactive Mass Spectrometer}

As previously described, the mass analyzer is a key component of a mass spectrometer as it provides capability to filter and selectively detect species with specific $\mathrm{m} / \mathrm{z}$. LRMS include mass analyzers such as quadrupoles and linear ion traps which can detect ions based on nominal m/z. HRMS demonstrates its power of offering accuracy, sensitivity and selectivity simply by providing $\mathrm{m} / \mathrm{z}$ measurements to the thousandth decimal place. ${ }^{52}$ Instrumental platforms available include Fourier-transform (FT) ion cyclotron resonance (ICR), time-of-flight (TOF) and Orbitrap mass analyzers (Figure 14). ${ }^{52}$ Using HRMS for explosive analysis provides capability for detection and identification of explosives from complex sample matrix with the sensitivity, selectivity, and accuracy required by the courts of law.

By combining the Orbitrap and quadrupole instrumentation, the hybrid technology coined the Q-Exactive was released in $2011 .{ }^{56}$ Since original design and engineering the Q-Exactive encompasses a quadrupole, C-trap, High-energy Collisional Dissociation (HCD) cell and the Orbitrap mass analyzer. ${ }^{56}$ Selectivity of desired ions is offered by manipulating the electromagnetic field established between the quadruples.

${ }^{57}$ Ions that do not have the specific $\mathrm{m} / \mathrm{z}$, migrate irregularly through the field, crashing into the rods or the sides of the mass analyzer and do not reach the detector. ${ }^{57}$ The Ctrap collects ions into packets prior to injection into the HCD or the Orbitrap. Within the HCD cell, fragmentation of ions occurs when an increase in the kinetic energy of the ions results in collisions with neutral molecules. Due to a conversion of kinetic energy to internal energy upon collision, bonds break and fragments are produced. ${ }^{58}$ From the HCD cell, newly produced fragments are sent back into the C-trap for re-focusing and 
subsequently injected into the Orbitrap for the final mass analysis and production of the mass spectrum. ${ }^{58}$ The Orbitrap mass analyzer is an electrostatic device that consists of a central, spindle-shaped electrode that ions oscillate around. ${ }^{59}$ By detecting axial motion around the inner electrode the signal produced is Fourier-transformed yielding high resolution mass spectra. ${ }^{24-27}$ Operation of the Q-Exactive in full scan mode does not utilize the HCD cell. Simply switching operation modes to the all ion fragmentation (AIF) mode provides the capability for MS/MS analysis.

The outer electrode is split up symmetrically serving as a sensor surrounding the central electrode. ${ }^{56}$ Detection of the ions occurs via image current detection whereby the encapsulating outer electrodes maps the current induced by axial motion of ions around the central cylindrical electrode. ${ }^{56}$ The data collected by the sensors is converted to $\mathrm{m} / \mathrm{z}$ by Fourier transformation.

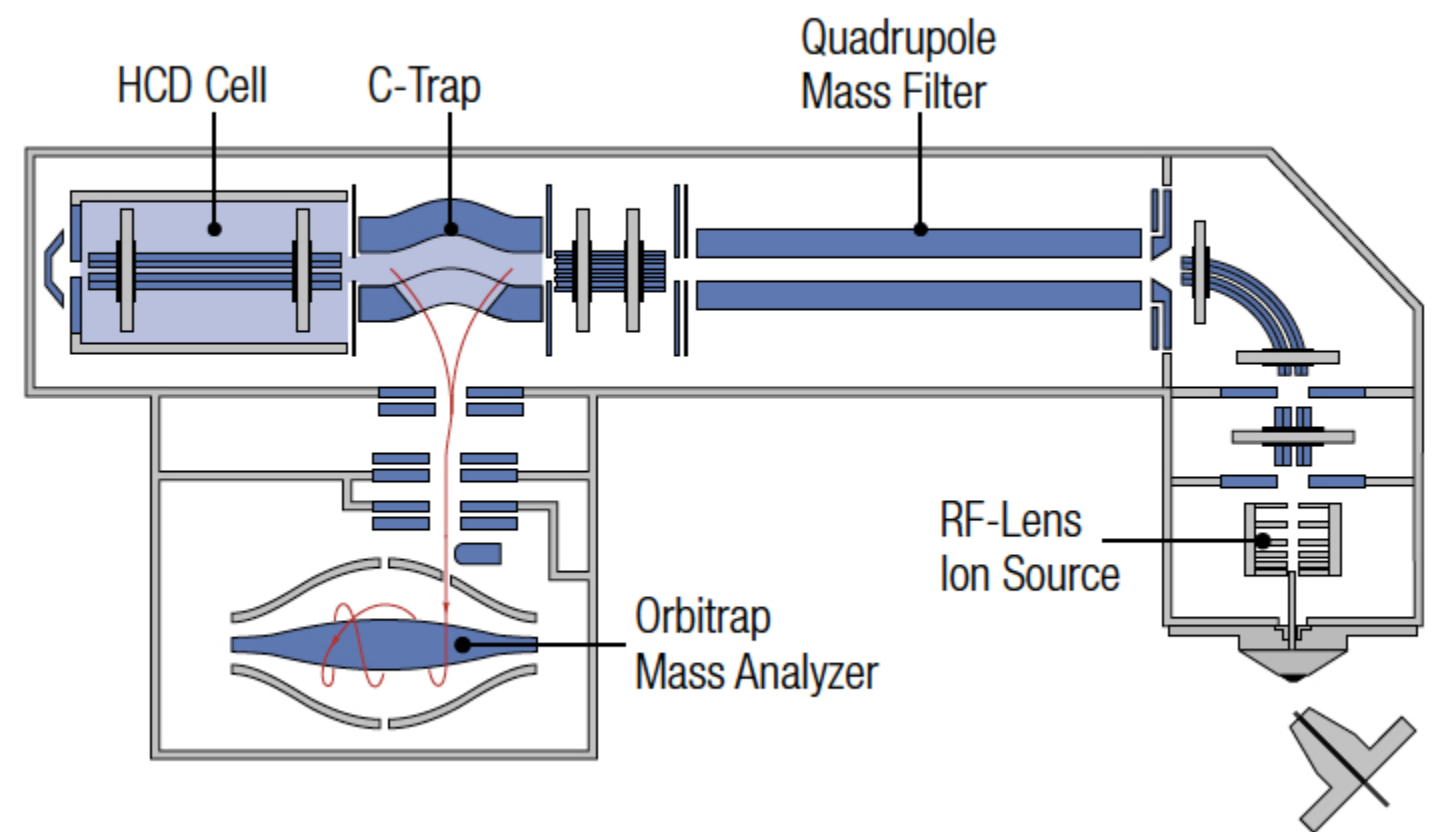

Figure 14. Schematic diagram of the design and engineering of the Q-Exactive hybrid mass spectrometer. 
Due to the complex sample matrix created when a blast occurs, it is important to demonstrate and verify the capability of ambient MS techniques to identify explosive residues from post-blast samples with appropriate sensitivity and selectivity required by courts of law. The scope of this thesis comprises an exploration into the applicability of DART-MS to characterize typical homemade explosives (HME) used in simulated improvised explosive devices (IEDs). 


\section{Materials and Methods}

\subsection{Consumables, Reagents and Standard Reference Materials}

All consumables, reagents and solvents purchased were used as received. ACS grade or better acetone (>99.7\%, Caledon Laboratories Ltd., Georgetown, ON), methanol ( $>99.8 \%$, Caledon Laboratories Ltd., Georgetown, ON) and acetonitrile ( $\geq 99.9 \%$, SigmaAldrich, Oakville, ON) were used as solvents; hydrogen peroxide (50 wt. \%, Sigma-Aldrich, Oakville, ON), sulfuric acid (95.0 -98.0\%, Caledon Laboratories Ltd, Georgetown, ON), citric acid ( $\geq 99.5 \%$, Sigma-Aldrich, Oakville, ON), nitric acid, anhydride acetic acid, D(+)cellobiose, hexamethylenetetramine ( $\geq 99.9 \%$, Sigma-Aldrich, Oakville, ON), and methyl ethyl ketone (ACP Chemicals Inc., Montreal, Quebec) were used as HME synthesis reagents; diesel, Vaseline, lamp oil, wax, grease, sugar, dextrin, and stump remover (collectively purchased locally), ammonium nitrate, potassium perchlorate, potassium chlorate were all used as either the fuel or oxidizer source for homemade binary explosives; BlueDot single base smokeless powder and GreenDot double base smokeless powder commercial explosive products were used as purchased; certified explosive standards were purchased as solutions $\left(0.1 \mathrm{mg} \cdot \mathrm{mL}^{-1}\right.$ in either methanol or acetonitrile) from Chromatographic Specialties Inc. (Brockville, ON). 


\subsection{IED Construction, Detonation and Fragment Collection}

Multiple IEDs were assembled to yield a variety of post-blast fragments characteristic of materials commonly recovered post-blast and received at our laboratory analysis, such as: cellphones, wires, batteries, nuts, bolts, metal switches, and mechanical timers (Figure 15). For safety reasons, the container used for each IED was a thin-walled aluminum can. Each IED was configured with a main charge (Table 2) and fired using a commercial electric blasting cap (approximately 1 g PETN). TATP, HMTD, MEKP and all binary explosives were prepared by a qualified chemist, in accordance with standard best practices and in compliance with Canadian Explosives Regulations (SOR/2013-211) and characterized before use to demonstrate fit for purpose. The MEKP prepared was confirmed by LCMS analysis to contain the linear dimer, trimer and tetramer as the major constituents, and the cyclic trimer as a minor constituent. ${ }^{27}$ Bluedot single-base and GreenDot double-base smokeless powder were placed in individual vials, as purchased.
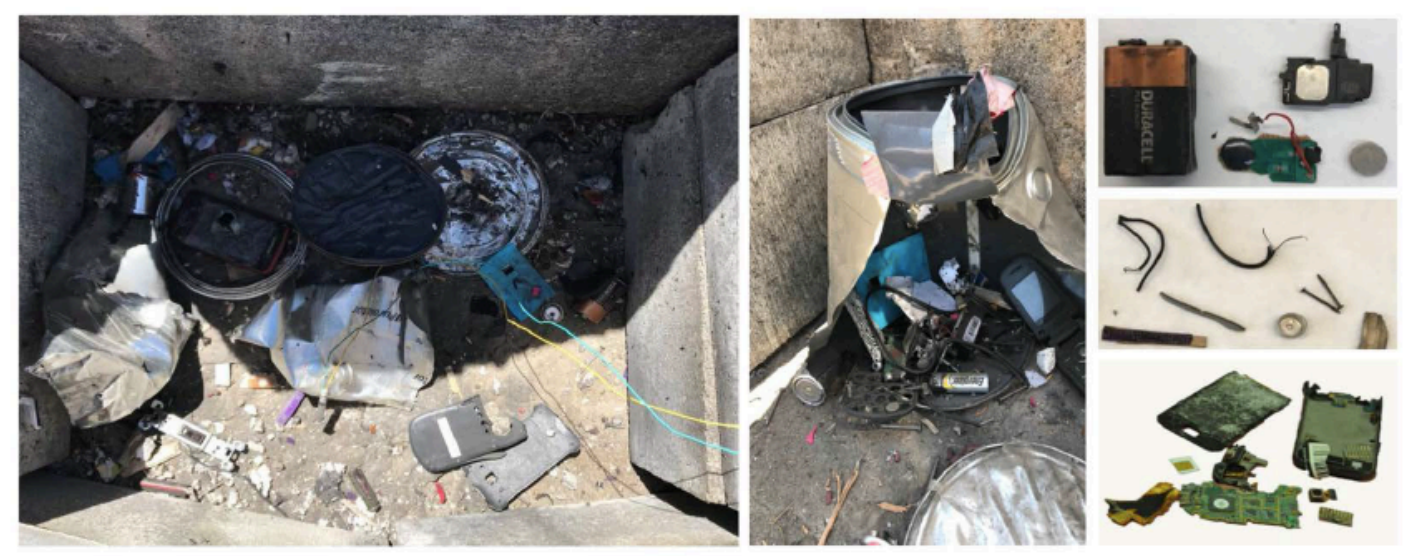

Figure 15. Fragments collected post-blast from the detonation of devices utilizing homemade explosives as the main charge (IED\# 1-14). 
To limit the spread of debris and ergonomically facilitate fragment collection, each IED was enclosed by cinder blocks before detonation. Each individual IED was remotely detonated using a command wire at Royal Canadian Mounted Police (RCMP) Technical and Protective Operations Facility (TPOF) in Ottawa, ON, by a qualified RCMP Explosive Disposal technician. Conventional contamination-prevention protocols were followed consistent-with actual crime scene practices, and all fragments collected from each IED sealed into nylon-lined evidence bags prior to transport and during storage.

Table 2. Type and amount of explosive used as main charge for each IED.

\begin{tabular}{|c|c|c|}
\hline IED \# & Main Charge & Amount (g) \\
\hline 1 & TATP & 40 \\
\hline 2 & TATP & 5 \\
\hline 3 & HMTD & 3 \\
\hline 4 & HMTD & 5 \\
\hline 5 & MEKP & 5 \\
\hline 6 & Perchlorate + Vaseline & 10 \\
\hline 7 & AN (prills) + Diesel & 10 \\
\hline 8 & AN (ground) + Wax & 10 \\
\hline 9 & Chlorate + lamp oil & 10 \\
\hline 10 & KNO $3+$ grease & 10 \\
\hline 11 & Chlorate + sugar & 10 \\
\hline 12 & KNO + dextrin & 10 \\
\hline 13 & Single base & 20 \\
\hline 14 & Double base & 20 \\
\hline
\end{tabular}




\subsection{Reference Material Sample Preparation}

\subsubsection{Fuel and Oxidizer}

The commercially available petroleum-based fuels used in the binary mixtures were obtained and analyzed unmodified. Carbohydrate-based fuel products used to prepare the binary mixtures were obtained and reference samples were prepared in deionized water. Reference samples of the oxidizers used in the devices were prepared in deionized water.

\subsubsection{Octanitrate Cellobiose Synthesis and Sample Preparation}

Octanitrate cellobiose was synthesized and used as a reference material for nitrocellulose. $\beta$-cellobiose was chosen as the starting material because it contains the same 1-4- $\beta$-linkage as observed in nitrocellulose. Nitration of $\beta$-cellobiose via reaction with anhydride acetic acid and fuming nitric acid produced the desired $\beta$-cellobiose octanitrate (ONCB). The final product was filtered, using a water aspirator, and washed multiple times with sodium bicarbonate to neutralize any residual acid. Characterization of the final product was done by FTIR and matched literature values.

Approximately $5 \mathrm{mg}$ ONCB was dissolved in $5 \mathrm{~mL}$ acetone, methanol, acetonitrile, water and dichloromethane. Additional samples of approximately the same mass of ONCB were placed in glass Petri dishes and exposed to a butane flame from a barbeque lighter. Due to the energetic properties of ONCB, the white powder-product burned to a sticky syrup like residue. The residues were subsequently collected from the Petri dishes using $5 \mathrm{~mL}$ acetone, methanol, acetonitrile, water and dichloromethane. No further purification, filtration or pre-concentration was conducted. 


\subsubsection{Smokeless Powder}

Samples of approximately $10 \mathrm{mg}$ single-base and double-base smokeless powders were prepared similarly to the preparation methods for ONCB. Samples of both smokeless powders were dissolved individually in $5 \mathrm{~mL}$ acetone, methanol, acetonitrile, water and dichloromethane. In addition, samples were ignited and the remaining residues were collected from the Petri dishes using $5 \mathrm{~mL}$ acetone, methanol, acetonitrile, water and dichloromethane. No further purification, filtration or pre-concentration was conducted.

\subsection{Post-Blast Extract Preparation}

Solvent extracts of residues from post-blast fragments is an ideal sampling method for objects too large or irregularly shaped from blast damage; which may be unsuitable to be shipped to the laboratory and/or analyzed directly in the DART sample region. Residues from the post-blast fragments for the binary explosives were collected by rinsing the fragments with hexane (petroleum based fuels) or water (sugar based fuels). Residues were collected from fragments from the smokeless powder device using acetone. Nitrogen gas was used as a mechanism to pre-concentrate the extracts when required. No pre-concentration of filtration of the extracts was included. 


\subsection{DART-MS Analysis}

Data collected in positive and negative mode was done with an lonSense ${ }^{\circledast}$ DART SVP 100 source coupled to a ThermoFisher Scientific Q Exactive ${ }^{\mathrm{TM}}$ (Orbitrap) mass spectrometer (Thermofisher Scientific, Waltham, MA). The DART source was kept at $250^{\circ}$ C and $350 \mathrm{~V}$ grid voltage. MS scan range of $\mathrm{m} / \mathrm{z} 50-700$, with a resolution of 70000 FWHM. Thermo Scientific XCalibur software was used for data collection and QualBrowser software for qualitative data interpretation. Full scan acquisition was used and no dopant was added to promote adduct formation. Samples were probed using three different DART configurations; direct analysis, analysis of swabs and extracts. Fragments were analyzed directly by placing them in the sampling region for analysis at $45^{\circ}$, whereas swabs and extracts were analyzed at $0^{\circ}$. Rectangular cotton swabs $(4 \times 4 \mathrm{~cm}$, Smiths Detection, Mississauga, ON) were used to wipe fragments, and were collected either dry or moistened with a single solvent: acetone, methanol or acetonitrile. Swabs with residue sub-sampled from fragments were then analyzed by placing them in the sampling region. Extracts were deposited onto an Ion Sense Quickstrip (consumable card with stainless steel mesh wells), placed into a card holder sitting on a mechanical rail, responsible for moving the samples to and from the sampling region. 


\section{Results and Discussion}

\subsection{DART-MS Parameter Optimization}

Prior to challenging the application of DART-MS to identify HME residues postblast, a bench level method was validated in the laboratory. Target explosives were analyzed in replicate using a QuickStrip card while systematically varying DART and MS settings to ensure reproducibility and robustness of detection. Parameters optimized included temperature of the DART probe, distance between the DART and MS and resolution of the Orbitrap. Limit of detection (LOD) was evaluated to determine the lowest quantity of explosives resulting in observation of three or more characteristic ions

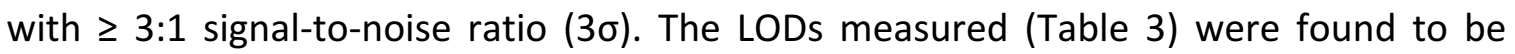
comparable to reported values. ${ }^{44}$ Once satisfied with the DART-MS parameters and the method design, the applicability to detect explosive residues from the post-blast bomb fragments and identify the variety HME used in the devices was investigated.

Table 3. LODs observed for explosives of interest measured using in-house method and QuickStrip compared to literature values.

\begin{tabular}{|c|c|c|}
\hline Explosives & Measured LOD (ng) & Reported $^{45}$ LOD (ng) \\
\hline TNT & 0.01 & 0.25 \\
\hline HMX & 0.10 & 10 \\
\hline RDX & 0.01 & 0.50 \\
\hline PETN & 0.10 & 5 \\
\hline Tetryl & 0.10 & 1 \\
\hline NG & 1 & 5 \\
\hline $2,4-D N T$ & 10 & 0.50 \\
\hline $2,6-D N T$ & 10 & 0.50 \\
\hline EGDN & 100 & 100 \\
\hline HMTD & 1 & - \\
\hline TATP & 100 & - \\
\hline MEKP & 100 & - \\
\hline
\end{tabular}




\subsection{Analysis of Fragments}

Due to the design and capability of the DART-MS method, multiple sampling regimes (e.g. direct, in-direct and extracts) were evaluated to determine efficacy of residue recovery. Each sampling method revealed the HME used, however different recovery efficiencies were exposed. Aside from minor differences in relative abundance of ions, sample-to-sample, the mass spectra and corresponding fragmentation patterns for all explosives were in high agreement with the reference materials.

As expected without pre-concentration or sample clean-up prior to analysis, background ions were commonly observed in the DART-MS spectra. Spectra were collected prior, during and after each sample analysis to monitor cleanliness of the instrument and to identify background ions endogenous to the DART or the sampling technique (i.e. swabbing or use of Quickstrip) (Figure 16 - 19). Analysis of both polar and organic solvents deposited on the QuickStrip produced spectra with total ions counts ranging from $10^{4}-10^{6}$ (Figure 20 and 25). To facilitate elimination of the solvent contribution, solvent blanks were included in the analysis along with extracted samples. 


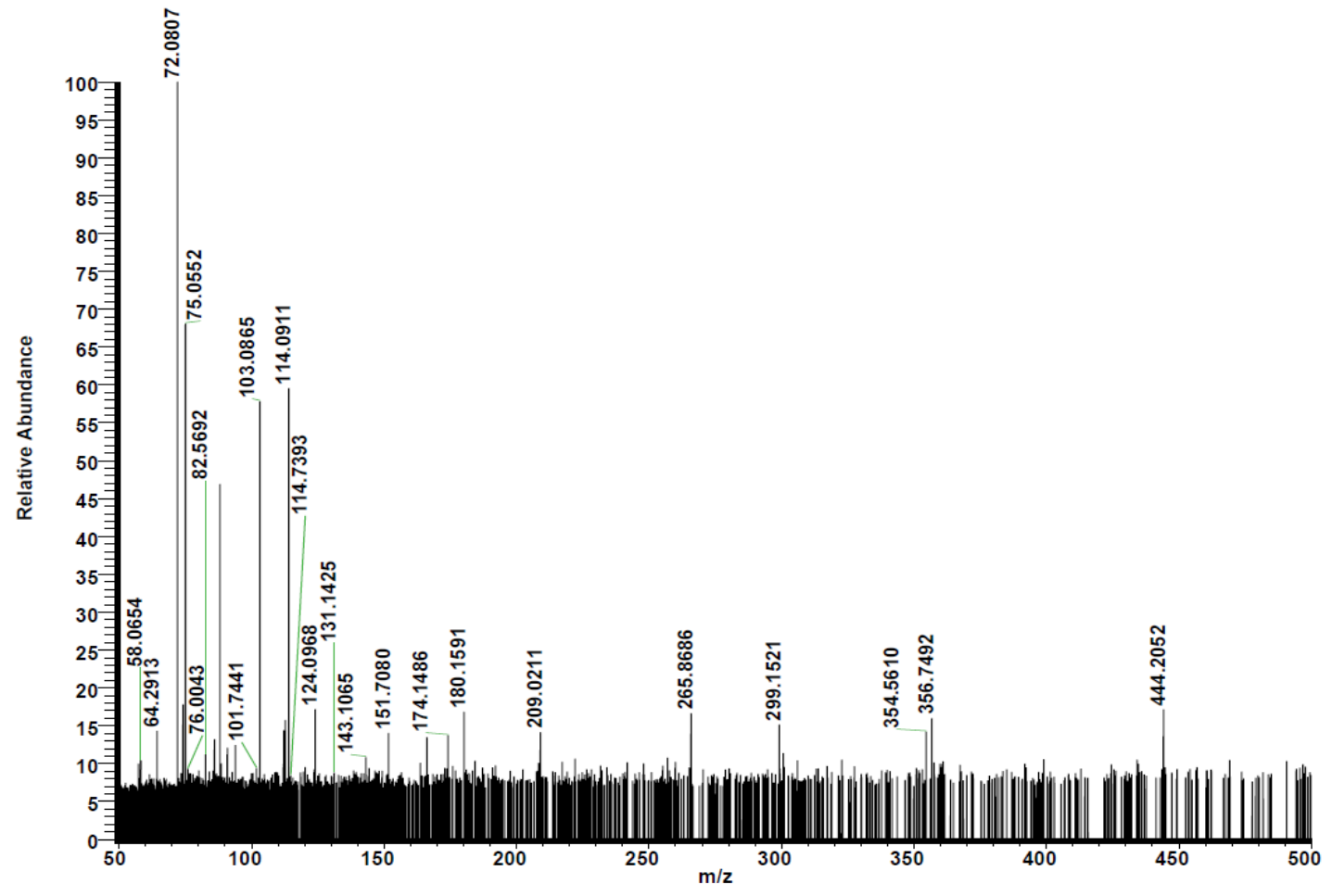

Figure 16. Ions observed via operation of the QExactive in positive full scan mode, without the DART source turned on. Total ion count $10^{3}$.

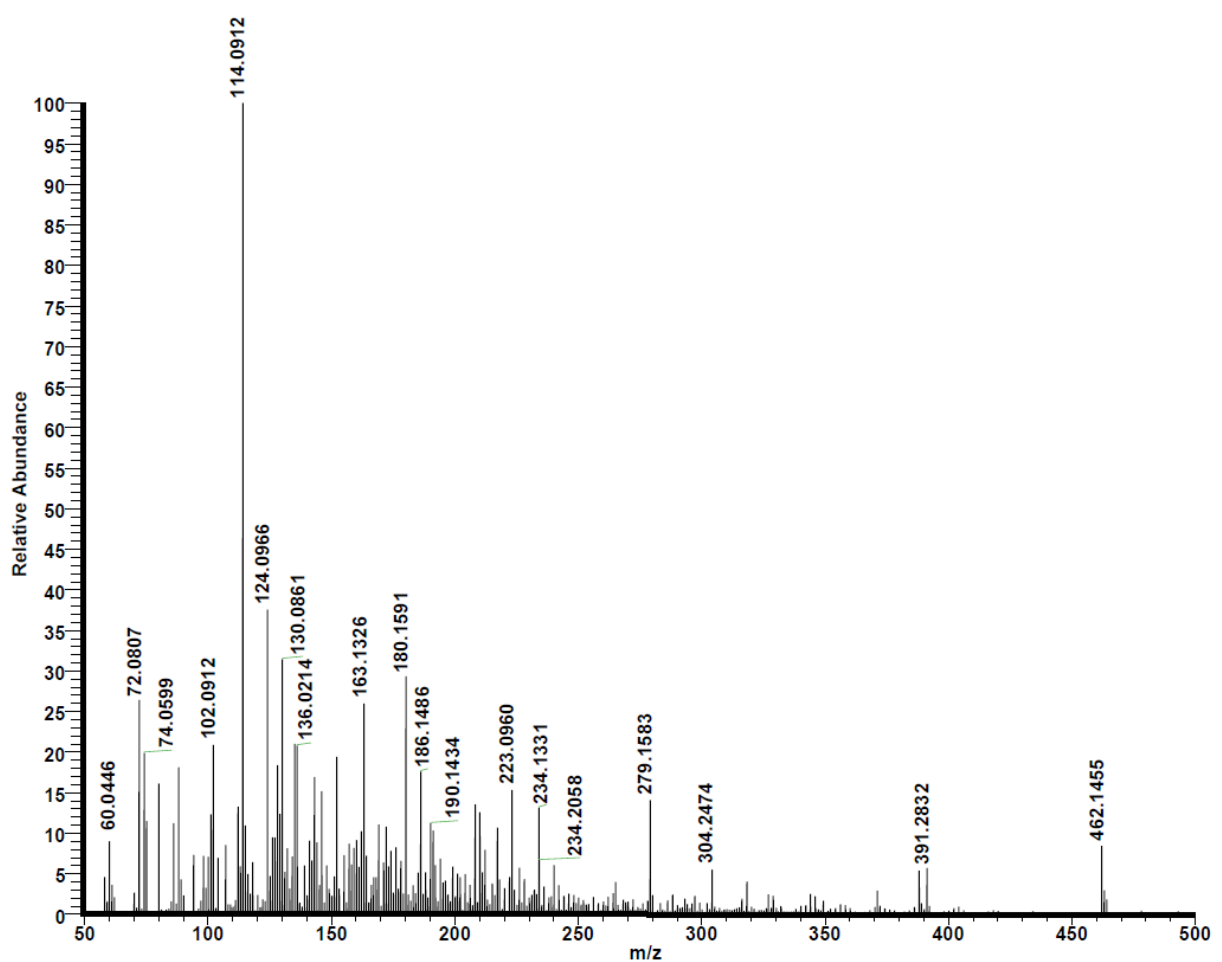

Figure 17. Endogenous DART-MS ions. Total ion count $10^{4}-10^{5}$. 


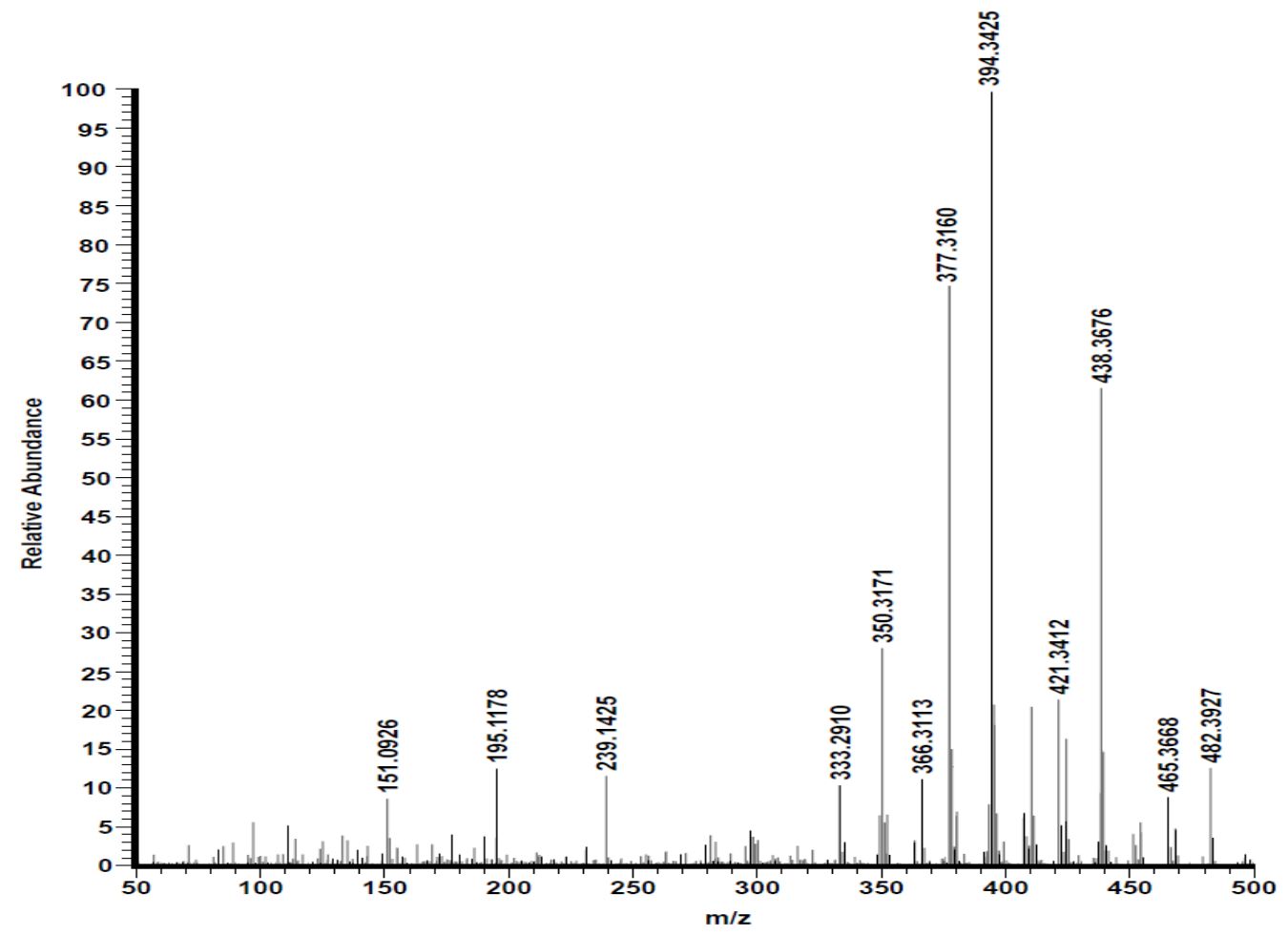

Figure 18. Analysis of an unused cotton swab in positive mode using full scan. Total ion count $10^{4}$

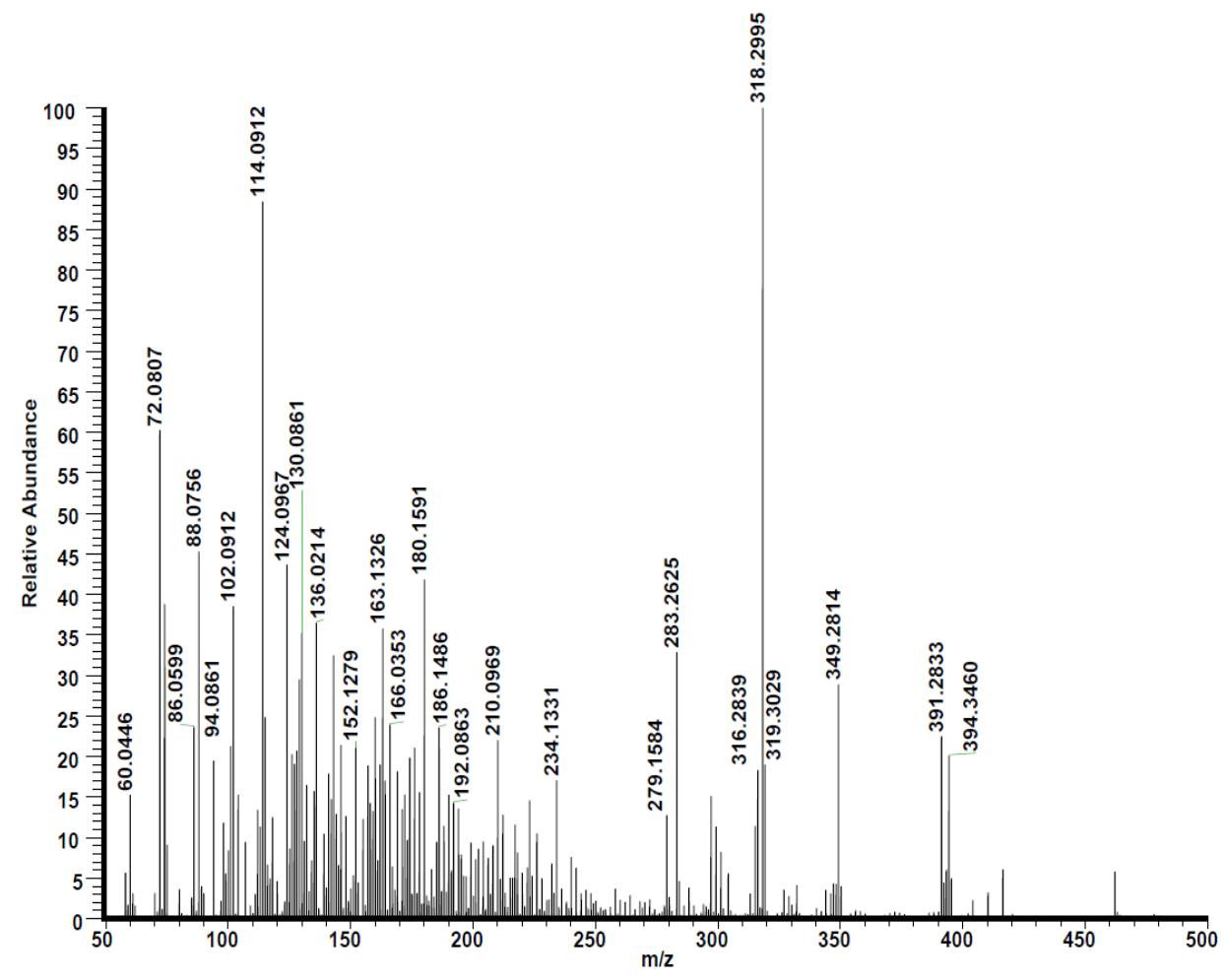

Figure 19. An unused QuickStrip analyzed in positive mode using full scan. Total ion count $10^{3}-10^{5}$. 


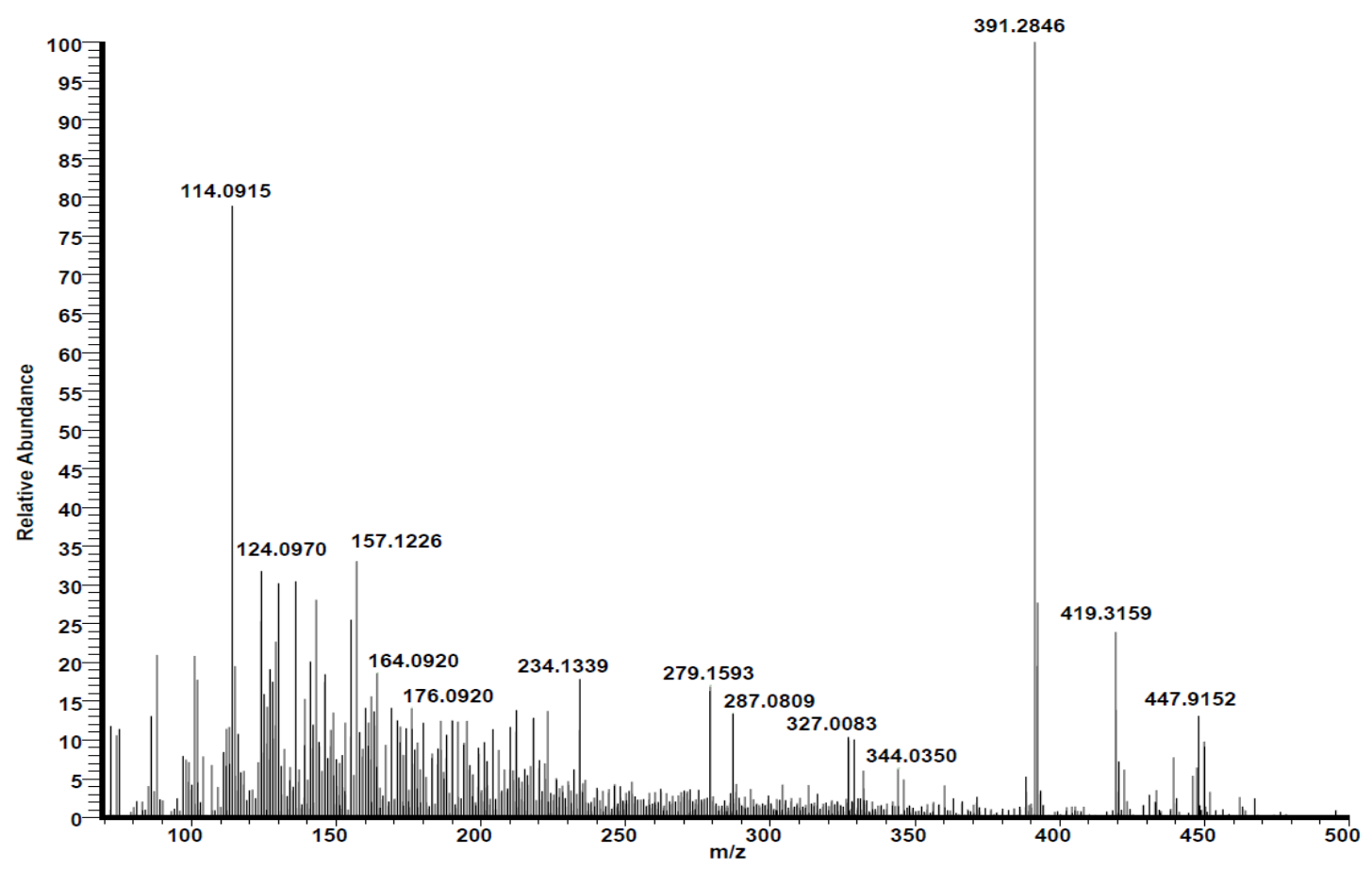

Figure 20. Water deposited onto a QuickStrip, analyzed in positive mode using full scan. Total ion count $10^{4}-10^{5}$.

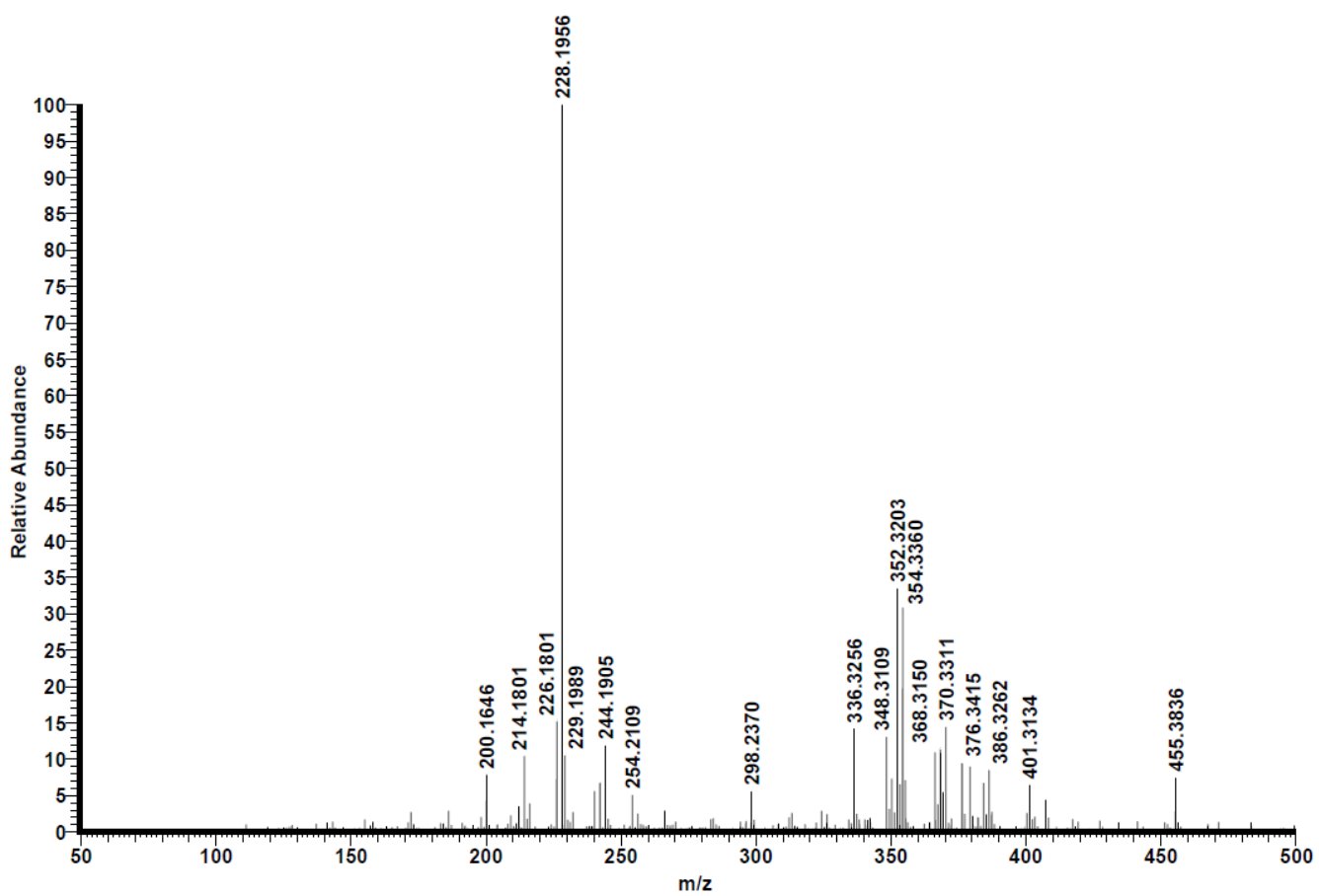

Figure 21. Methanol deposited onto a QuickStrip, analyzed in positive mode using full scan. Total ion count $10^{4}-10^{6}$. 


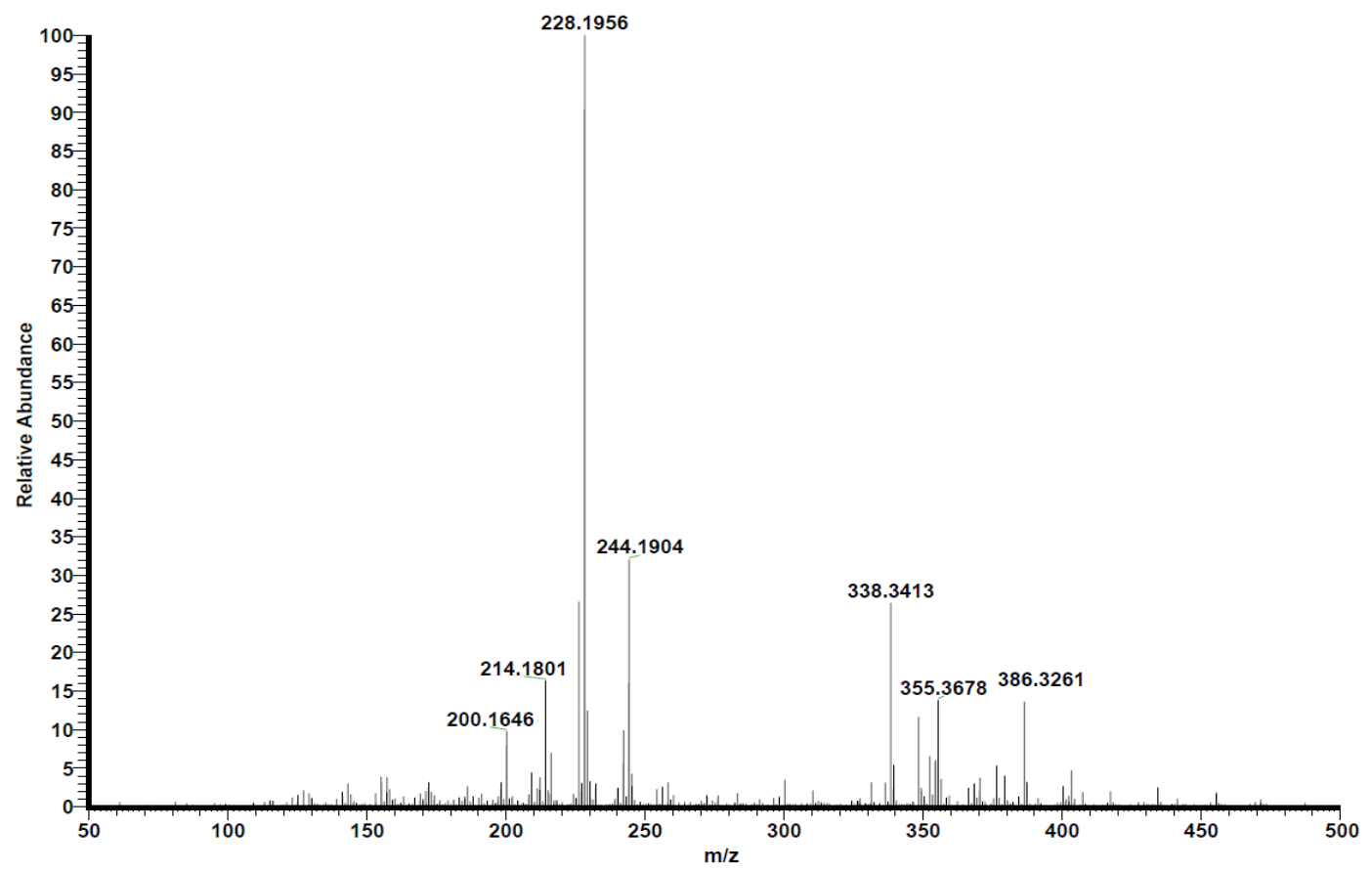

Figure 22. Acetone deposited onto a QuickStrip, analyzed in positive mode using full scan. Total ion count $10^{4}-10^{6}$.

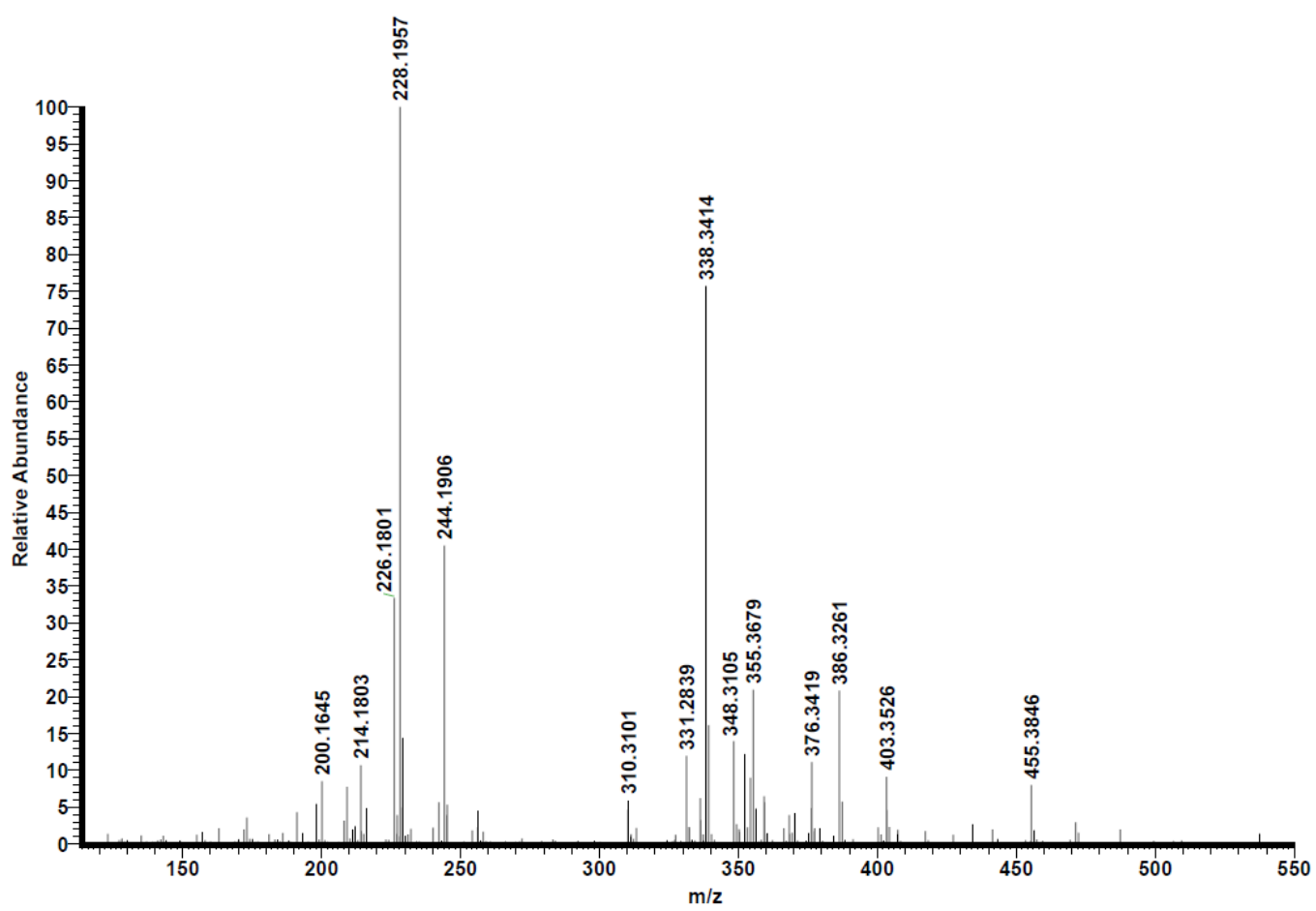

Figure 23. Acetonitrile deposited onto a QuickStrip, analzyed in positive mode using full scan. Total ion count $10^{4}-10^{6}$. 


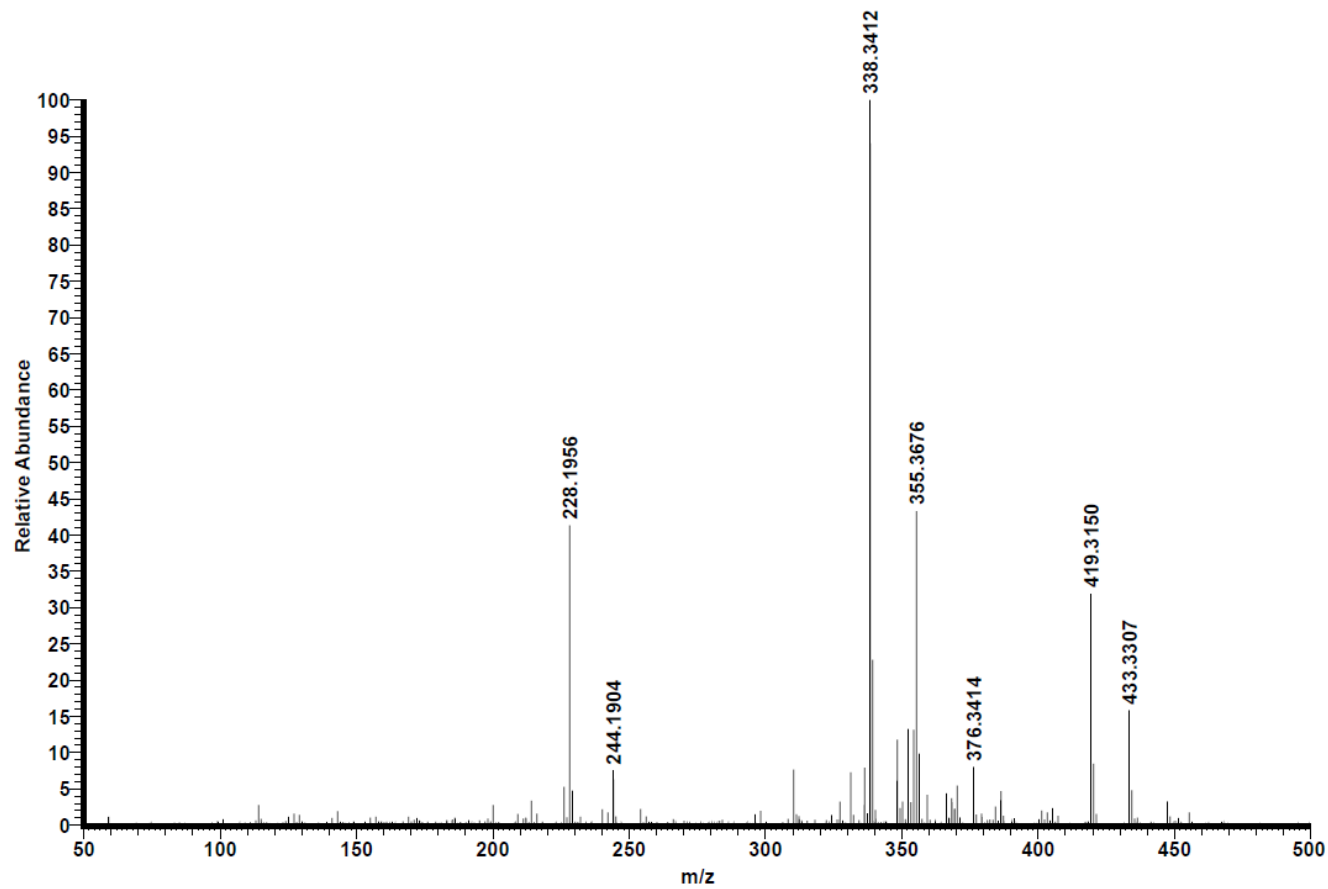

Figure 24. Analysis of dichloromethane deposited onto a QuickStrip, in positive mode using full scan, to be used for solvent associated ion subtraction. Total ion count $10^{4}-$ $10^{6}$.

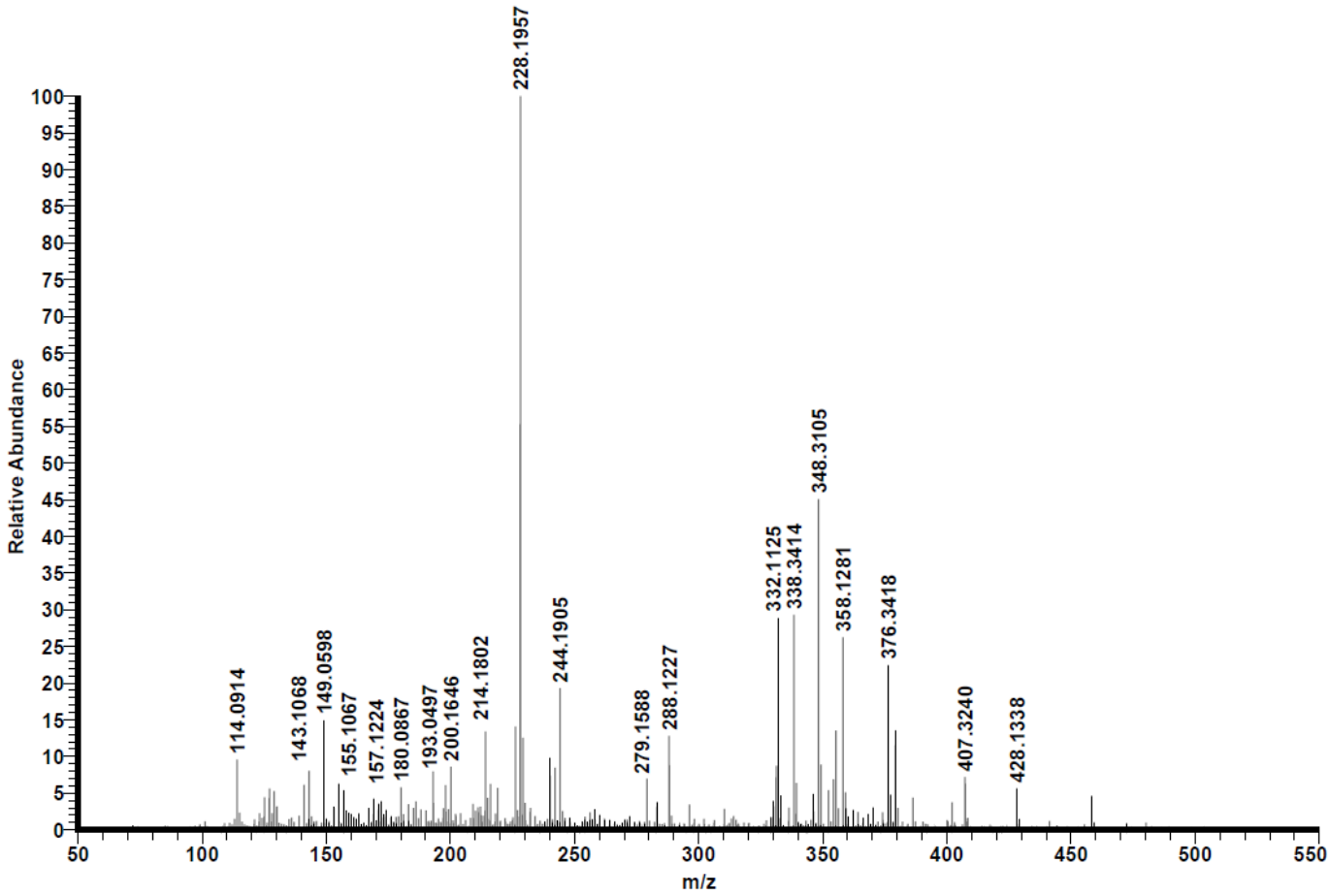

Figure 25. Analysis of hexane deposited onto a QuickStrip, in positive mode using full scan. Total ion count $10^{4}-10^{5}$. 
Detection and identification of the HME used for each IED was obtained by direct analysis of at least one fragment recovered from each device. In real case work it is common to not detect explosive residue from every bomb fragment due to the unequal geospatial distribution of residues which is a direct result of the unpredictable nature of an explosive blast. ${ }^{62}$ The physical size of the fragments found varied from small (e.g. SIM card $\sim 0.25 \mathrm{~cm}^{2}$ ) to large (e.g. D-cell battery $\sim 17.25 \mathrm{~cm}^{2}$ ), which means fragments can retain different quantities of residue. The interaction area of the DART probe will not instantly desorb all residue from surfaces, which will limit the amount of material transmitted to the MS inlet per scan. Moreover, many of the fragments from each device were in storage for at least four $(4+)$ months prior to analysis, which is not an uncommon time-frame with actual investigations. Thus, some extent of residue loss from the fragment surface(s) via degradation can be expected. ${ }^{59-61}$ Recovery efficiencies with longer exposure times of post-blast IED fragments to air or weather was not quantified, but will be given further consideration in future work.

In-direct analysis was done to evaluate compatibility of the DART-MS method with using swabs. Swabs are ideal to sub-sample objects too large to be shipped to the laboratory or irregularly shaped fragments unsuitable for direct analysis. Analyzing a fragment directly may also contribute to higher background in the MS if the substrate of the fragment itself can be desorbed and ionized by the DART, making swabs an attractive alternative. In addition, swabbing is suited to accumulate more residue for desorption by the DART probe and transmission to the MS inlet per scan, which will enhance recovery and detection. Efforts to maximize residue collection using swabs included evaluating 
commercially available swabs manufactured with different substrates as well as comparing collection efficiency and effectiveness when using dry or solvent-dampened swabs.

Solvent extraction was convenient to recover residue from a fragment when direct analysis was not suitable. Liquid extracts can be filtered and/or pre-concentration as a mechanism to improve detection. Common best practices for forensic sampling of explosives residues recommend either acetone or methanol be used as the solvent. Acetone, methanol $(\mathrm{MeOH})$, water, acetonitrile (ACN), dichloromethane (DCM), and hexane were evaluated as solvents to extract post-blast residues while minimally coextracting environmental congeners. One drawback of using polar organic solvents is the potential for co-extraction of other substances and contaminants from the substrate/bomb fragment. In general, organic solvents unselectively extract any organic substances present from a specimen.

Substrate ionization can occur with plastics ${ }^{64}$ and textiles ${ }^{65,66}$ which will also contribute unwanted spectral background. Fragments representing different materials recovered post-blast (e.g. plastic, metal, rubber, etc.) were thus surveyed to determine possible spectral contribution from the different substrates themselves. No explicit variation was observed between different fragments to indicate any one substrate was preferentially ionized over another, indicating the background ions observed could not be distinguished from substrate or environmental contamination when recovered from the ground. Fortunately, this is probative for our verification purposes, because real specimens from post-blast debris are expected to be dirty from environmental 
contamination and unwanted substances are often observed in the matrix of forensic samples. To visually enhance where the target HMEs were detected in the total DART-MS desorption profile observed for each fragment, extracted ion chromatograms (EIC) were found particularly useful to visually isolate regions of interest. High mass accuracy permitted assured reliable identification of all HME.

\subsubsection{Organic Peroxide Explosives}

TATP and HMTD are the most common OPBE encountered in case work at Canadian forensic laboratories. MEKP is less common, but is easily prepared analogous to TATP or HMTD and it remains a priority to ensure standard methods can detect MEKP post-blast as well. Via both direct and in-direct sampling methods, DART-MS analysis revealed the OPBE used from at least one fragment recovered from each device (Table 4). Analysis of swabs was generally found to be more effective than by direct analysis of fragments, except for MEKP, which proved the most challenging peroxide HME to sufficiently detect post-blast (Table 4). 
Table 4. Depiction of the OPBE identified via DART-MS direct analysis of a multitude of different post-blast fragments compared to in-direct.
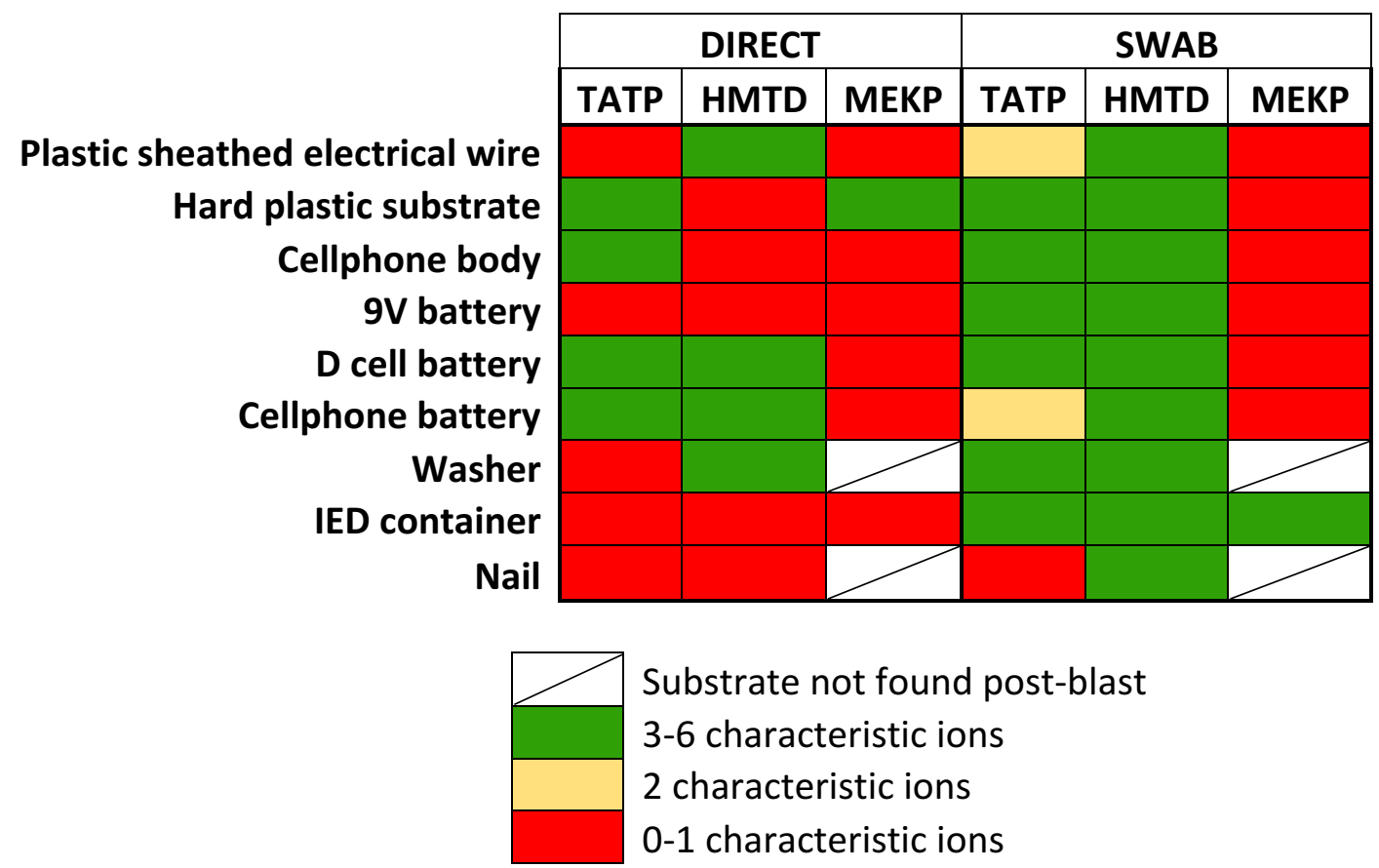

TATP was positively identified as the explosive used in IED \#1 and 2 via direct and in-direct analysis of the post-blast fragments (Figure 27 and 28). Identification was offered by observing the following ions identified as characteristic via analysis of TATP certified reference material (Figure 26); ammoniated TATP molecular ion (m/z 240.1436 $\left.\left[\mathrm{M}+\mathrm{NH}_{4}\right]^{+}\right)$, mono-acetone peroxide $\left(\mathrm{m} / \mathrm{z} 74.0364[\mathrm{M} / 3]^{+}\right)$and protonated fragments from the parent trimer $\mathrm{m} / \mathrm{z} 75.4406\left[\mathrm{C}_{3} \mathrm{H}_{6} \mathrm{O}_{2}+\mathrm{H}\right]^{+}, 89.0597\left[\mathrm{C}_{4} \mathrm{H}_{9} \mathrm{O}_{2}\right]^{+}$and $91.0390\left[\mathrm{C}_{3} \mathrm{H}_{6} \mathrm{O}_{3}+\right.$ $\mathrm{H}]^{+}$. The base peak observed for TATP from analysis-to-analysis was either the $\mathrm{m} / \mathrm{z}$ 91.0390 fragment or $\mathrm{m} / \mathrm{z} 240.1436\left[\mathrm{M}+\mathrm{NH}_{4}\right]^{+}$. Characteristic ions for TATP were consistently detected with an abundance of $10^{5}-10^{7}$ counts and with spectral background ions typically between $10^{3}-10^{4}$ counts. The amount of TATP used in IED \#1 versus \#2 was deliberately chosen as it replicated past case work scenarios examined. 
Quantities of TATP ranging from $10^{1}-10^{3}$ gram scale (i.e. small and large scale) have been encountered in criminal investigations, where larger amounts were manufactured to be used as demolition charge(s) ${ }^{21}$ and smaller amounts for concealment in victim-operated IEDs. ${ }^{22}$ The similar abundance of ions observed for TATP detected from both IED \#1 and \#2 is indicative that sufficient residue was retained on certain fragments from IED \#2 to be detected in similar quantity to that recovered from IED \#1. The maximum signal response for the MS is $10^{8}$ counts, which indicates the ions detected for TATP from either device did not saturate the detector. Retaining sufficient TATP for detection was not unexpected because all fragments were collected soon after detonation and wellpreserved in air-tight evidence collection bags to optimize the opportunity for $\mathrm{HME}$ residue recovery.

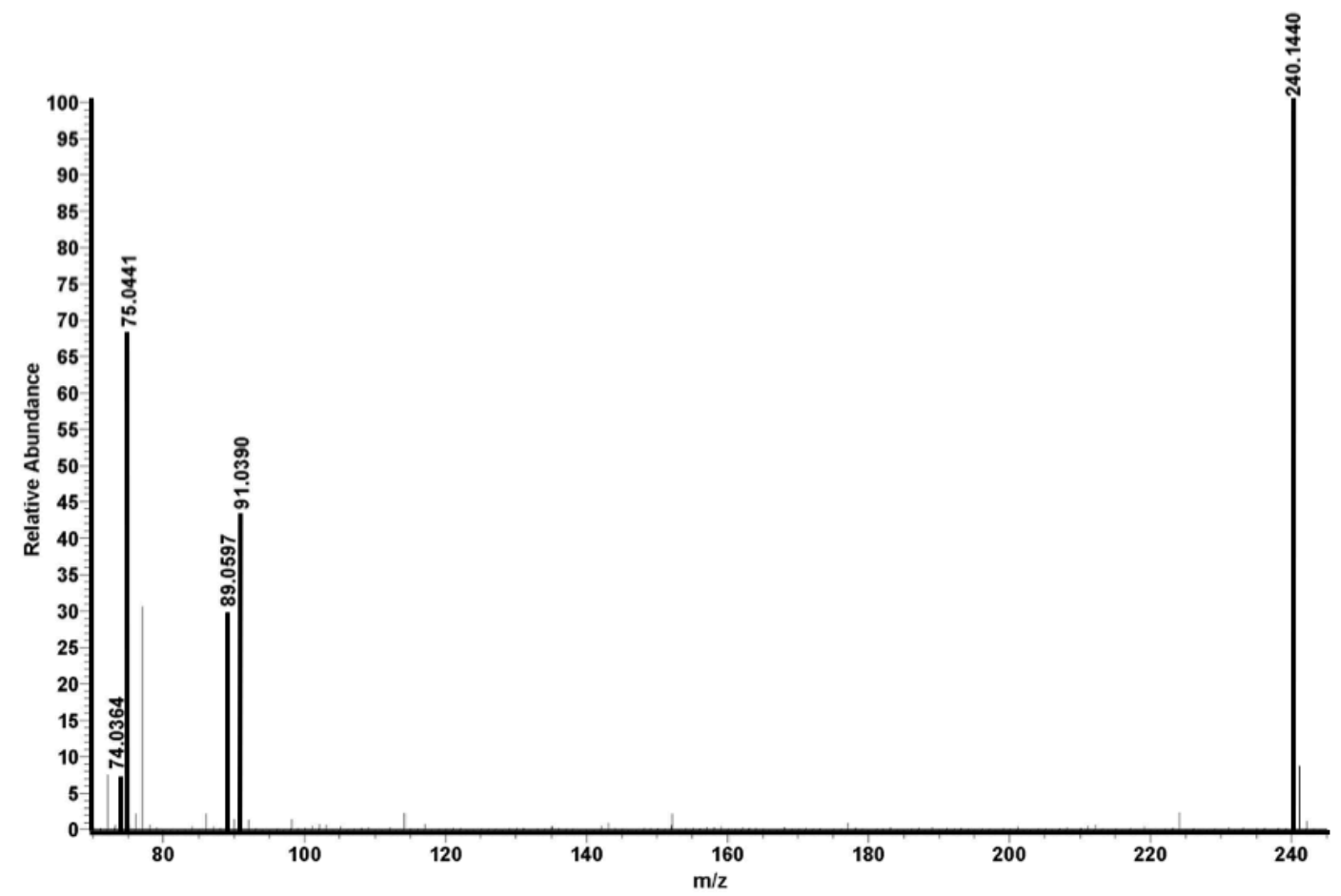

Figure 26. Positive mode, full scan high-resolution mass spectrum for TATP analyzed from certified reference standard. Ions characteristic of TATP have been bolded and labelled. 


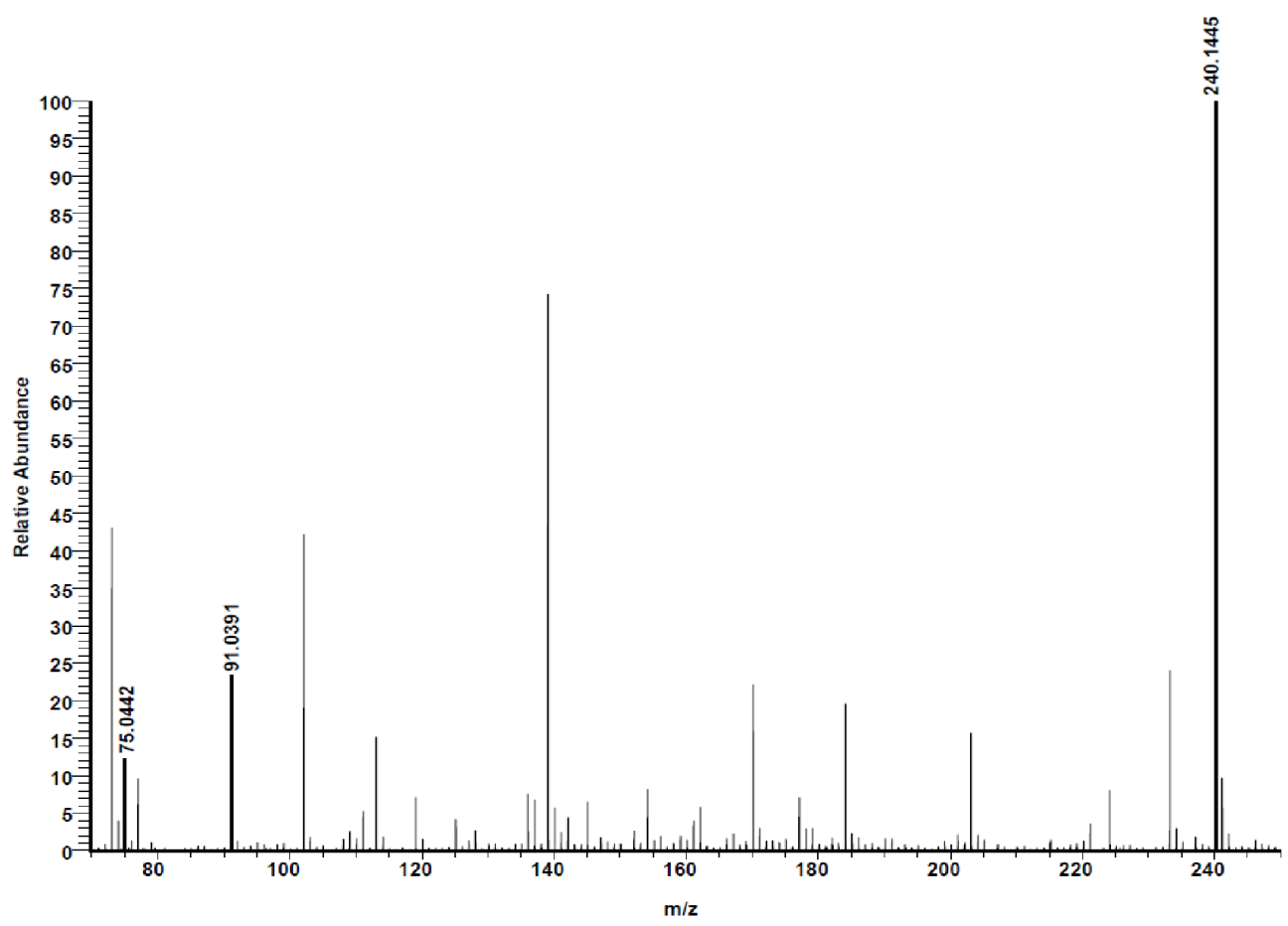

Figure 27. Positive mode, full scan high-resolution mass spectrum for TATP upon direct analysis of fragment from IED \#2. Ions characteristic of TATP have been bolded and labelled.

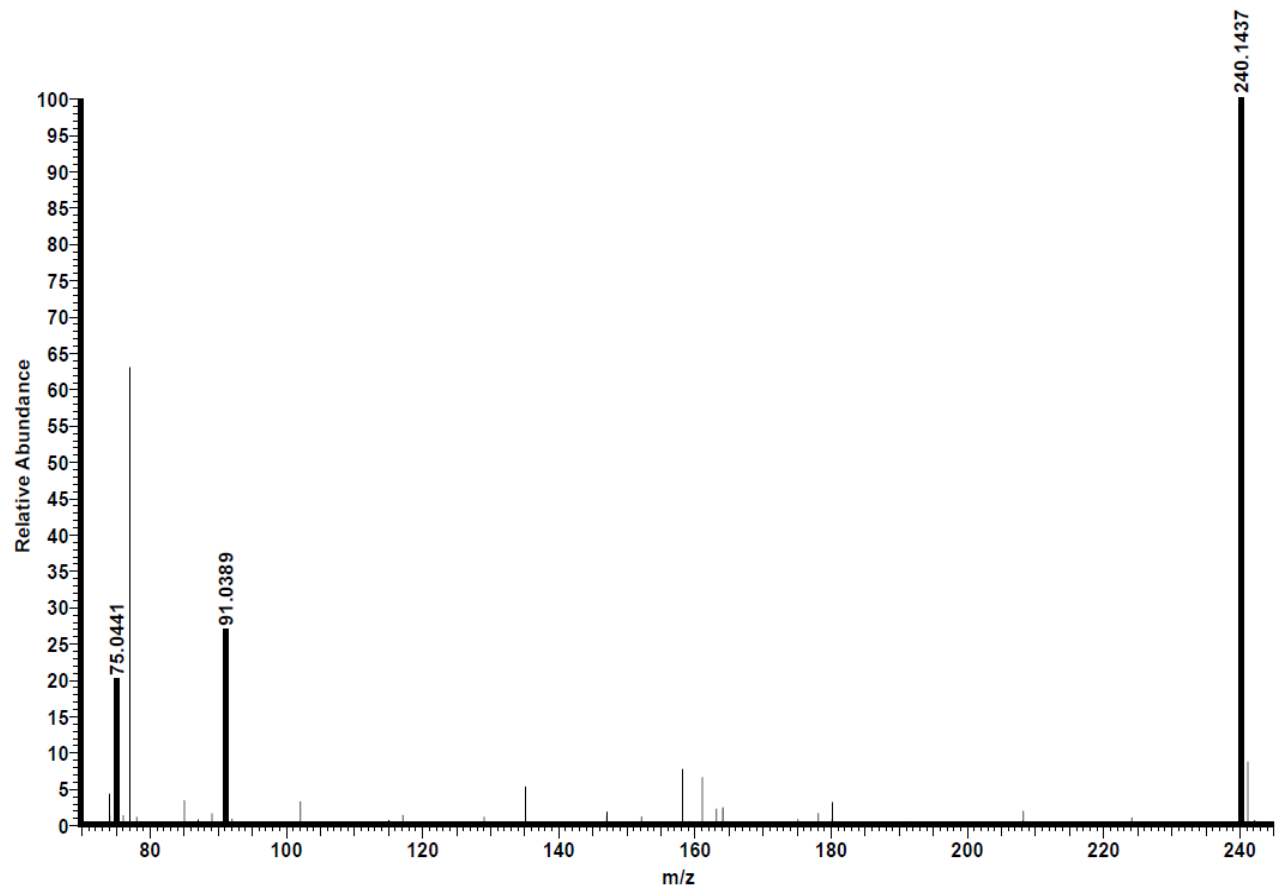

Figure 28. Positive mode, full scan high-resolution mass spectrum for TATP analyzed from a swab used to collect post-blast residues from IED \#2 fragments. Ions characteristic of TATP have been bolded and labelled. 
HMTD was identified as the main charge for IED \#3 via observation of characteristic ions identified in analysis of HMTD certified reference material (Figures 2931); protonated molecular ion $\left(\mathrm{m} / \mathrm{z} 209.0768[\mathrm{M}+\mathrm{H}]^{+}\right)$and commonly observed fragments 9, $42 \mathrm{~m} / \mathrm{z} 88.0394\left[\mathrm{M}-\mathrm{C}_{3} \mathrm{H}_{6} \mathrm{NO}_{4}\right]^{+}, 145.06081\left[\mathrm{M}-\mathrm{CH}_{2} \mathrm{O}_{3}+\mathrm{H}\right]^{+}, 179.0663\left[\mathrm{M}-\mathrm{CH}_{2} \mathrm{O}+\mathrm{H}\right]^{+}$, 191.0664 $[\mathrm{M}-\mathrm{H}-\mathrm{O}]^{+}$and $224.0878\left[\mathrm{M}-\mathrm{H}_{2}+\mathrm{NH}_{4}\right]^{+}$. The major ion observed for HMTD analysis-to-analysis was either the $\mathrm{m} / \mathrm{z} 145.06077$ or 224.0878 fragments. Characteristic ions for HMTD were consistently detected with an absolute abundance ranging from $10^{5}$ $-10^{6}$ and with background ions remaining between $10^{3}-10^{4}$ counts. The quantity of HMTD selected for IED \#3 agrees with past case work examples, where HMTD has been encountered as filler used for homemade detonators. An ion with m/z 207.0979 was not reproducibly observed, consistent with previous reports. ${ }^{9,63}$

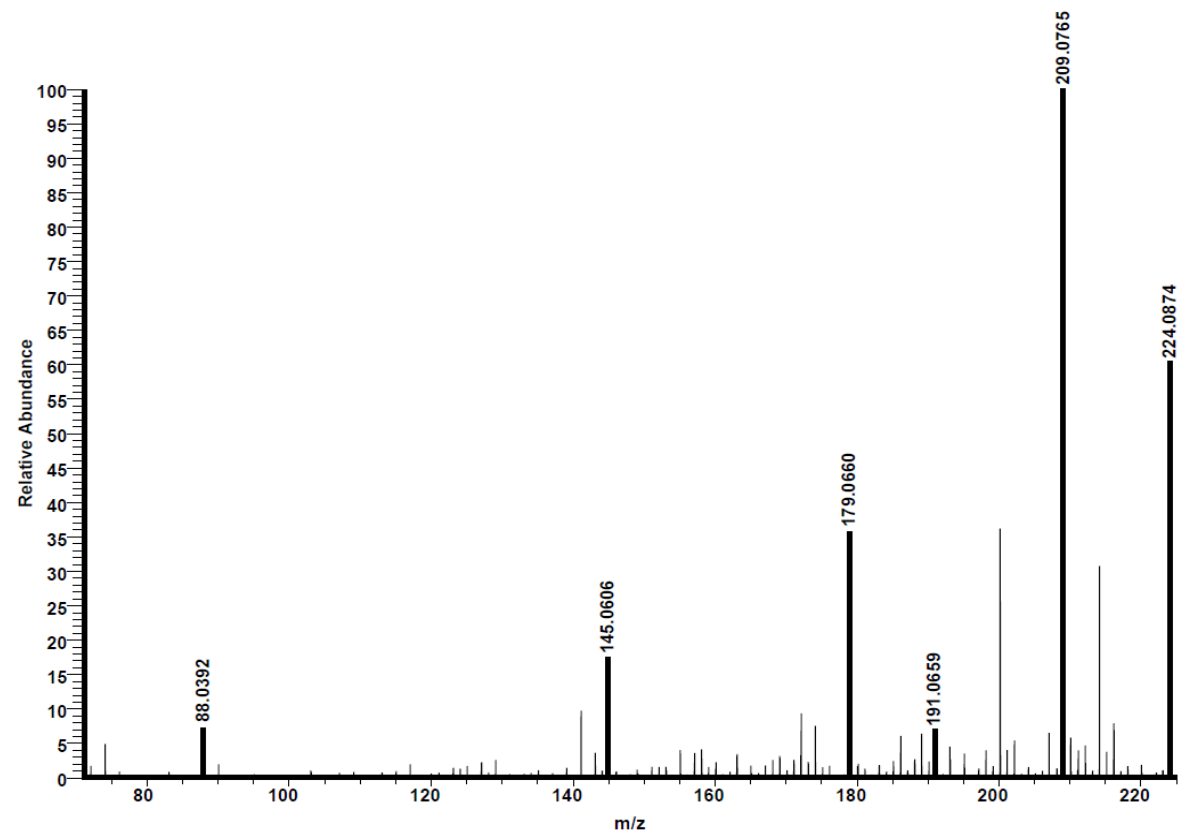

Figure 29. Full scan high-resolution mass spectrum for HMTD analyzed from certified reference. lons characteristic of HMTD have been bolded and labelled. 


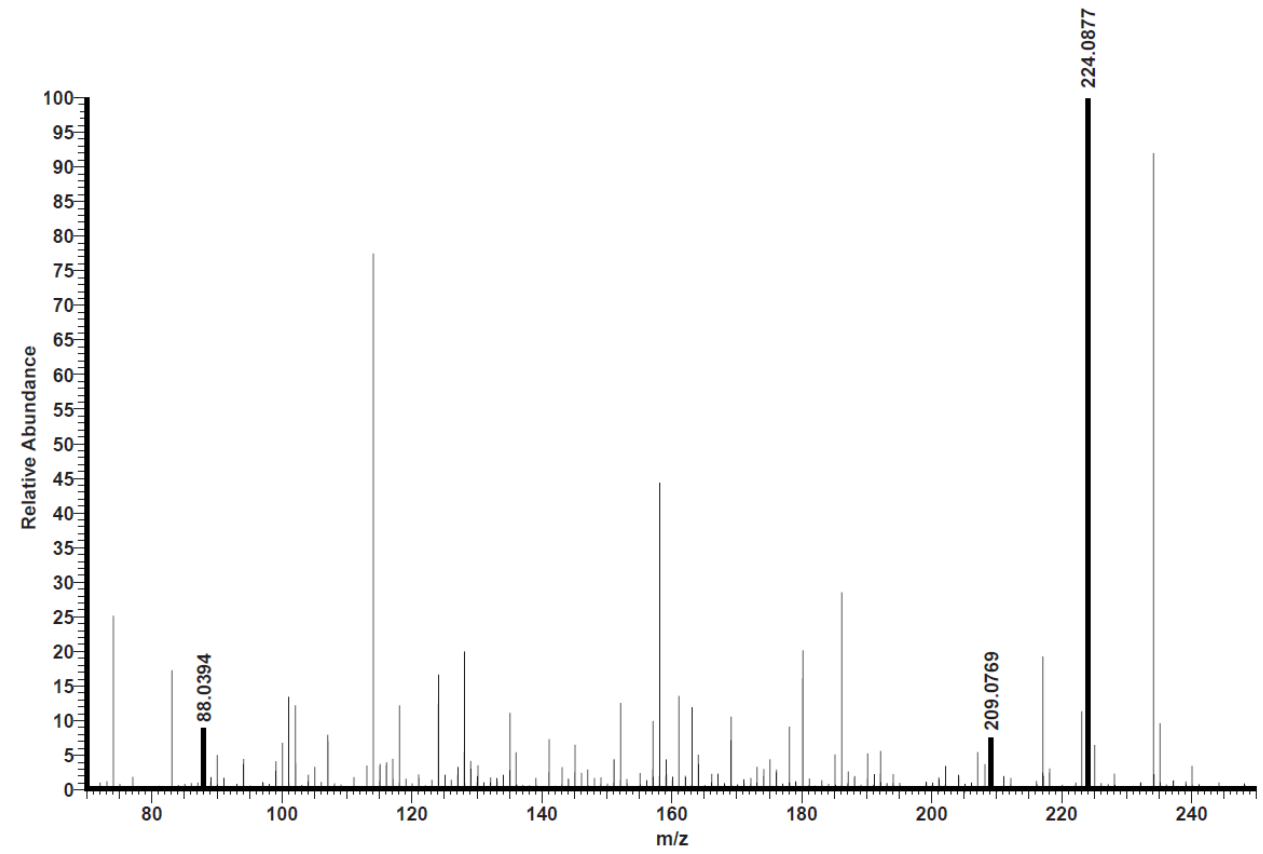

Figure 30. Full scan high-resolution mass spectrum depicting identification of HMTD from direct analysis of a fragment collected post-blast from IED \#3. Ions characteristic of HMTD have been bolded and labelled.

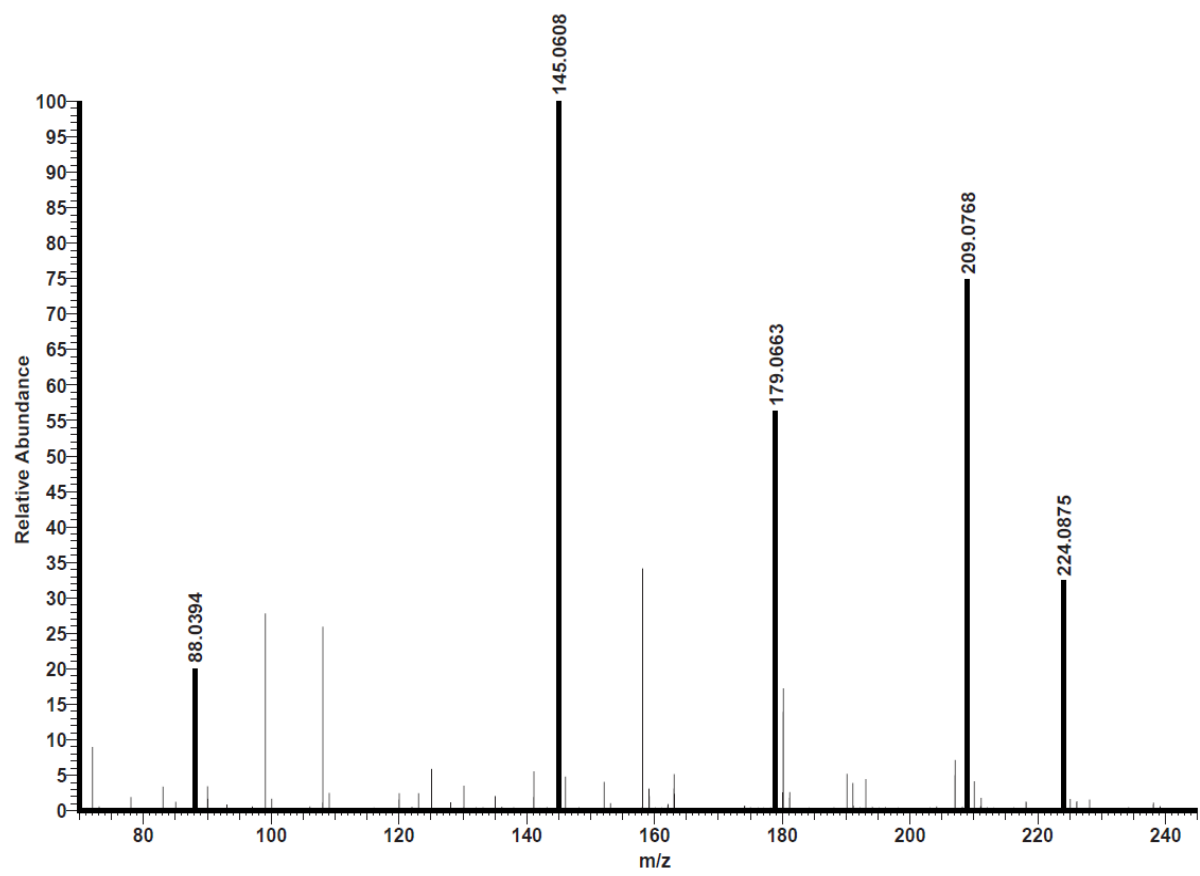

Figure 31. Full scan high-resolution mass spectrum depicting identification of HMTD upon analysis of a swab used to collect post-blast residues from IED \#3 fragments. Ions characteristic of HMTD have been bolded and labelled. 
When synthesized and left unseparated by chromatography, MEKP is a mixture of monomeric, dimeric, trimeric (linear and cyclic) and higher oligomeric species. ${ }^{39}$ For realism, we prepared MEKP and only isolated from unreacted reagents, but did not purify further before use. DART-MS analysis of our MEKP reference material yielded four characteristic ions (Figure 32); ammoniated linear trimer $\left(\mathrm{m} / \mathrm{z} 316.1965\left[\mathrm{C}_{12} \mathrm{H}_{26} \mathrm{O}_{8}+\right.\right.$ $\left.\left.\mathrm{NH}_{4}\right]^{+}\right)$, ammoniated linear tetramer $\left(\mathrm{m} / \mathrm{z} 404.2488\left[\mathrm{C}_{16} \mathrm{H}_{34} \mathrm{O}_{10}+\mathrm{NH}_{4}\right]^{+}\right)$, and monomeric fragments $\mathrm{m} / \mathrm{z} 77.0233\left[\mathrm{C}_{2} \mathrm{H}_{5} \mathrm{O}_{3}\right]^{+}$and $89.0597\left[\mathrm{C}_{4} \mathrm{H}_{9} \mathrm{O}_{2}\right]^{+}$. Characteristic ions were detected with an absolute abundance of $10^{5}-10^{6}$ and spectral background ions remained between $10^{3}-10^{4}$ counts. The ion observed at $\mathrm{m} / \mathrm{z} 316.1965$ is best calculated as corresponding to a linear trimer adduct with ammonium $\left[\mathrm{C}_{12} \mathrm{H}_{26} \mathrm{O}_{8}+\mathrm{NH}_{4}\right]^{+}$and not a monomer adduct as previously reported. ${ }^{43}$ Parent ions attributable to either the monomer or dimer species were not observed. The desorption profile observed for MEKP by DART-MS was broad and the relative ratios of ions observed changed from beginning to end. Initially, the lower mass fragments $\mathrm{m} / \mathrm{z} 77.0233$ and 89.0597 were dominantly favoured at the beginning of the scan, but transitioned to predominantly the higher mass ions 316.1965 and $\mathrm{m} / \mathrm{z} 404.2488$ towards the end. The fragment ions $\mathrm{m} / \mathrm{z} 77.0233$ and 89.0597 are common to both the monomer and dimer, suggesting a pseudo-distillation profile is occurring, where the lighter MEKP monomer and dimer species are desorbed and ionized before the heavier oligomers, but the parent ions were simply not stable enough to be observed. When the average MS is plotted over all scans in the broad desorption profile, the major parent ions observed from analysis-to-analysis were the ammonium adduct of either the trimer ( $\mathrm{m} / \mathrm{z} 316.1965)$ or tetramer ( $\mathrm{m} / \mathrm{z} 404.2488)$. We 
did not elucidate further if the monomer or dimer parent ions may be present, because detection of the four ions observed is sufficient for identification of MEKP in a post-blast residue. MEKP was identified as the main charge for IED \#5 as the same characteristic ions identified in analysis of the crude MEKP product were observed in the spectra obtained from analysis of direct and in-direct sampling techniques (Figure 33 and 34). MEKP was the most challenging peroxide HME to detect post-blast (Table 3).

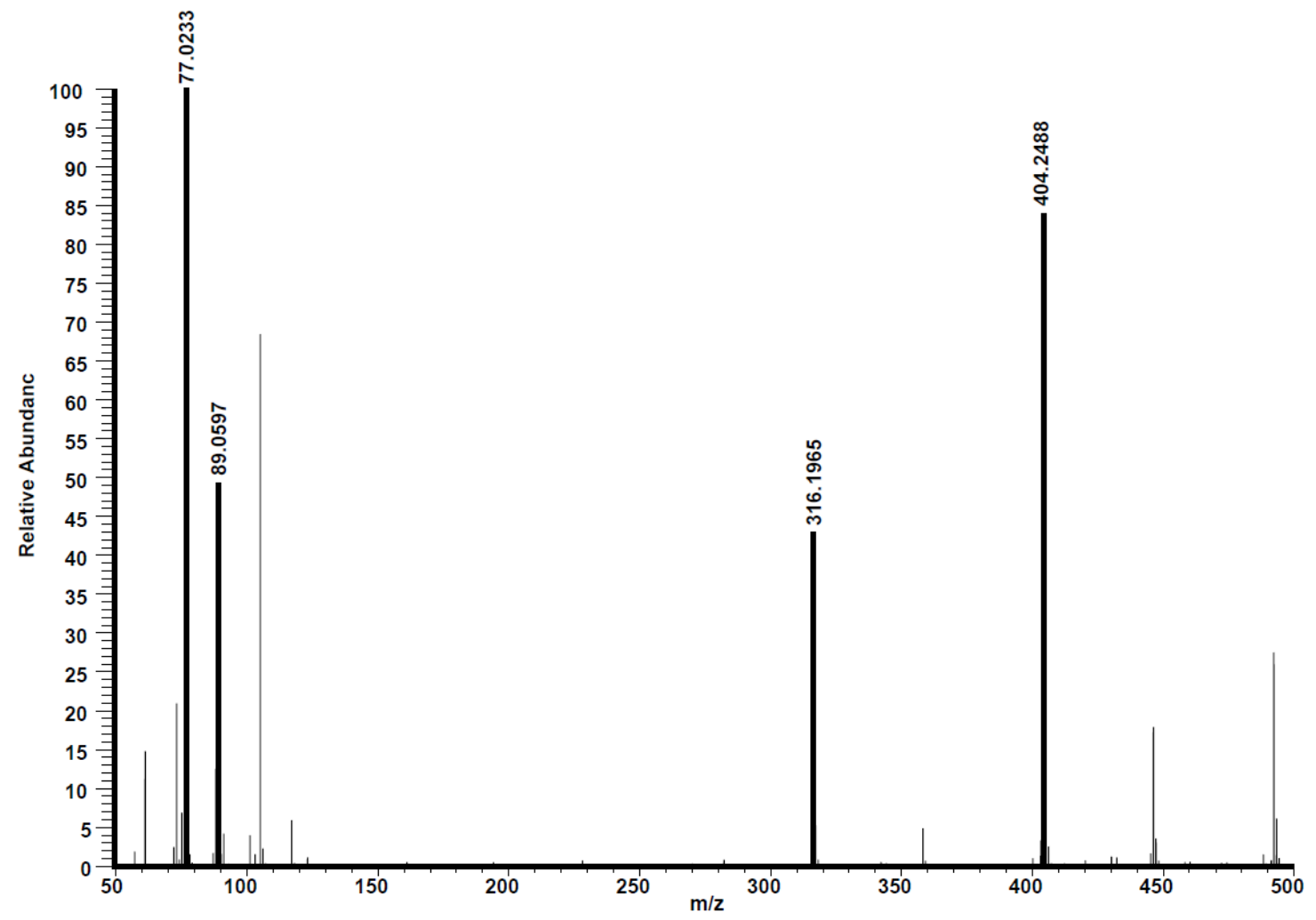

Figure 32. Positive mode, full scan high-resolution mass spectrum for MEKP analyzed from the crude synthesized product. Ions characteristic of MEKP have been bolded and labelled. 


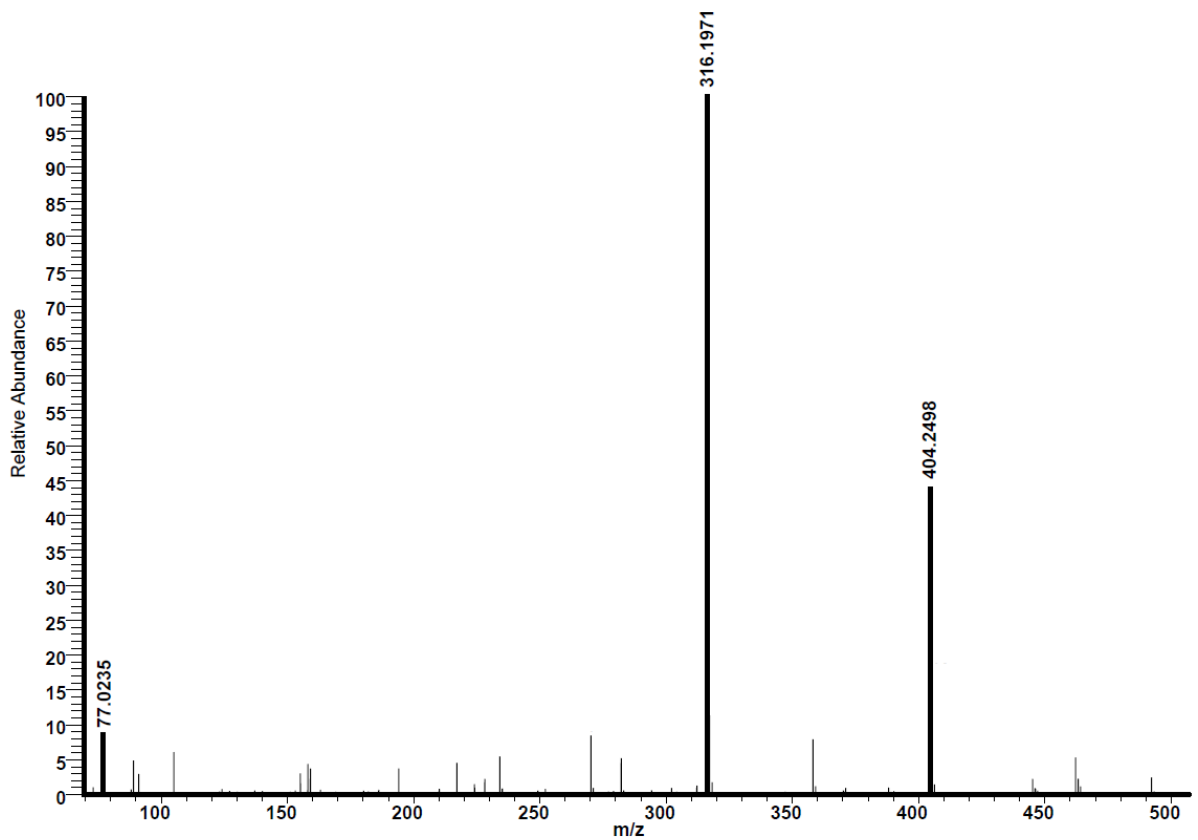

Figure 33. Positive mode, full scan high-resolution mass spectrum depicting identification of MEKP from direct analysis of fragment collected post-blast from IED \#5. lons characteristic of MEKP have been bolded and labelled.

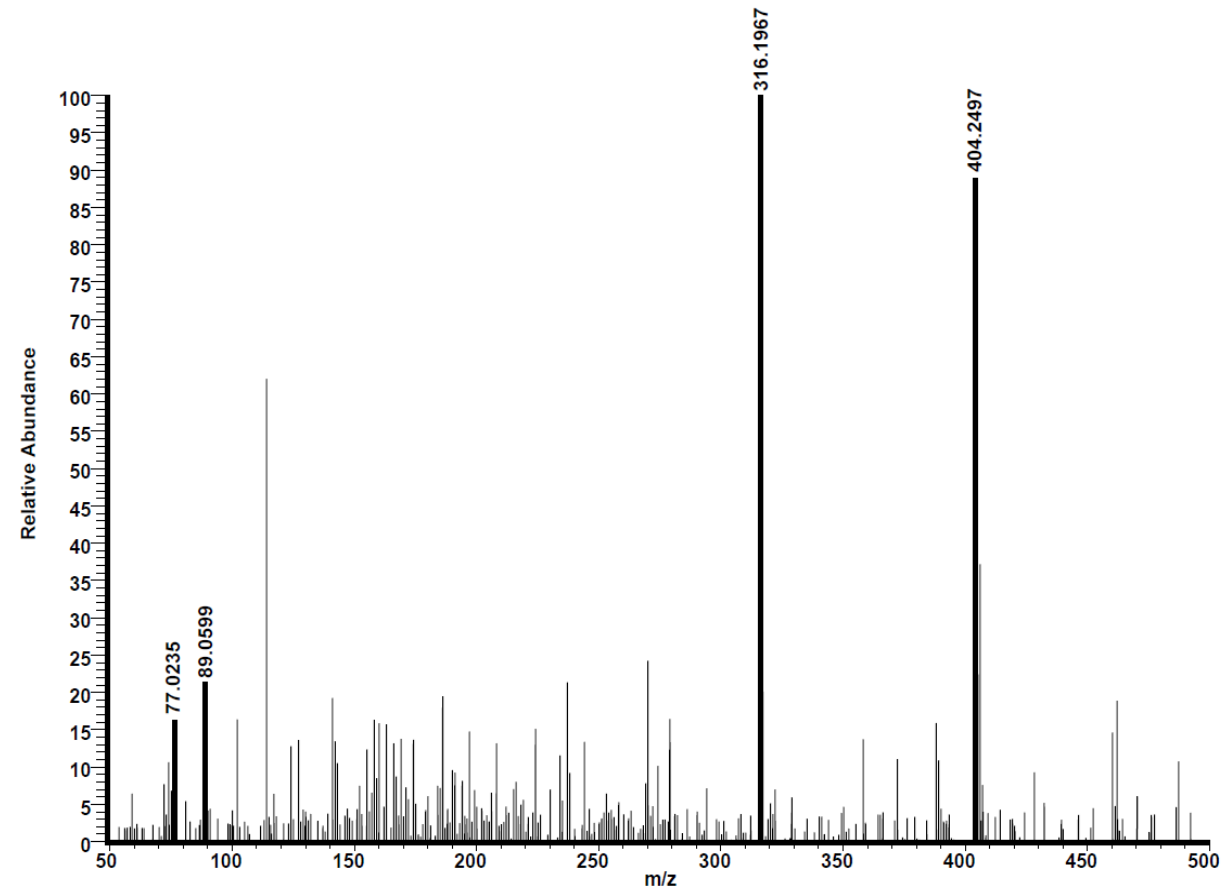

Figure 34. Positive mode, full scan high-resolution mass spectrum depicting identification of MEKP upon analysis of a swab used to collected post-blast residues from IED \#5 fragments. Ions characteristic of MEKP have been bolded and labelled. 
Both direct and in-direct analysis of the post-blast fragments permitted identification of each OPBE used. In-direct analysis using swabbing provided a mechanism to sample large or irregular objects not amenable to direct analysis. Swabbing also enhances recovery by accumulating more residue on a smaller surface area. Therefore, further optimization was completed to determine the most suitable swab substrate and compare recovery with dry and solvent-dampened swabs.

Post-blast fragments from IED \#3 were used for this optimization work. Swabs made of cotton, Teflon and paper modified with a thin adhesive coating were tested to determine their respective recovery efficiencies (Figure 35). Cotton swabs are reported to be an effective resource for collecting samples of forensic interests. ${ }^{65}$ Collection of residues using cotton swabs resulted in positive identification of the explosive used (HMTD) (Table 5) and cotton was therefore chosen as the preferred swab material. Detection of explosives present on a swab via analysis in transmission mode requires the active ionizing gas stream to pass through the material and interact with the residues present. Compared to the modified paper swabs, cotton swabs are sufficiently porous to facilitate desorption and ionization for MS detection. Using cotton swabs is in agreement with best practice for forensic sample collection as cotton swabs are regularly recommended to front-line staff members and investigators for sample collection 
Table 5. Characteristic ions of HMTD present upon analysis of residues collected via swabs differentiated by substrate.

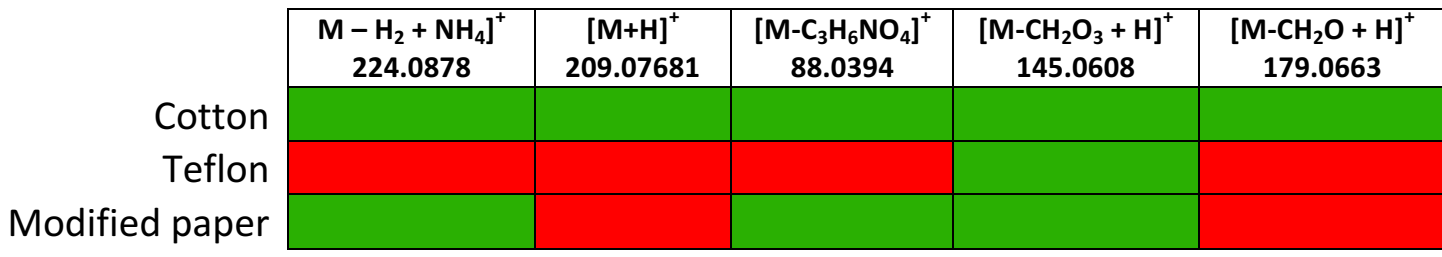
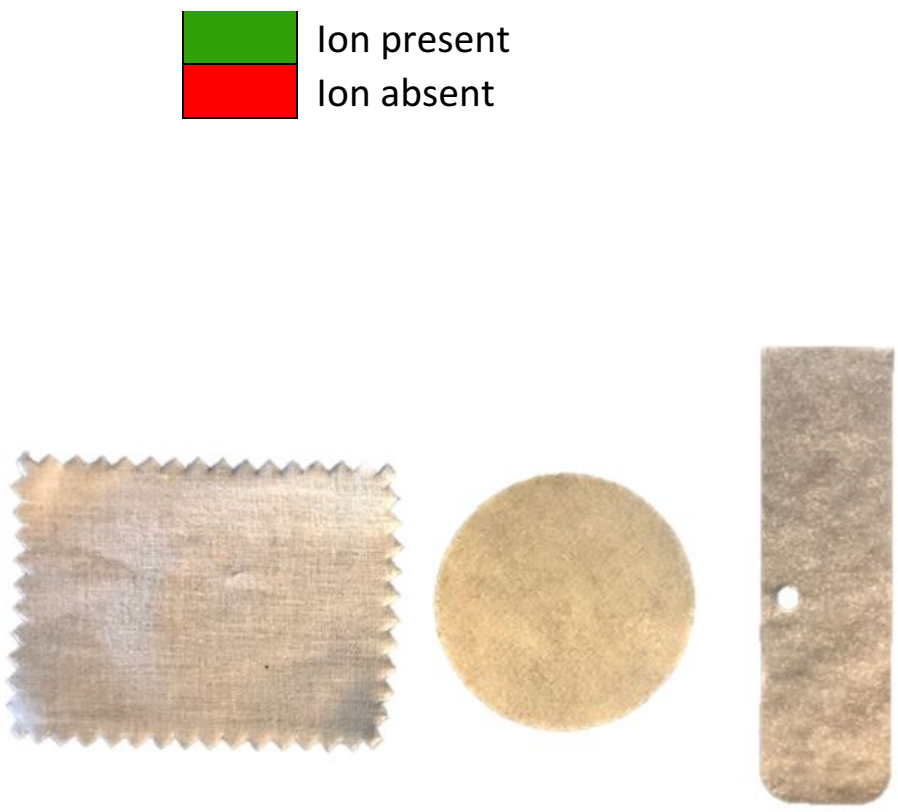

Figure 35. From left to right - cotton swab, paper swab, modified paper swab.

Analysis of dry versus wet swabs was conducted to evaluate any difference in effective recovery of residues when using (or not using) solvents. Analysis of dry swabs collected from, afforded identification of HMTD (top, Figure 36). DART-MS analysis of swabs wetted with acetone, acetonitrile or methanol resulted in positive identification of HMTD from IED\#5 container (Figure 36). Wetting a swab with a solvent prior to swabbing can enhance explosive residue recovery ${ }^{9}$, but solvents will also co-dissolve other substances present from environmental contamination or the substrate itself. Clean-up procedures for swab extracts prior to analysis can be used. ${ }^{9}$ Dry swabbing, however, will 
not dissolve any substances or the substrate, reducing unwanted sample matrix and fewer background ions to clutter the MS spectrum (Figure 36). Dry swabbing can eliminate extraction or clean-up, and was found effective for explosive residue recovery. When analyzing dry and wet swabs collected from the post-blast container of IED \#3, we observed an additional predominant ion m/z 207.0614 (Figure 35) not apparent from direct analysis (Figure 29). This daughter ion is best calculated as $\left[\mathrm{C}_{6} \mathrm{H}_{11} \mathrm{~N}_{2} \mathrm{O}_{6}\right]^{+}$which is in agreement with structural assignment as the dialdehyde derivative protonated instead of ammoniated, i.e. $\left[\mathrm{M}-\mathrm{H}_{2}+\mathrm{H}\right]^{+}$. The appearance of ion $\mathrm{m} / \mathrm{z} 207.0614$ post-blast but not apparent from the reference material can be associated with degradation from blast effects (heat, pressure). To the best of our knowledge, ion $\mathrm{m} / \mathrm{z} 207.0614$ has not been previously reported from previous LCMS studies. ${ }^{10,62}$ 

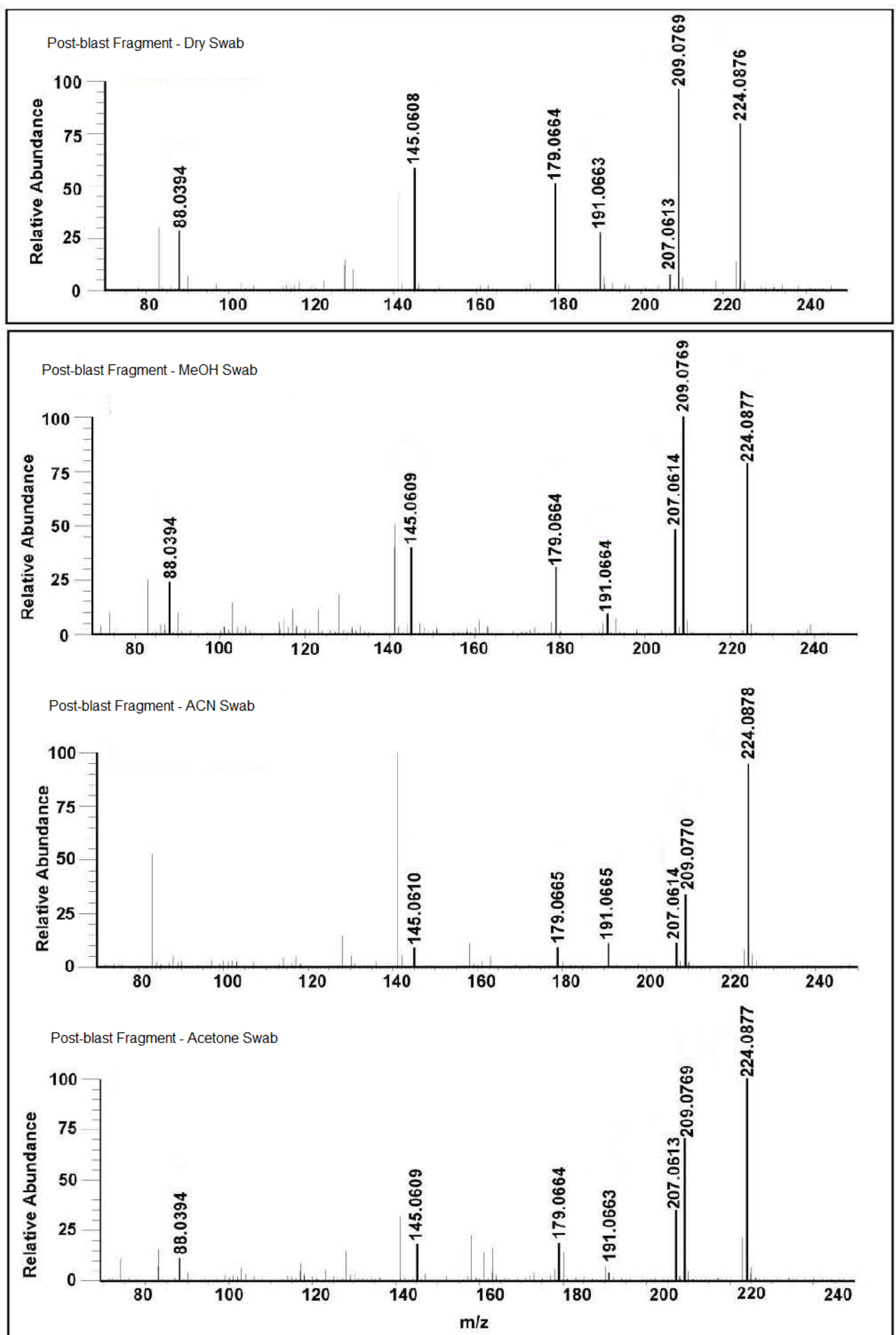

Figure 36. Full scan high-resolution mass spectra depicting identification of HMTD upon collection of post-blast residues from IED \#5 using dry and solvent dampened swabs. 


\subsubsection{Binary Explosives}

Homemade binary explosives were included in this study because the individual components (i.e. fuel and oxidizer) can be easily sourced from common commercial products and pose a significant threat for fabricating IEDs. Detection of binary explosives products post-blast poses challenges because the different chemical nature between fuels and oxidizers necessitates different analytical tools. Many of the commercial products containing the precursor chemicals have complex matrices arising from the addition of different stabilizers, fragrances and preservatives. Using DART-MS, the fuel and oxidizer can be detected separately by switching between positive or negative polarity. Examples of common commercial sources of HME precursor fuels and oxidizers are listed in Table 1.

Currently, the RCMP does not have a valid method for characterization of sugarbased fuels post-blast. The use of DART-MS to detect sugar-based fuels was thus explored as a potential solution to this capability gap. A variety of different commercially available carbohydrate-based or sugar-containing products are suitable as fuel sources, making identification of any source challenging. The main charge of IEDs \#11 and 12 was binary explosive prepared using a carbohydrate-based fuels (TANG ${ }^{\circledR}$ juice mix and dextrin). TANG $^{\circledR}$ is a food-grade source of sucrose, which is the main ingredient. Sucrose is the dimer glucose and fructose monomers linked via $\alpha(1,2)$ glycosidic linkages. Dextrin is a complex carbohydrate polymer composed of highly cross-linked glucose units. DART-MS analysis of sucrose, TANG and dextrin in dilute solutions verified each carbohydrate fuel was identifiable (Figures 38, 39 and 43). Because glucose is a base monomer in either carbohydrate, analysis of either sucrose or dextrin resulted in a similar MS spectrum and 
fragmentation pattern, including common ions that can be used to identify each fuel when compared to a glucose reference material. ${ }^{61}$ DART-MS analysis of glucose yields the following characteristic ions (Figure 37); ammoniated glucose ion (m/z 198.0973 $\left.\left[\mathrm{C}_{6} \mathrm{H}_{12} \mathrm{O}_{6}+\mathrm{NH}_{4}\right]^{+}\right)$and fragment ions via the loss of water $\left(\mathrm{m} / \mathrm{z} 180.0867\left[\mathrm{C}_{6} \mathrm{H}_{12} \mathrm{O}_{6}+\mathrm{NH}_{4}-\right.\right.$ $\left.\mathrm{H}_{2} \mathrm{O}\right]^{+}, \mathrm{m} / \mathrm{z} 163.0601\left[\mathrm{C}_{6} \mathrm{H}_{12} \mathrm{O}_{6}+\mathrm{NH}_{4}-\mathrm{NH}_{3}-\mathrm{H}_{2} \mathrm{O}\right]^{+}, \mathrm{m} / \mathrm{z} 145.0496\left[\mathrm{C}_{6} \mathrm{H}_{12} \mathrm{O}_{6}+\mathrm{NH}_{4}-\mathrm{NH}_{3}-\right.$ $\left.2 \mathrm{H}_{2} \mathrm{O}\right]^{+}$and $\left.\mathrm{m} / \mathrm{z} 123.0390\left[\mathrm{C}_{6} \mathrm{H}_{12} \mathrm{O}_{6}+\mathrm{NH}_{4}-\mathrm{NH}_{3}-3 \mathrm{H}_{2} \mathrm{O}\right]^{+}\right) .{ }^{60}$ Characteristic ions were detected with an absolute abundance of $10^{5}-10^{6}$ and spectral background ions remained between $10^{3}-10^{4}$ counts.

A blended product of a sugar-derived fuel and a strong oxidizer is an example of high-brisance explosive. Consequently, many of the IED fragments recovered post-blast were small (e.g. SIM card $\sim 0.25 \mathrm{~cm}^{2}$ ) rendering recovery of residue for detection by DARTMS difficult. Co-extraction of multiple fragments of similar materials together afforded detection of a carbohydrate based fuel in IED \#11 and 12 via DART-MS analysis (Figures 40-42 and 44-46). This is a common sampling method used in the forensic analysis of postblast explosives. Similar DART-MS spectrum of both sucrose and dextrin prevents identification of the commercial product source post-blast. Because most carbohydratebased fuels are non-volatile residue loss by evaporation is not a concern. Sugar-derived fuel residues are expected to persist and be detectable if analyzed at a later time, which is analogous with the expectation for the inorganic residues of the oxidizer salts used in the same binary explosive mixtures. 


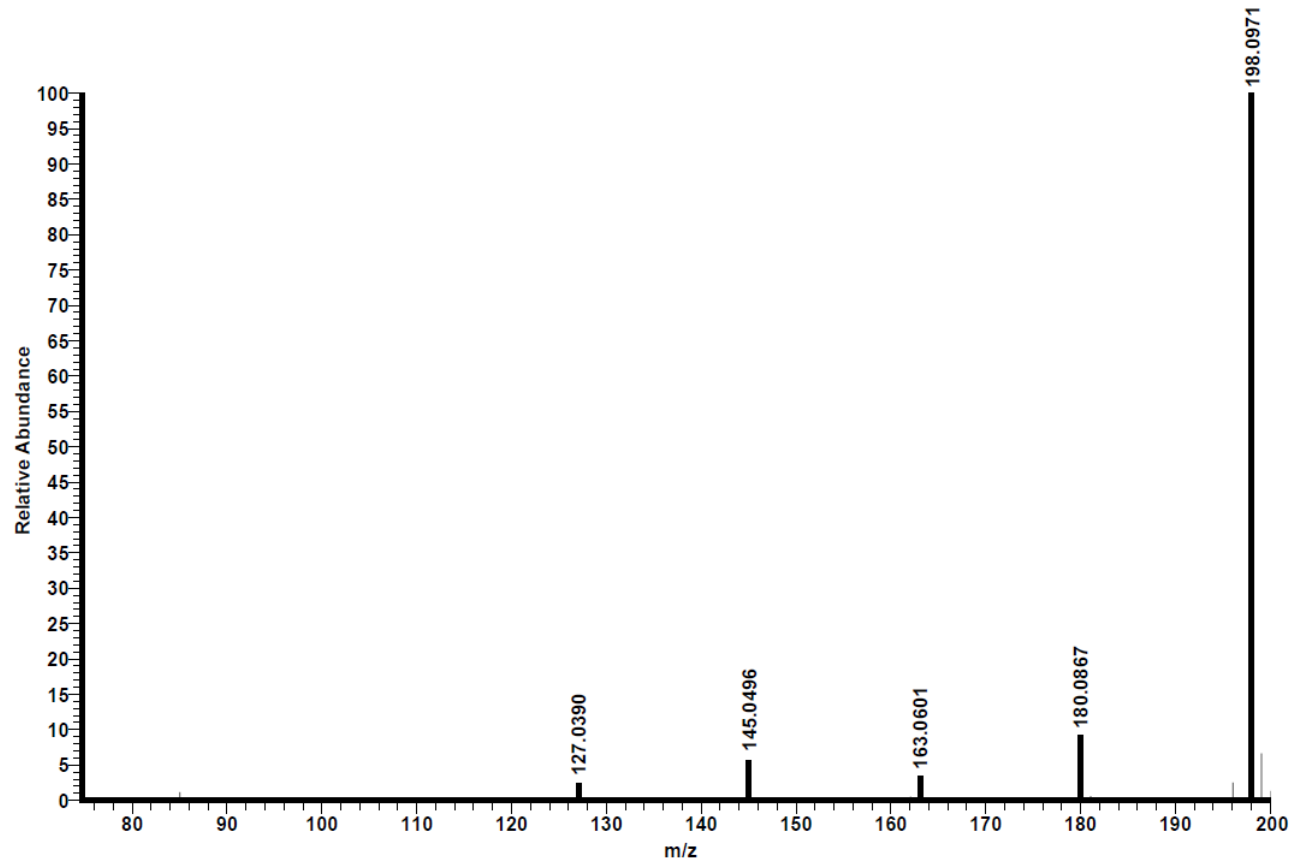

Figure 37. Full scan high-resolution mass spectrum of glucose dissolved in water as a reference material. Collected in positive mode.

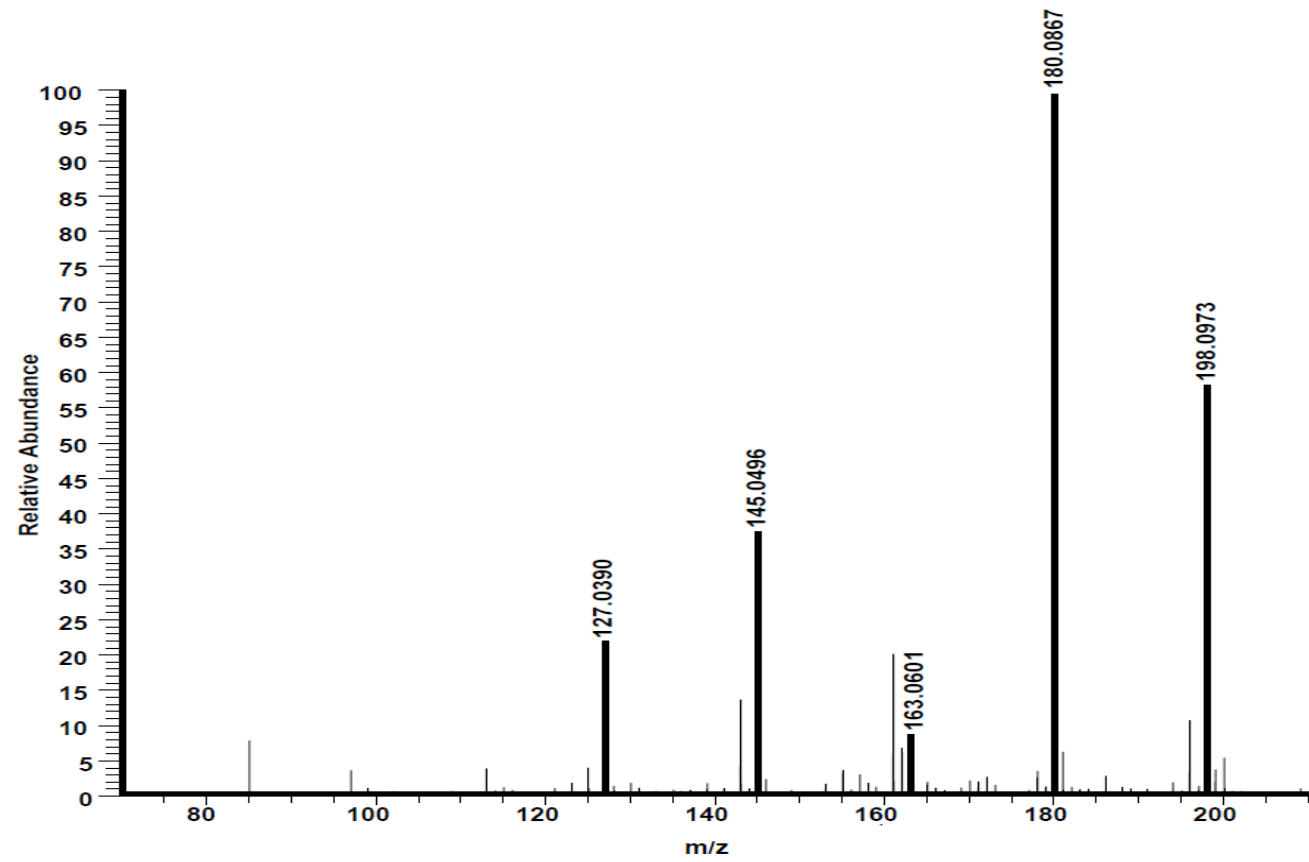

Figure 38. Full scan high- resolution mass spectrum of sucrose dissolved in water, used as a reference material. Collected in positive mode. 


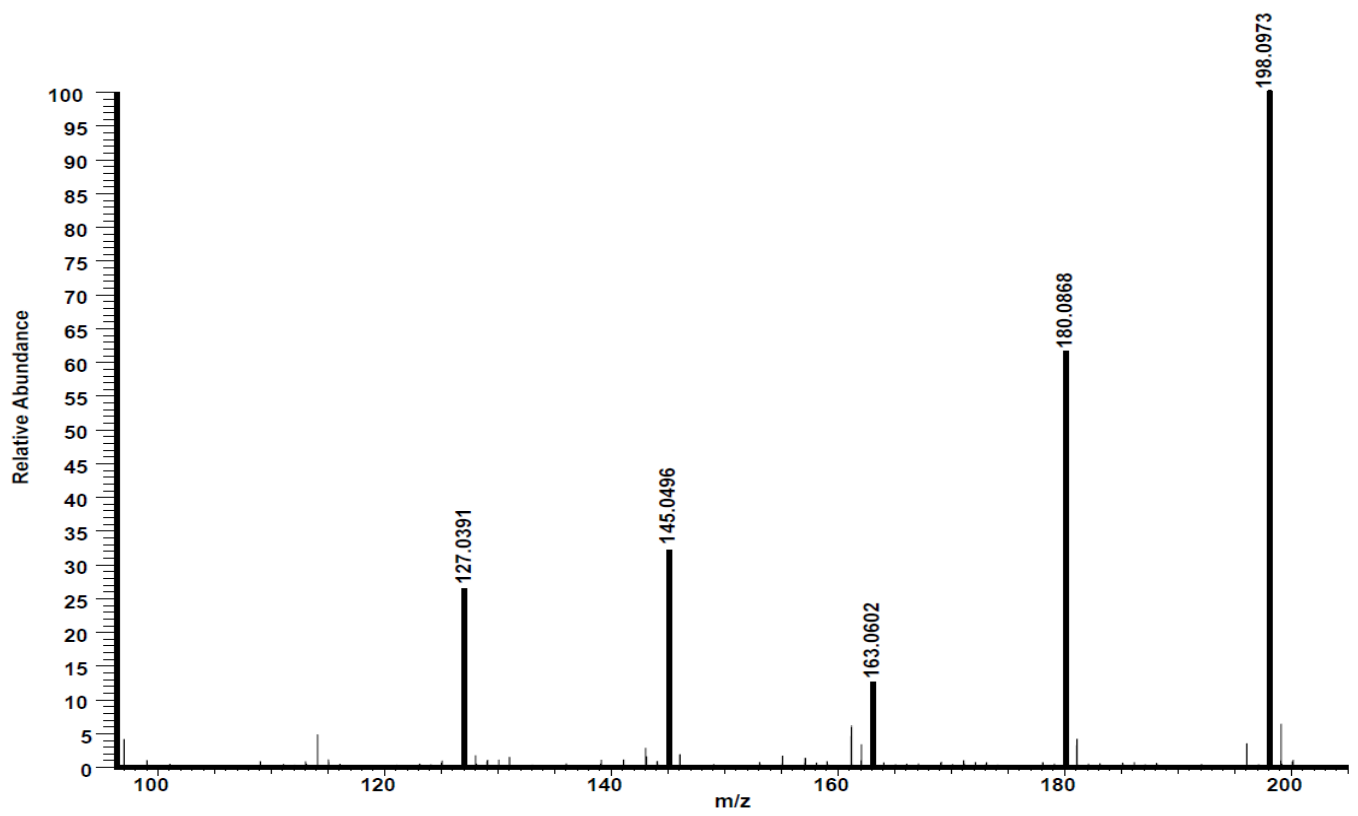

Figure 39. Full scan high-resolution mass spectrum of TANG dissolved in water, used as a reference material. Collected in positive mode.

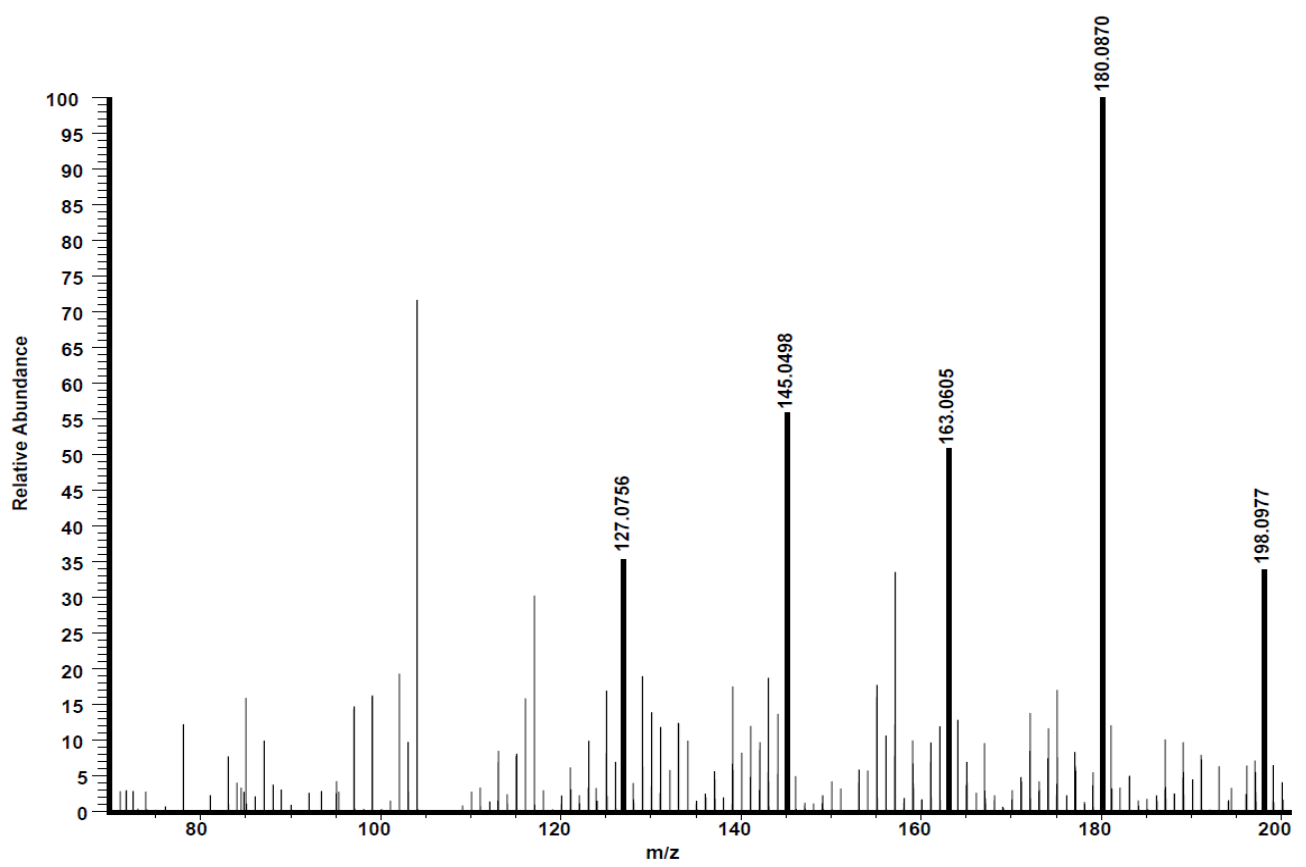

Figure 40. Full scan high-resolution mass spectrum depicting identification of a glucose containing product from post-blast residues extracted with water from IED \#11 metal substrate fragment. Collected in positive mode. 


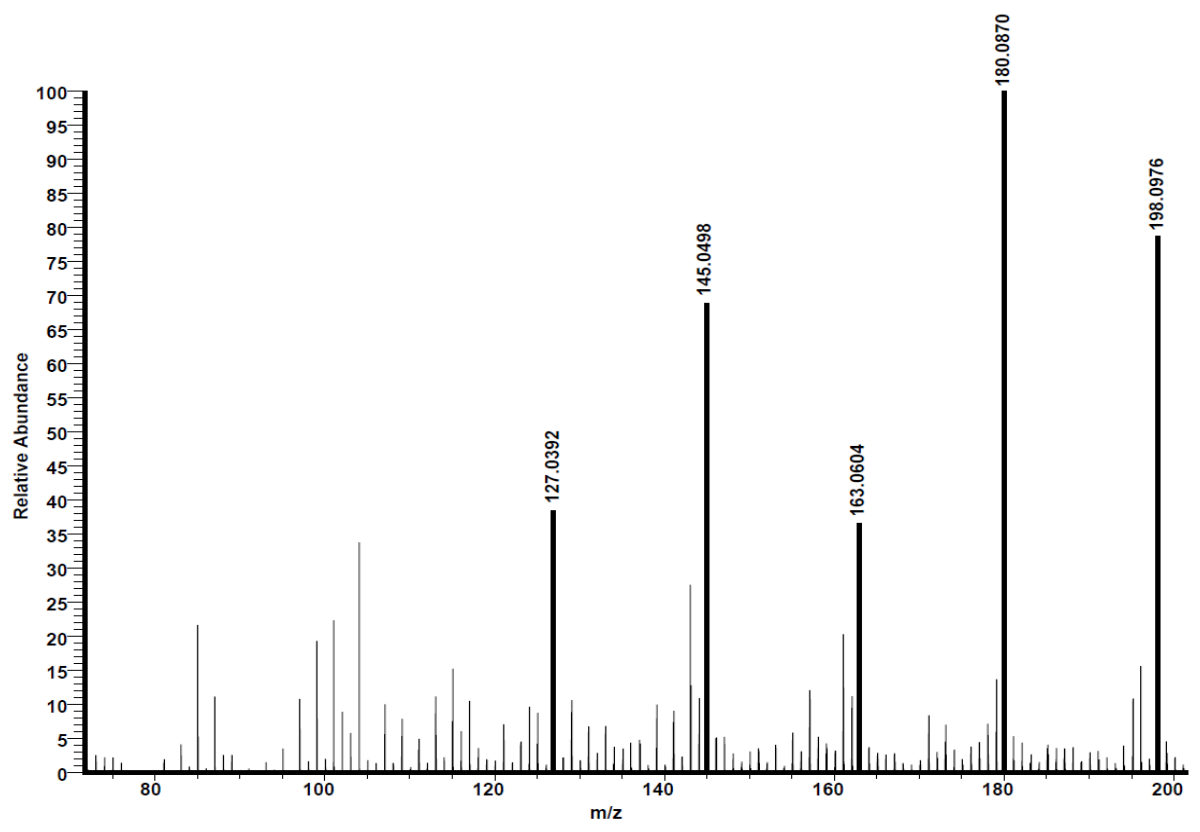

Figure 41. Full scan high-resolution mass spectrum depicting identification of a glucose containing product from post-blast residues extracted with water from IED \#11 plastic substrate fragment. Collected in positive mode.

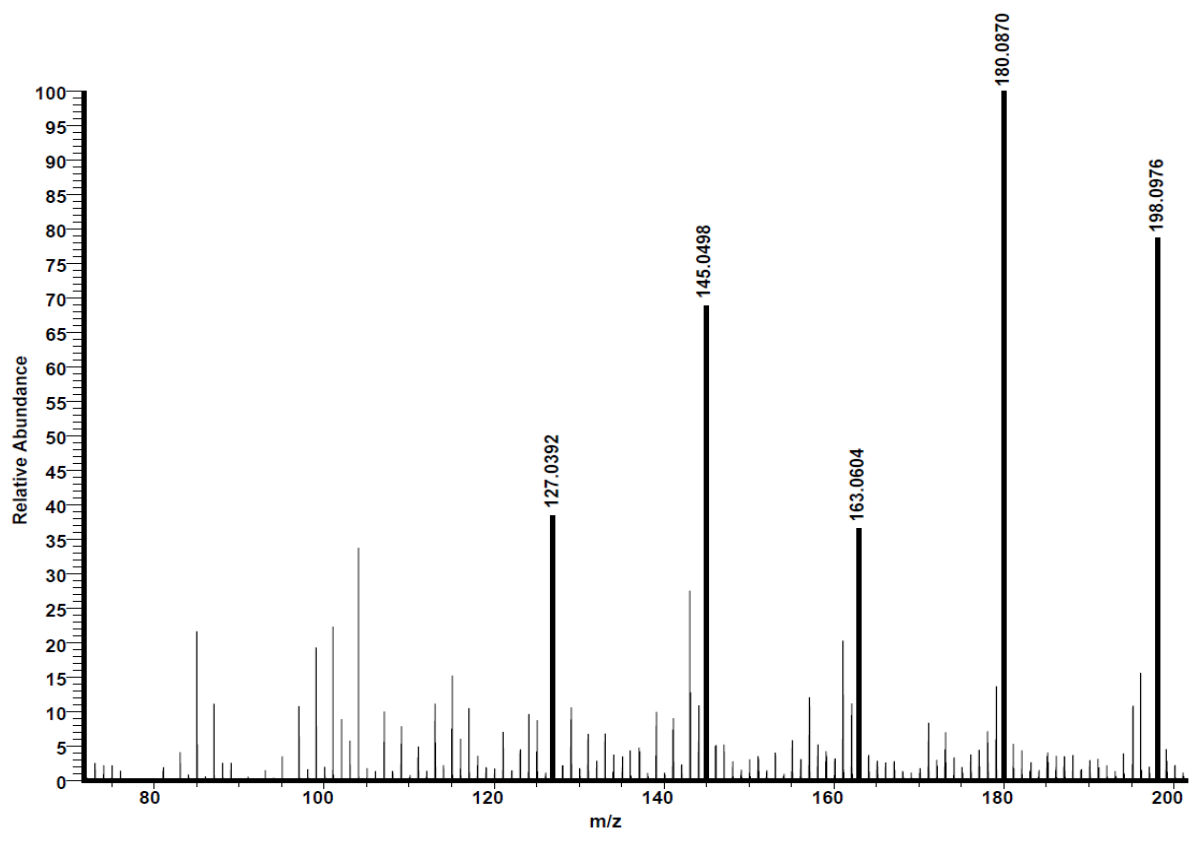

Figure 42. Full scan high-resolution mass spectrum depicting identification of a glucose containing product from post-blast residues extracted with water from IED \#11 rubber substrate fragment. Collected in positive mode. 


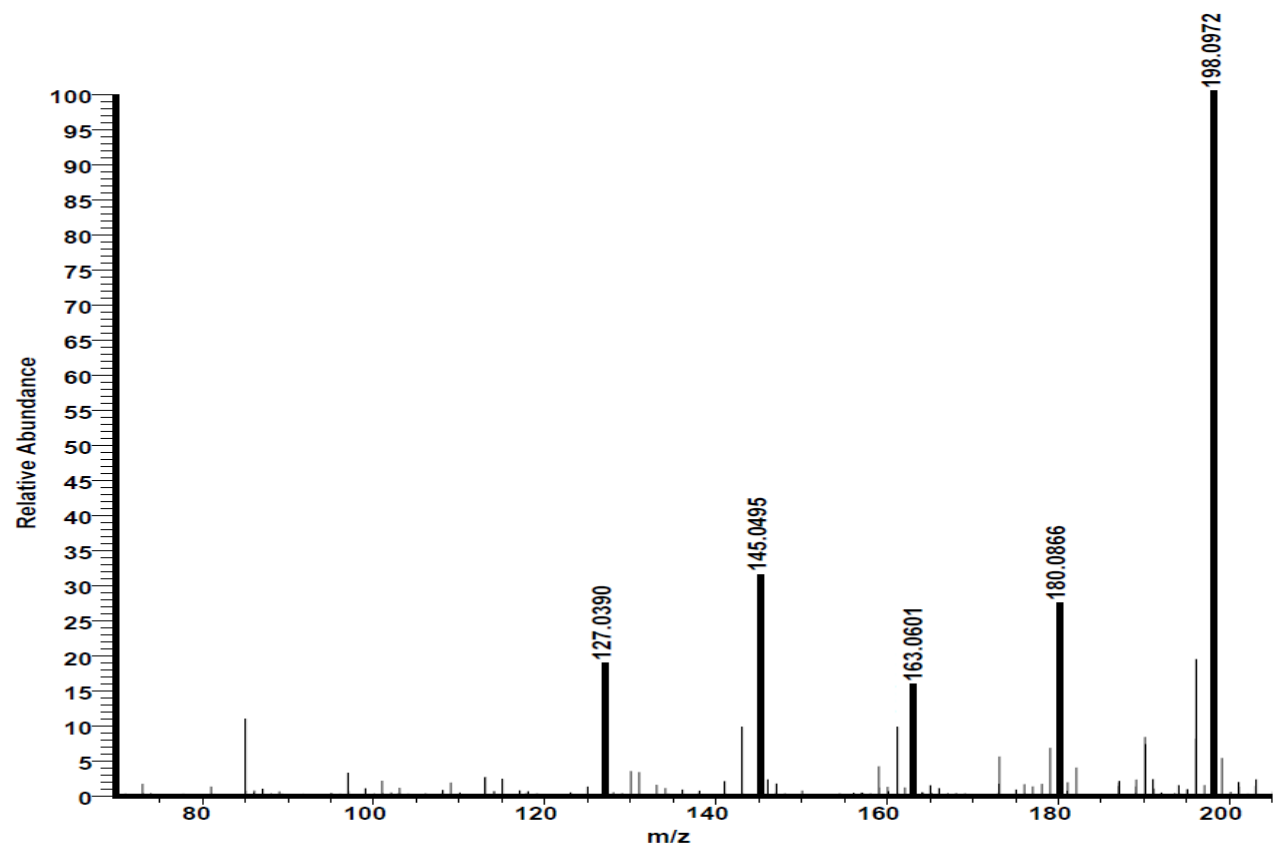

Figure 43. Full scan high- resolution mass spectrum of dextrin reference material dissolved in water. Collected in positive mode.

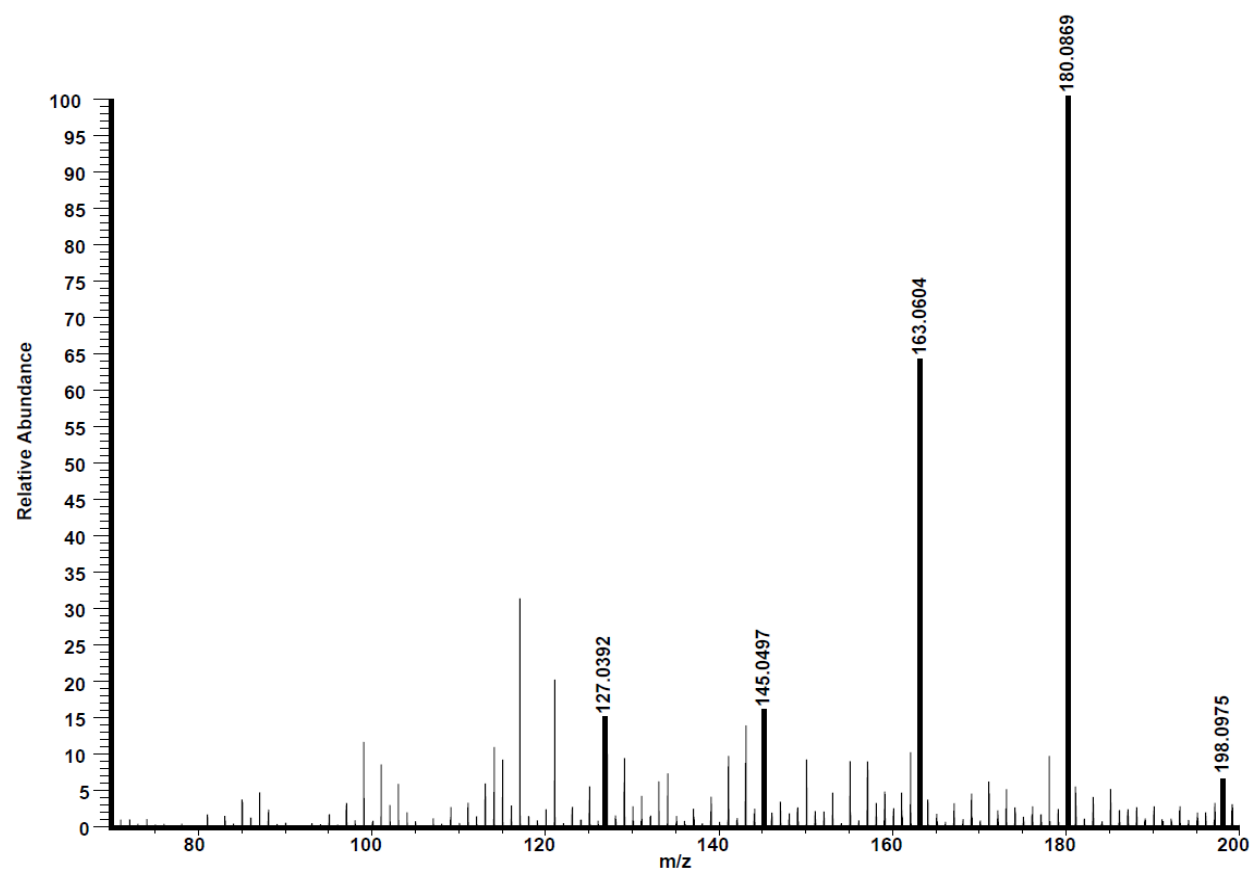

Figure 44. Full scan high-resolution mass spectrum depicting identification of a glucose containing product from post-blast residues extracted with water from IED \#12 plastic substrate fragment. Collected in positive mode. 


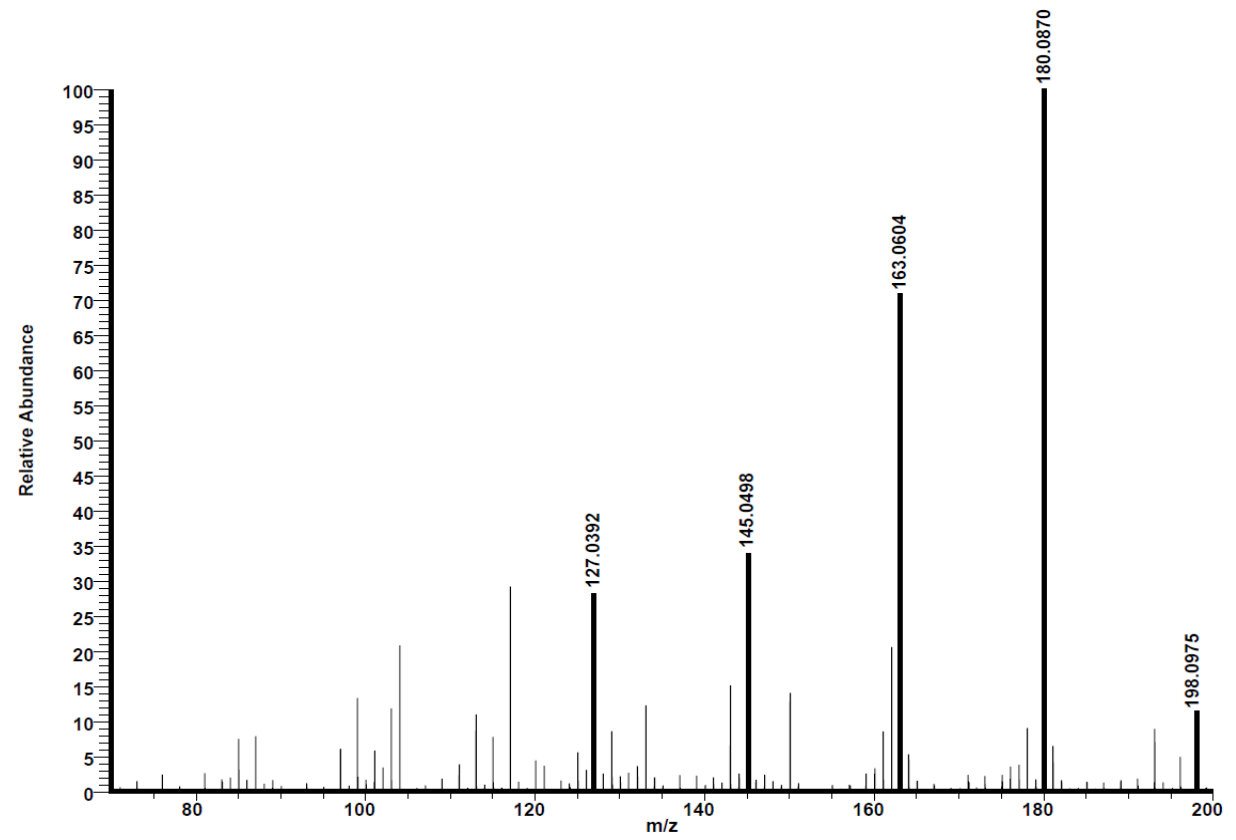

Figure 45. Full scan high-resolution mass spectrum depicting identification of a glucose containing product from post-blast residues extracted with water from IED \#12 metal substrate fragment. Collected in positive mode.

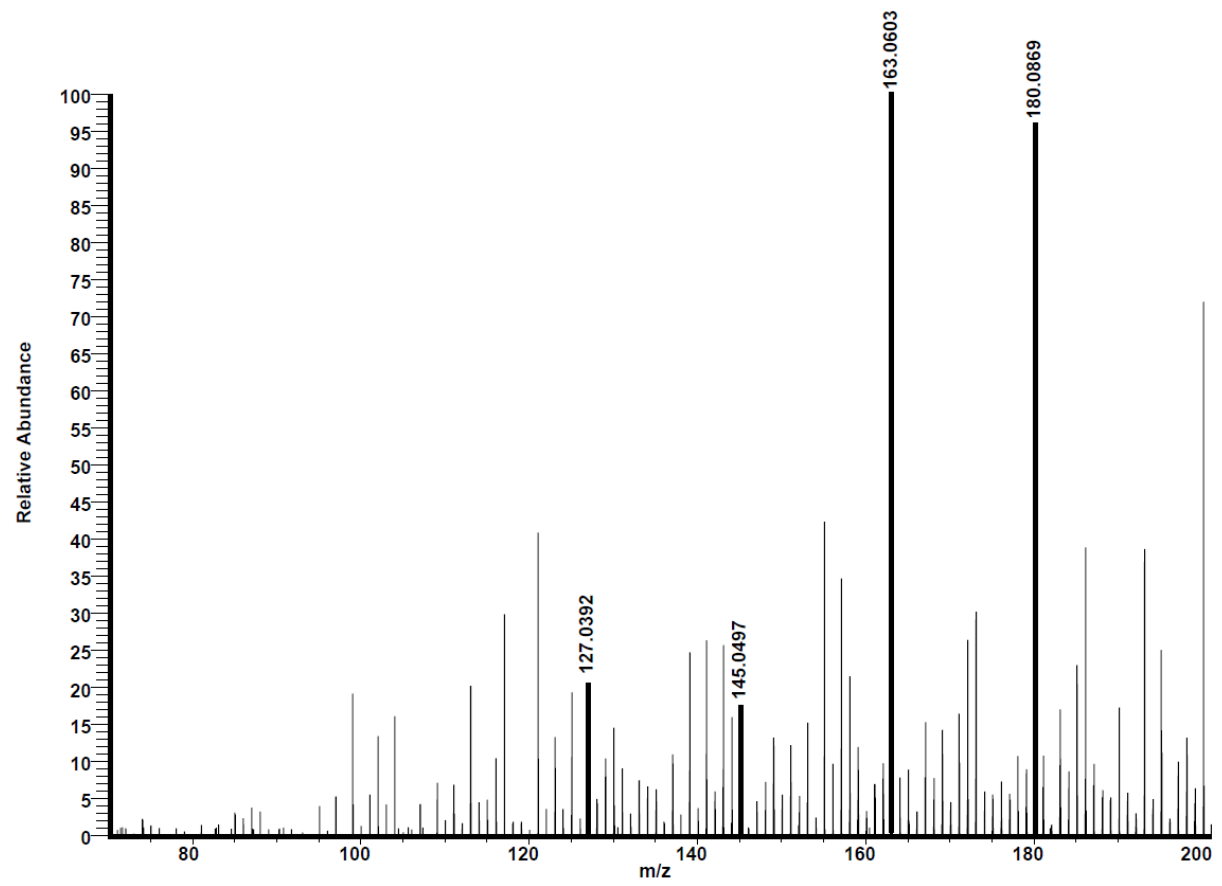

Figure 46. Full scan high-resolution mass spectrum depicting identification of a glucose containing product from post-blast residues extracted with water from IED \#12 rubber substrate fragment. Collected in positive mode. 
Currently, GC-MS is the quality assured method used by the RCMP for the analysis of petroleum-derived fuels in binary explosives. However, DART-MS provides a suitable and rapid alternative. A variety of common petroleum-derived products encountered in binary explosives were surveyed (Table 1 ) and each fuel exhibits a different, distinctive profile (Figures 47, 60-63). It was beyond the scope of this work to analyze all the different types of petroleum-derived fuels that could potentially be used to make a binary HME. Each fuel analyzed was diluted in hexane, which is a solvent commonly used to extract petroleum-derived fuels from explosives. Analysis of neat hexane assisted in the background subtraction of any solvent associated ions (Figure 25). Identification of automotive grease post-blast was investigated by analyzing fragments from IED \#10.

Automotive greases are manufactured by blending many different substances together (e.g. lubricants, thickener, additives, preservatives), which together affords a complex mixture. Analysis of a typical automotive lubricating grease (Figure 47) exhibited a high-abundance pattern of low mass ions in the $\mathrm{m} / \mathrm{z}$ range of $100-250$ and a lowerabundance hydrocarbon profile in the $\mathrm{m} / \mathrm{z}$ range of $300-450$. Analysis of hexane extracts from the post-blast fragments (e.g. metal, plastic and rubber substrate fragments) resulted in observation of three predominant ions $(\mathrm{m} / \mathrm{z} 149.0237,279.1589$, and 391.2844) (Figure 48-50). EICs depicted observation of these ions in the DART-MS spectrum of the unused automotive grease, which was analyzed as a reference material. However, the ions observed post-blast were not as abundantly observed for the unused grease. This is not unexpected, as the blast effects (heat, pressure) are known to change the composition of petroleum-derived fuels, as is commonly observed in ignitable liquid 
analysis. ${ }^{63}$ The best practice to identify the combustion residue of a petroleum-derived fuel is to compare with a suitably weathered or ignited reference material; the scope of which was beyond this present study. Regardless, the detection of ions post-blast that are identifiably common with the unused grease indicates that a petroleum-derived grease fuel can be identified post-blast.

Combustion is expected to consume some (or all) of the components of the grease. Pyrolysis and other irreversible oxidation side-reactions are also expected to occur during the explosion. ${ }^{66}$ Consequently, a weathered sample of grease is expected to afford a more representative material for comparison and identification of a grease postblast, which is the common technique used in fire-debris interpretation of ignitable liquids after an arson fire. ${ }^{66}$ Weathering grease samples is not a straightforward task. The preparation of suitably weathered petroleum-derived fuels as reference samples for comparison with residue analysis of fuel-products used in devices \#6-10 by DART-MS will be the subject of future work. 


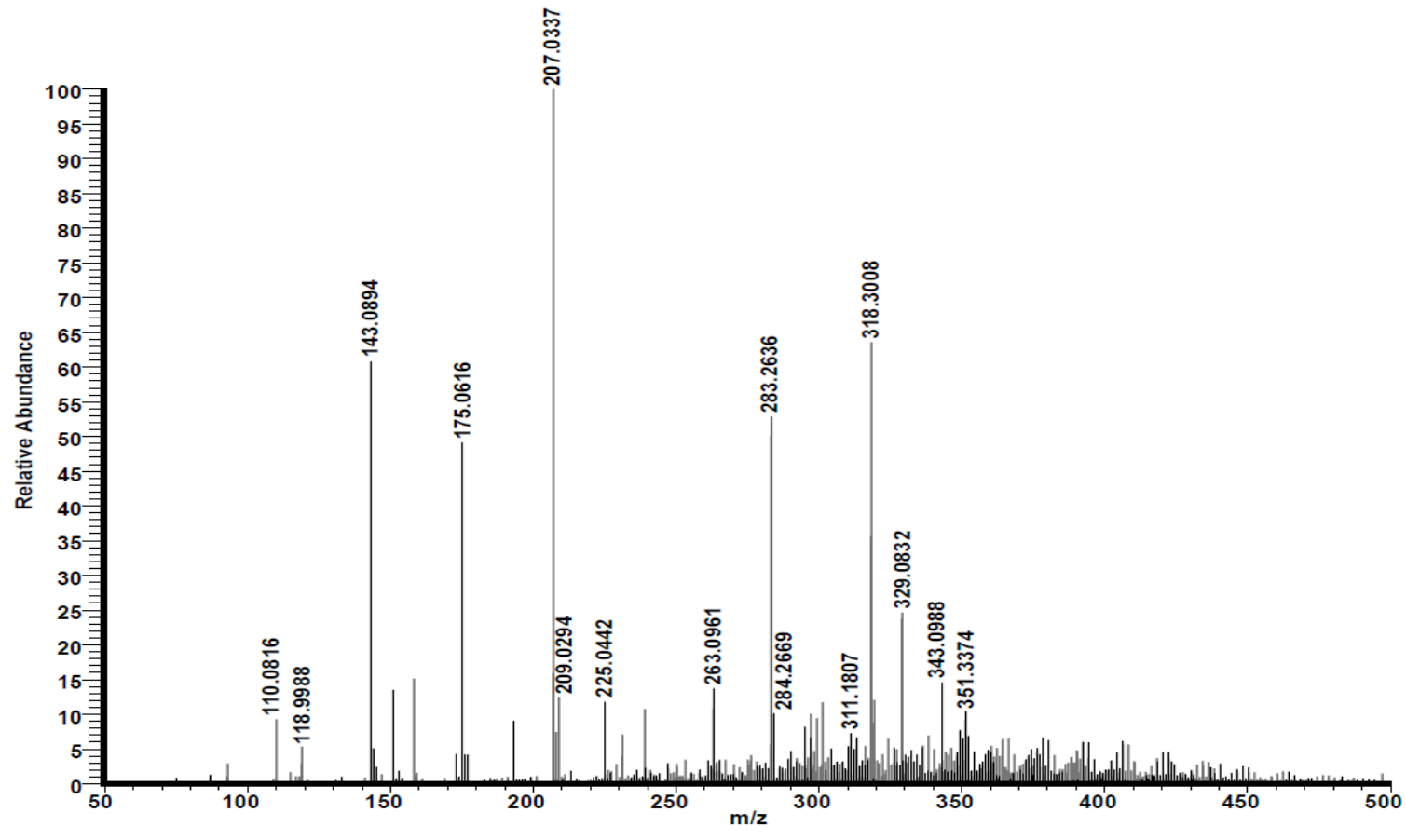

Figure 47 Full scan high-resolution mass spectrum of an automotive grease, used as a reference material. Collected in positive mode.

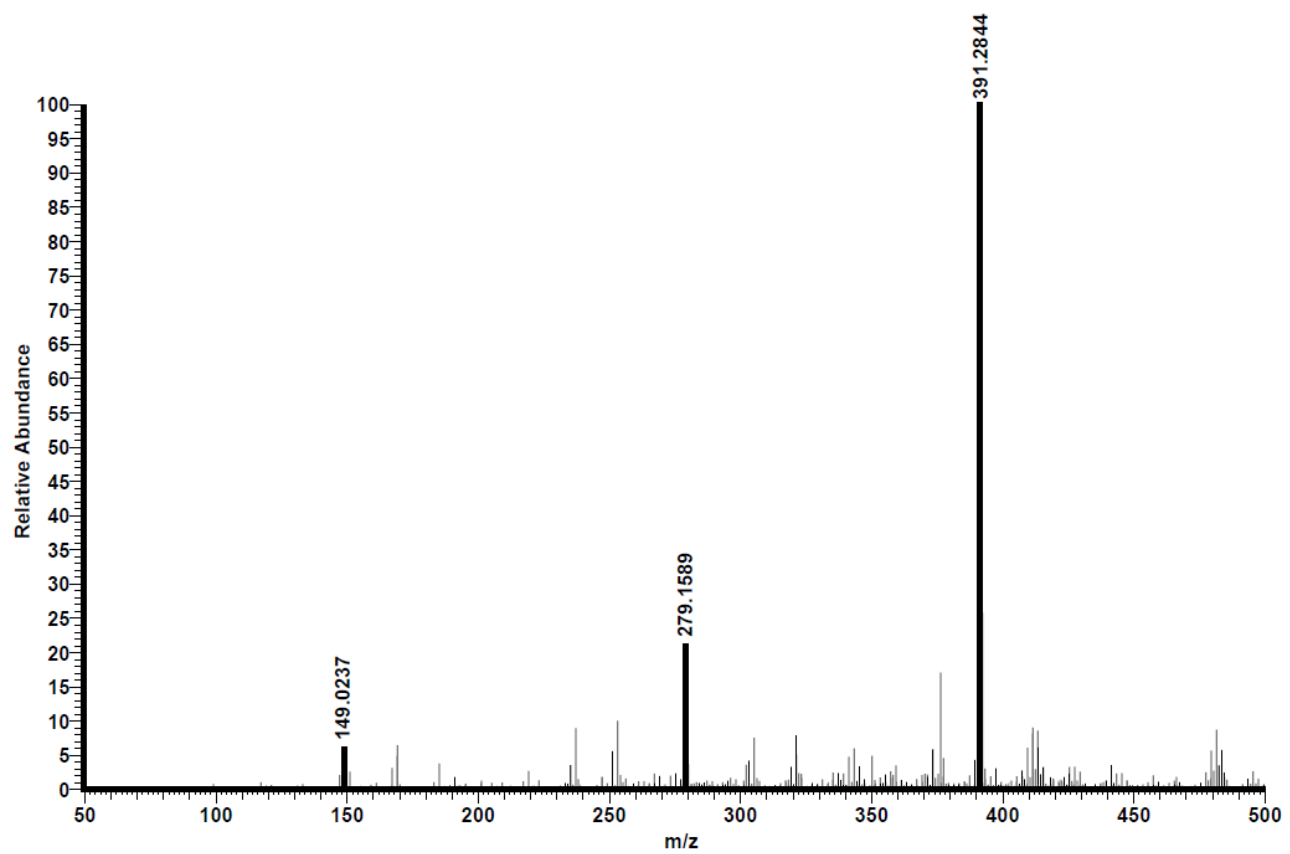

Figure 48. Full scan high-resolution mass spectrum depicting identification of automotive grease from post-blast residues extracted with hexane from IED \#10 metal substrate fragment. Collected in positive mode. 


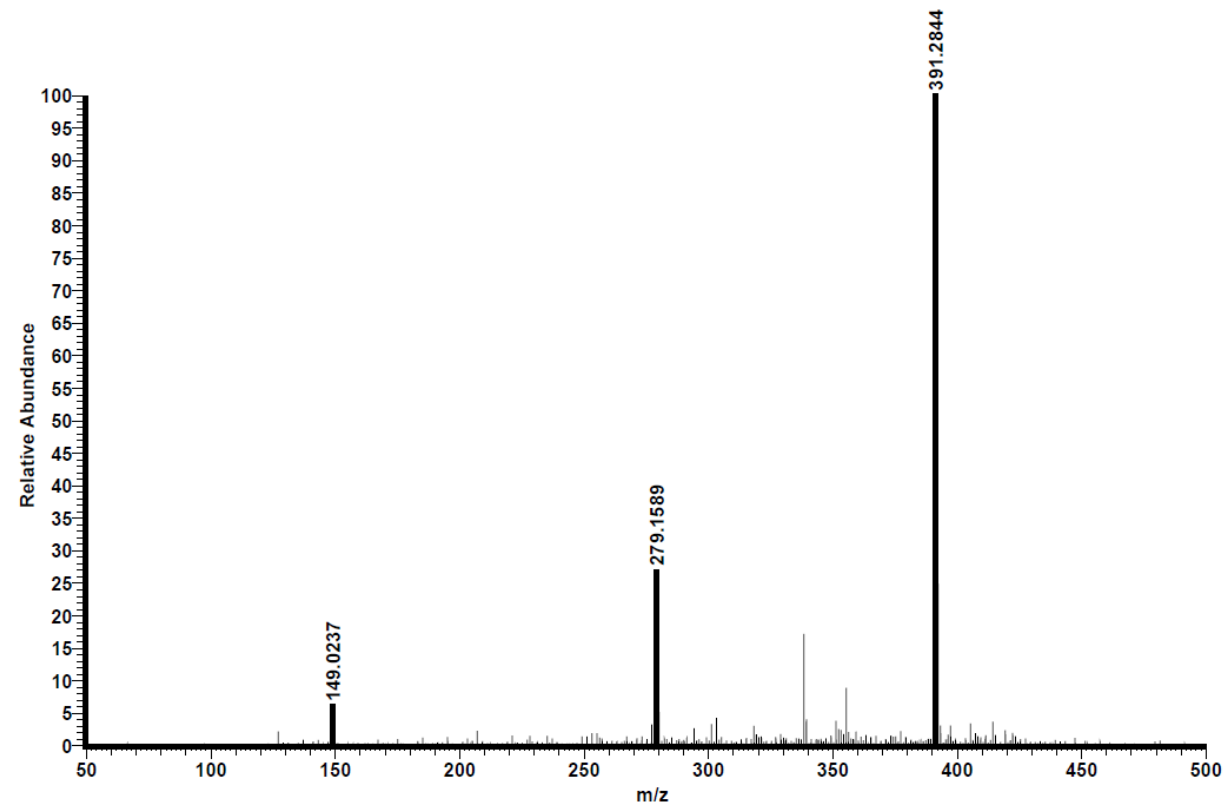

Figure 49. Full scan high-resolution mass spectrum depicting identification of automotive grease from post-blast residues extracted with hexane from IED \#10 plastic substrate fragment. Collected in positive mode.

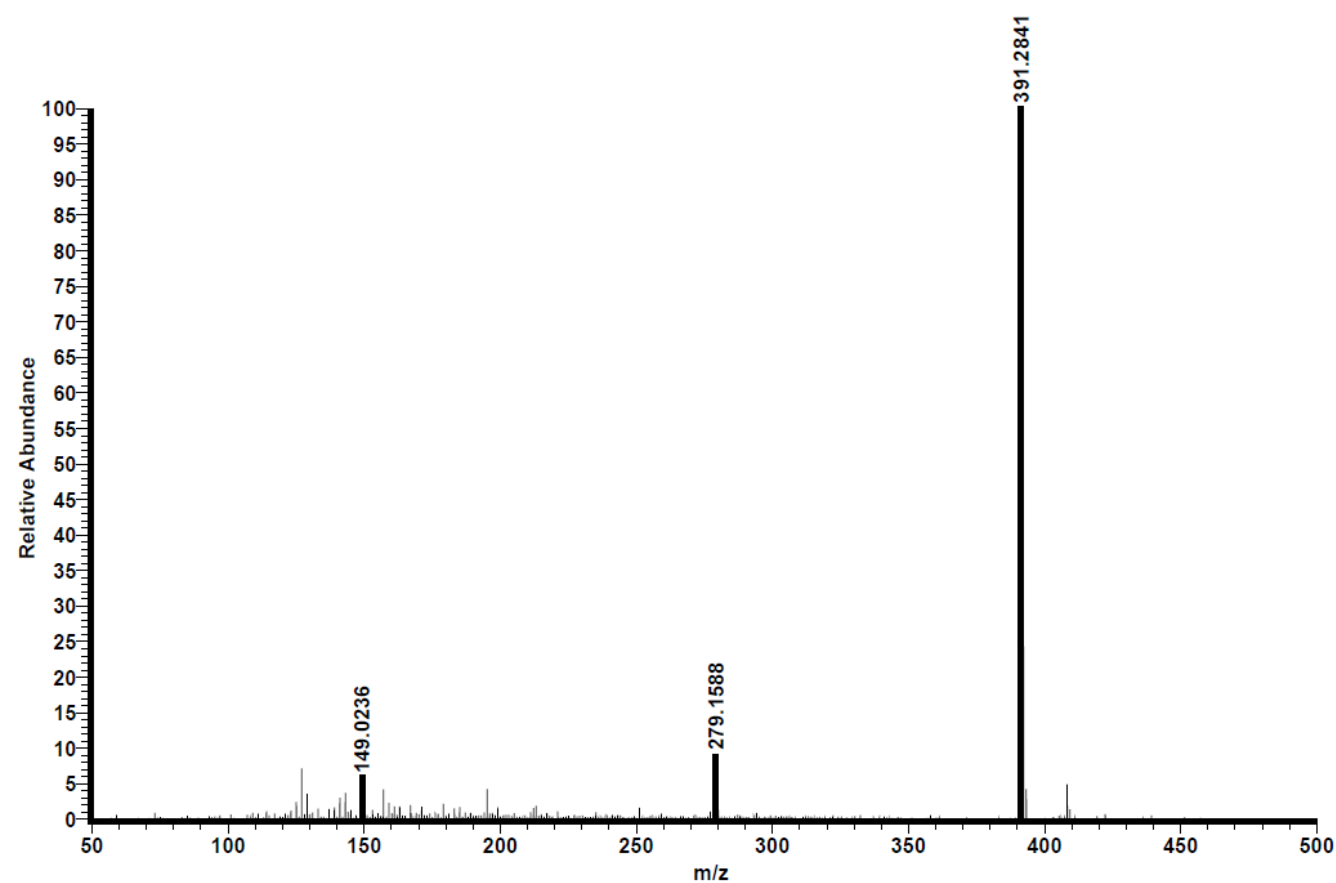

Figure 50. Full scan high-resolution mass spectrum depicting identification of automotive grease from post-blast residues extracted with hexane from IED \#10 rubber substrate fragment. Collected in positive mode. 
Identification of the oxidizer component of the binary explosives mixtures using DART-MS was also studied. Table 1 lists common examples of inorganic salts used as oxidizers in the production of homemade binary explosives. DART-MS analysis of these exemplary commercial sources of oxidizers was performed in negative mode. Instrument and solvent blanks were included to monitor cleanliness of the instrument and to identify background ions endogenous to the DART itself (Figure 51 and 52). Analysis of the commercial sources of oxidizers was included to determine detection capability for the real substances that are used to make HME. Ammonium nitrate sourced from instant cold compression packs, was identified by the observing the nitrate ion $\left(\mathrm{m} / \mathrm{z} 61.9867\left[\mathrm{NO}_{3}\right]^{-}\right)$ and its adduct with nitric acid $\left(\mathrm{m} / \mathrm{z} 124.9819\left[\mathrm{HNO}_{3}+\mathrm{NO}_{3}\right]^{-}\right)$. Potassium nitrate sourced from commercial stump remover, was identified by observing the nitrate ion $(\mathrm{m} / \mathrm{z}$ $\left.61.9867\left[\mathrm{NO}_{3}\right]^{-}\right)$. Another ion was also reproducibly observed at $\mathrm{m} / \mathrm{z} 121.9819\left(\left[\mathrm{C}_{7} \mathrm{H}_{5} \mathrm{O}_{2}\right]^{-}\right)$ which is attributable to an organic additive present in the product. Detection of nitrate inorganic salts was in agreement with expectation because of their sufficient vapour pressure at room temperature. ${ }^{12}$ Upon analysis of aqueous extracts collected from fragments recovered for IED \#10 post-blast, the same characteristic ions observed were observed identifying the oxidizer component of the binary HME. Analysis of the fragments from the remaining devices (\#7, 8 and 12$)$ will be completed in future work. 
Analysis of perchlorates and chlorates via DART-MS proved to be challenging, in agreement with other reported studies. ${ }^{67}$ The low vapour pressure of chlorates and perchlorates has been cited as the cause for poor ability to detect, which poses a challenge to thermal desorption required for the DART ionization process. ${ }^{67}$ Upon analysis of chlorate and perchlorate salts with the DART probe temperature of $250^{\circ} \mathrm{C}$, which is standard for analysis of explosives, detection of perchlorates and chlorates was not observed. No ions characteristic of perchlorate or chlorate were observed in any EICs generated from analysis of the binary explosive residues. Increasing the temperature of the DART ionizing gas has been reported as ineffective to recover either chlorate or perchlorate. ${ }^{67}$ Further method optimization to use DART-MS to detect either chlorate or perchlorate salts from post-blast binary explosive residues was not performed within the scope of this study presented, but will be explored with future work.

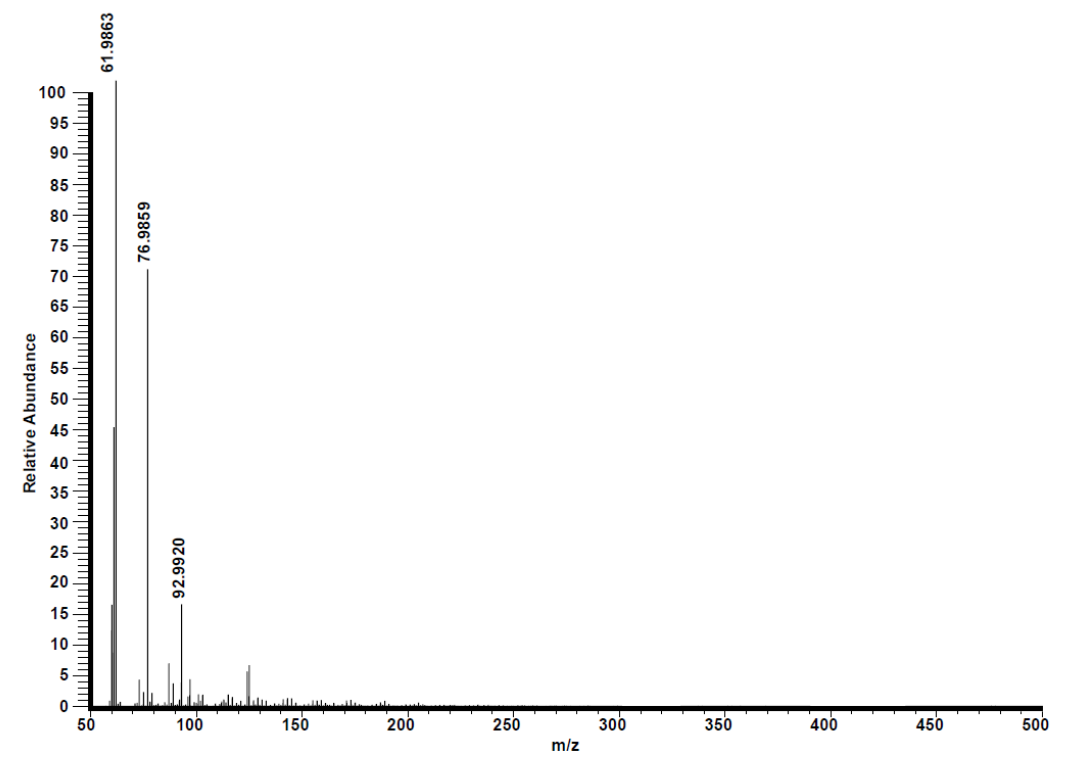

Figure 51. Negative mode, full scan high resolution mass spectrum upon operation of the DART-MS, depicting the endogenous ions. Total ion count $10^{6}$. 


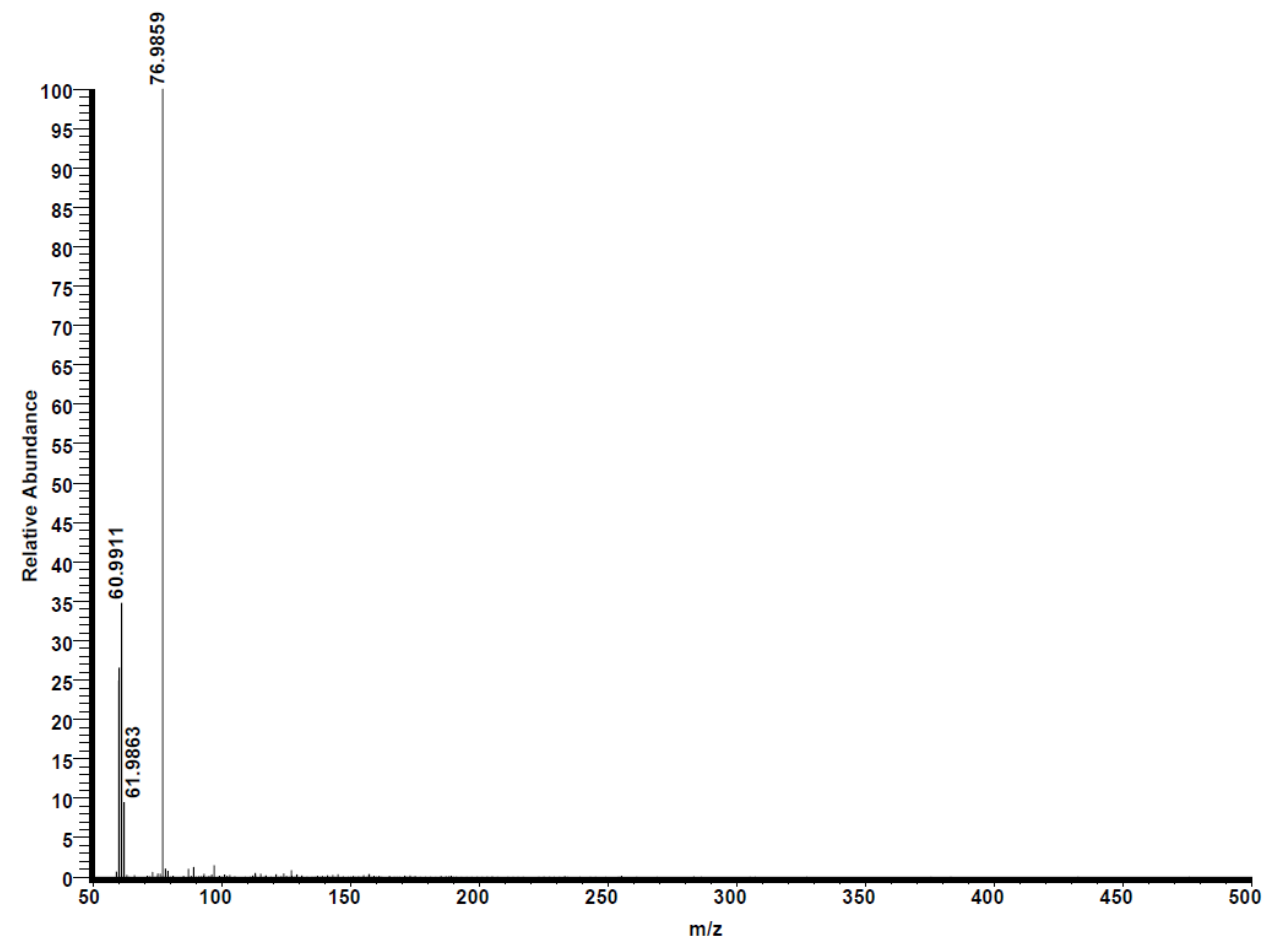

Figure 52. Analysis of water deposited onto a QuickStrip, in negative mode using full scan. Total ion count $10^{6}$.

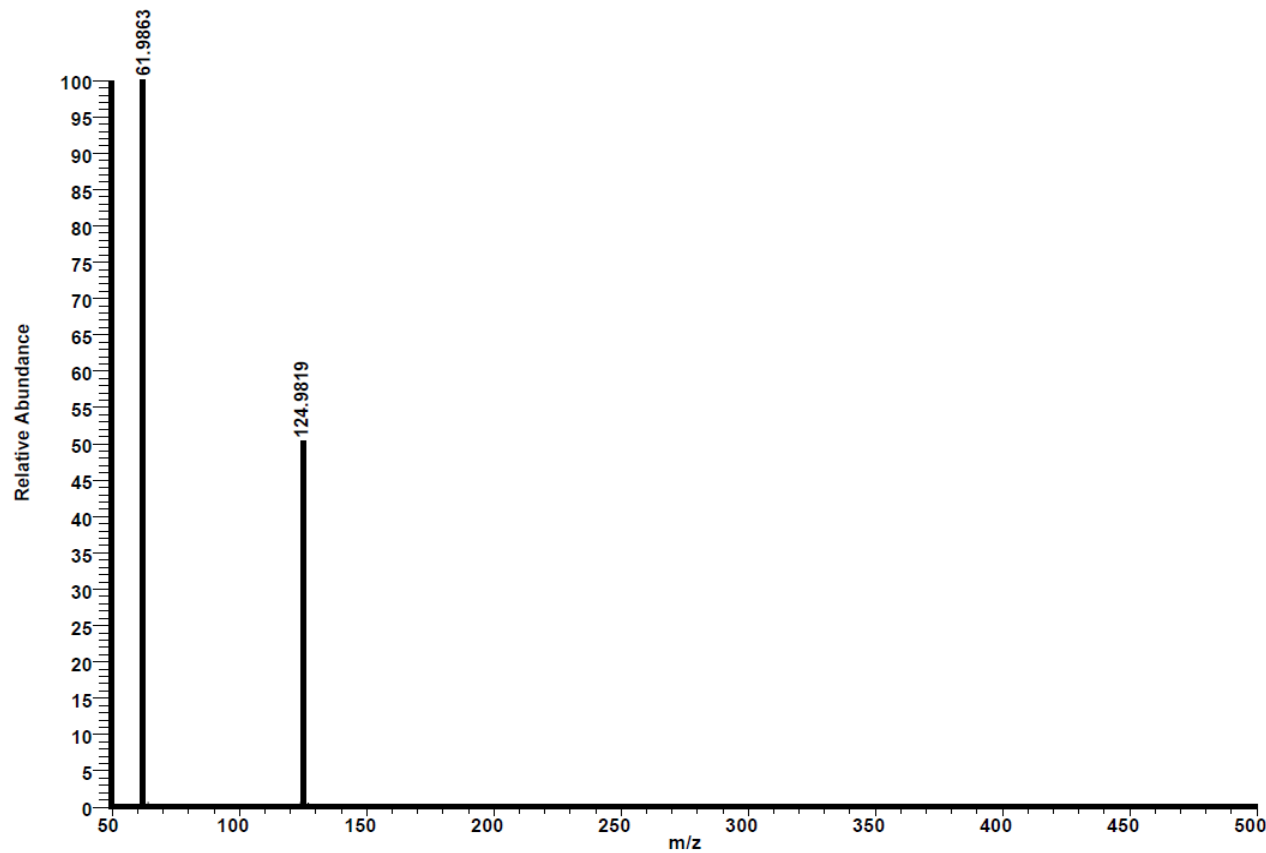

Figure 53. Negative mode, full scan high-resolution mass spectrum for ammonium nitrate analyzed as a reference material. 


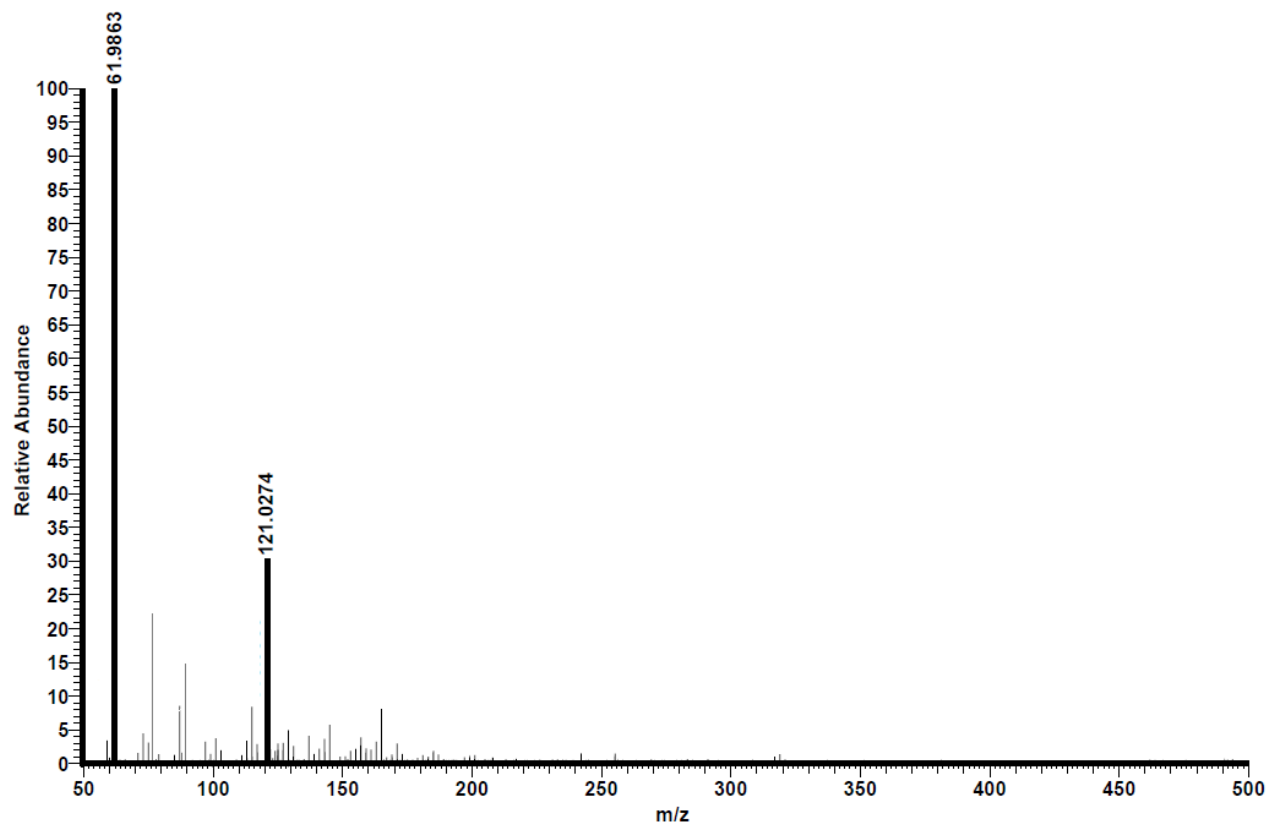

Figure 54. Negative mode, full scan high-resolution mass spectrum for a commercially available stump remover (commercial source of $\mathrm{KNO}_{3}$ ), analyzed as a reference material.

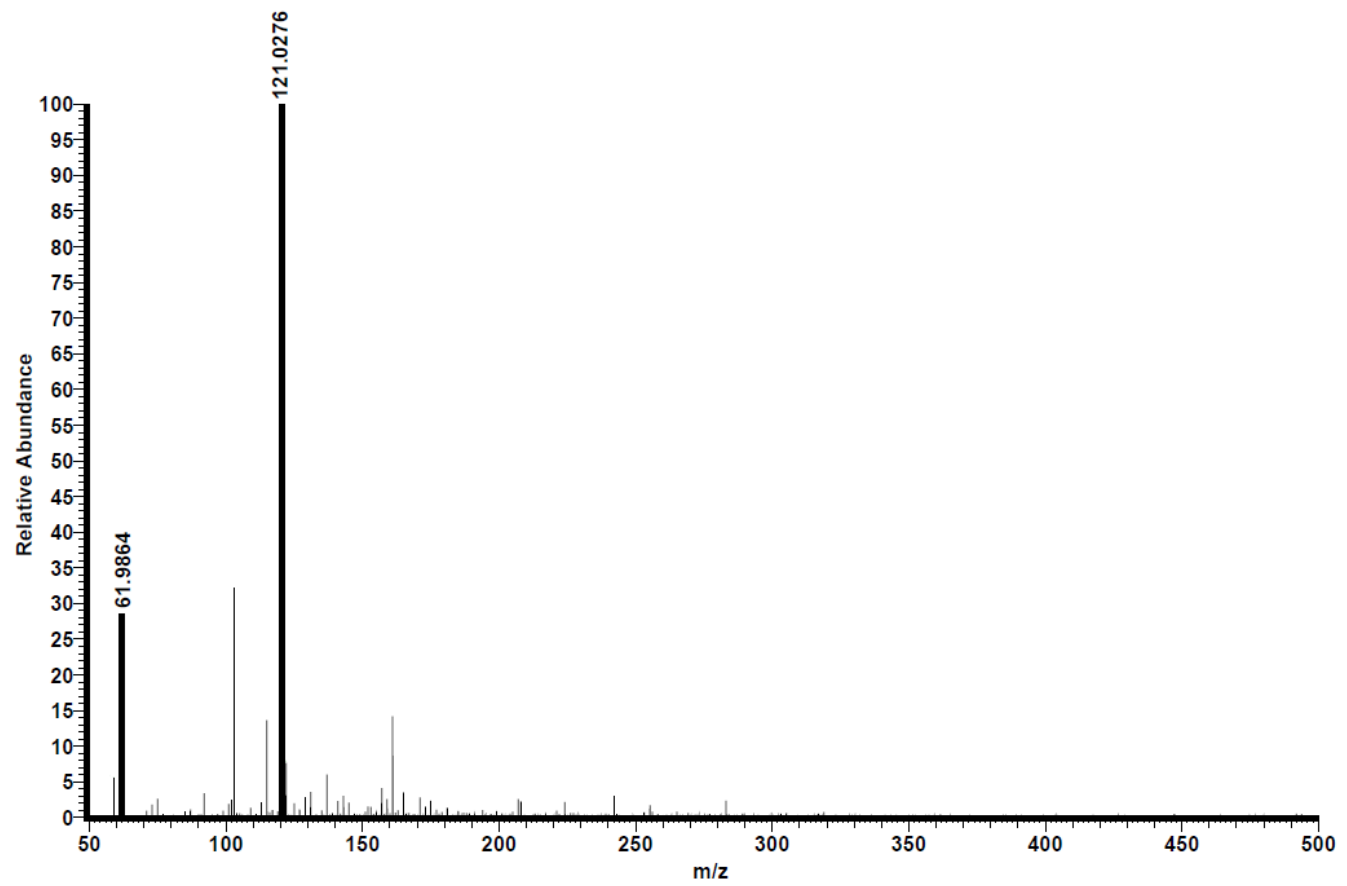

Figure 55. Identification of $\mathrm{KNO}_{3}$ in negative mode via full scan high resolution DART-MS analysis of post-blast residues extracted from metal substrate fragments from IED \#10 with water. 


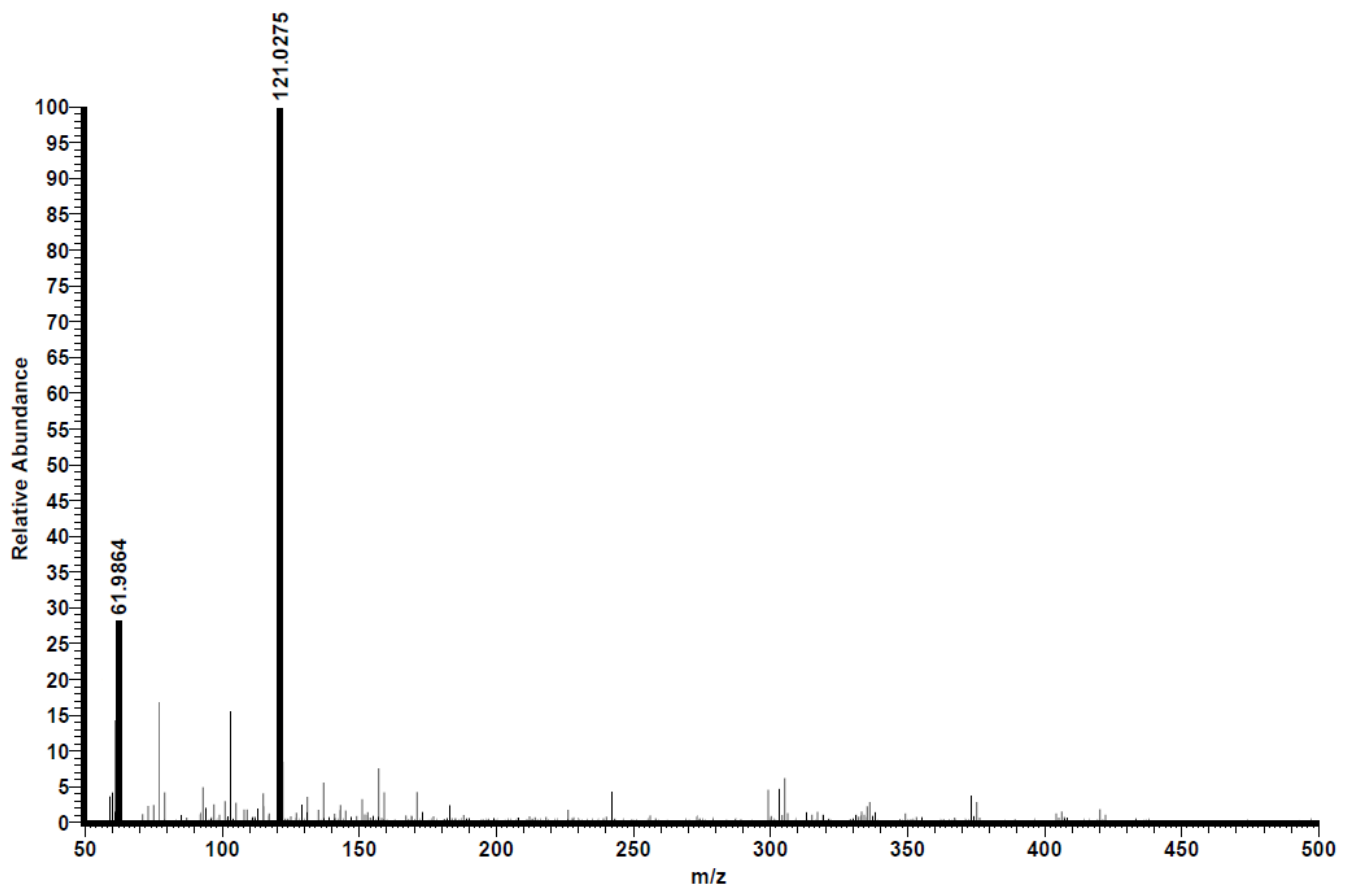

Figure 56. Identification of $\mathrm{KNO}_{3}$ in negative mode via high-resolution DART-MS analysis of post-blast residues extracted from plastic substrate fragments from IED \#10 with water.

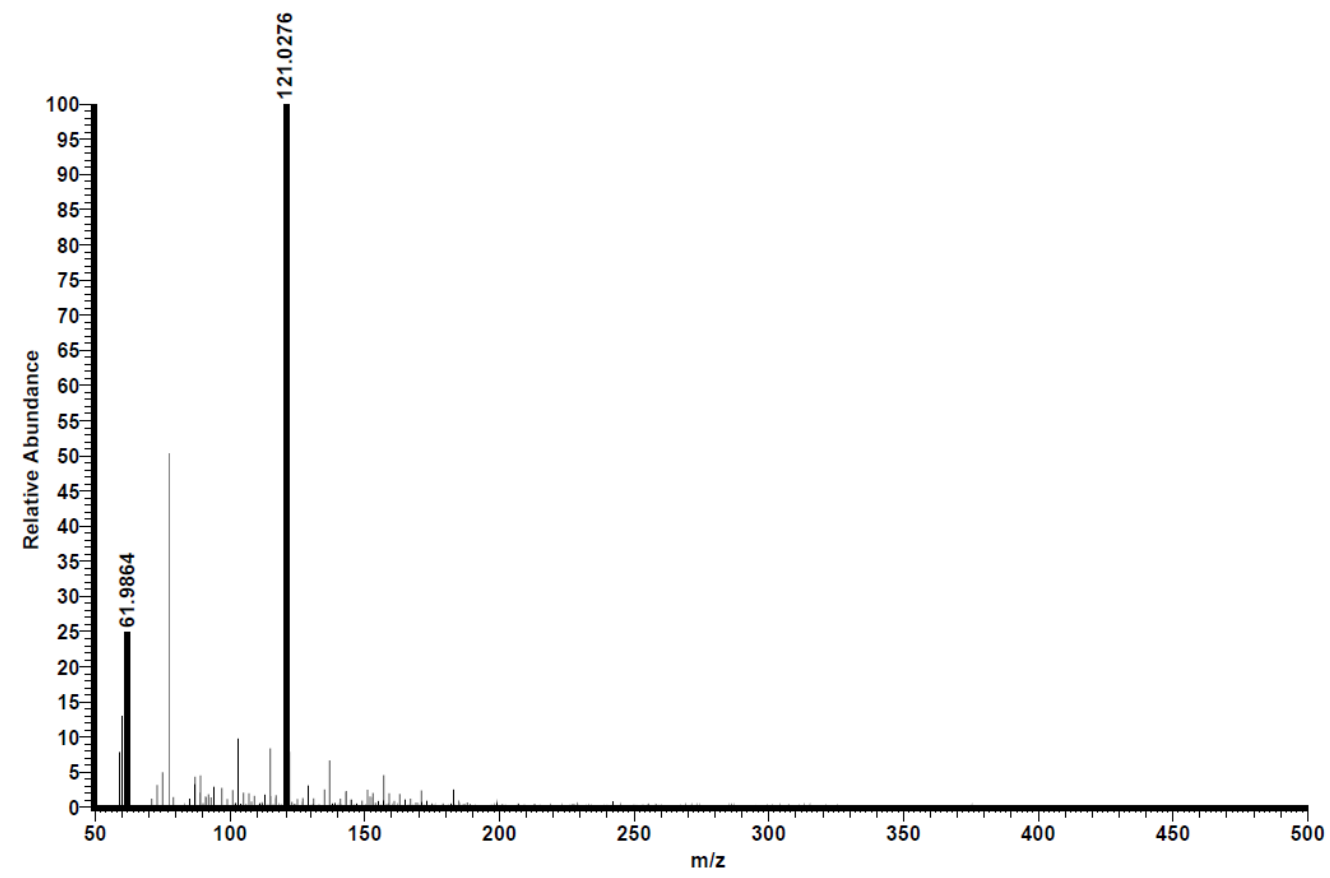

Figure 57. Identification of $\mathrm{KNO}_{3}$ in negative mode via full scan high-resolution DART-MS analysis of post-blast residues extracted from rubber substrate fragments from IED \#10 with water. 


\subsubsection{Smokeless Powders}

As blast effects (heat and pressure) can change the composition of a compound, ignition of NC is expected to result in thermal breakdown to oligomers of varying length and degree of nitration. Characterization of these thermal breakdown products is challenging as reference materials do not exist. Commercial sources of monomer or dimer nitrated sugar products are not available motivating the synthesis of a reference material in-house. Successful synthesis of $\beta$ - cellobiose octanitrate (ONCB) afforded a reference material structurally similar to potential breakdown products from the thermal degradation of NC.

Initial analysis of ONCB and unconsumed grains of single and double based smokeless powder resulted in dissimilar spectra. Ions characteristic of the additives and preservatives included in smokeless powder (e.g. DPA and EC) dominated the spectra obtained upon analysis of both single and double base products unconsumed. Predominant ions observed upon analysis of ONCB were absent in the spectra obtained via analysis of the smokeless powder. Comparison of ONCB and residues collected from the ignited smokeless powder also did not warrant similar spectra. New ions were observed in the spectra from analysis of the ignited smokeless powders but were dissimilar from the predominant ions in the ONCB. These results suggested that the ONCB in pure form may not be a comparable material for the characterization of NC thermal breakdown products. 
Comparison of ignited ONCB and smokeless powder was completed to determine if the ignited product was a more suitable reference material than unburned ONCB. Analysis of extracted ONCB and smokeless powder residues with acetonitrile was completed. After subtracting the spectral contributions from acetonitrile (Figure 23), similar ions in the $\mathrm{m} / \mathrm{z}$ range of $300-500$ were observed upon analysis of ONCB and smokeless powder residues. Proposed characteristic ions are as follows; $\mathrm{m} / \mathrm{z} 323.0718$, 341.0822, 491.0744, 508.0643, and 536.0597. Ion abundance was highly variable across analyses of all samples. The highly complex sample matrix created by burning the products resulted in mass spectra with ions observed from $\mathrm{m} / \mathrm{z} 50-500$ at counts of $10^{6}$ $-10^{7}$. Subtraction of spectral contribution from the solvent reduced ion counts by only a signal magnitude $\left(10^{7}\right.$ to $\left.10^{6}\right)$ further emphasizing complexity of the ignited samples. Figure 58 depicts how EICs were used to confirm that the proposed characteristic ions are not attributed to any sample component other than nitrated sugar thermal breakdown products. The ions used to produce the EICs are indicated on the left of the figure. Descriptors of the samples placed in each well on a Quickstrip are labelled along the top. The long black box with grey circles overlaid on the EIC depicts the Quickstrip card that blank, control and extract samples were deposited onto. The first well contained the extraction solvent; included to facilitate background subtractions. Blank wells were analyzed between each sample to facilitate subtraction of ions endogenous to the DARTMS and monitor instrument cleanliness. Analysis of TNT served as a negative control as the thermal breakdown products would not be present in the TNT sample and their structure would not be similar to that of TNT. The ignited residues were deposited onto 
wells five, seven and nine. Observation of the characteristic ions in the EIC for all three samples confirms detection and identification of similar thermal breakdown products between ignited ONCB, SB and DB smokeless powders. Absence of the characteristic ions in the wells associated with the instrument background, solvent, and TNT further confirms association to the nitrated sugar thermal breakdown products. Characterization of the thermal breakdown products is supported by identification of common characteristic ions in both the reference sample (ignited ONCB) and the consumed smokeless powders.

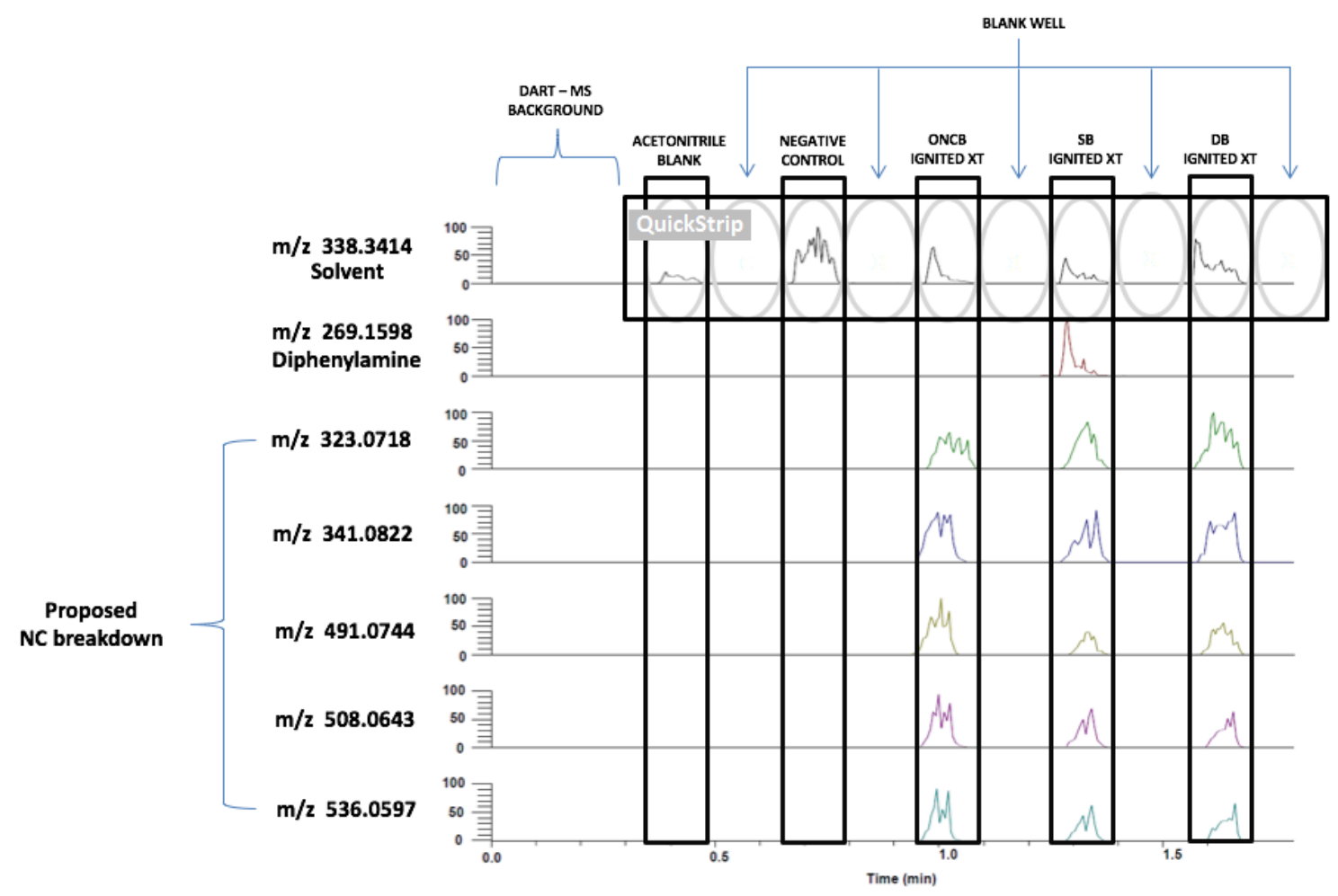

Figure 58. Determination of ions characteristic of the thermal breakdown products. 
Mass analysis of each ion was used for structure determination based on acceptable mass shift ( $\pm 0.003 \mathrm{amu}$ ) with respect to high-resolution accurate mass measurements (Table 6). MS/MS analysis may provide further clarification on the structure of the thermal breakdown products by producing fragments that can be used to elucidate functional groups and stereochemistry, and shall be explored in future work.

Table 6. List of mass formulae for the ions characteristic of nitrated sugar thermal breakdown products, with associated mass shift (amu).

\begin{tabular}{|c|c|c|}
\hline $\mathrm{m} / \mathrm{z}$ & Mass Formulae & Mass shift (amu) \\
\hline \multirow{2}{*}{323.0718} & $\mathrm{C}_{9} \mathrm{H}_{9} \mathrm{O}_{5} \mathrm{~N}_{9}$ & -0.486 \\
\cline { 2 - 3 } & $\mathrm{C}_{10} \mathrm{H}_{15} \mathrm{O}_{10} \mathrm{~N}_{2}$ & -0.491 \\
\hline \multirow{3}{*}{341.0822} & $\mathrm{C}_{9} \mathrm{H}_{11} \mathrm{O}_{6} \mathrm{~N}_{9}$ & -0.611 \\
\cline { 2 - 3 } & $\mathrm{C}_{10} \mathrm{H}_{17} \mathrm{O}_{11} \mathrm{~N}_{2}$ & -0.616 \\
\hline \multirow{3}{*}{491.0744} & $\mathrm{C}_{13} \mathrm{H}_{15} \mathrm{O}_{13} \mathrm{~N}_{8}$ & -1.129 \\
\cline { 2 - 3 } & $\mathrm{C}_{26} \mathrm{H}_{11} \mathrm{O}_{7} \mathrm{~N}_{4}$ & -0.616 \\
\hline \multirow{3}{*}{508.0643} & $\mathrm{C}_{12} \mathrm{H}_{14} \mathrm{O}_{14} \mathrm{~N}_{9}$ & -1.112 \\
\cline { 2 - 3 } & $\mathrm{C}_{26} \mathrm{H}_{12} \mathrm{O}_{8} \mathrm{~N}_{4}$ & -0.605 \\
\hline \multirow{2}{*}{536.0597} & $\mathrm{C}_{13} \mathrm{H}_{14} \mathrm{O}_{15} \mathrm{~N}_{4}$ & -0.907 \\
\cline { 2 - 3 } & $\mathrm{C}_{27} \mathrm{H}_{12} \mathrm{O}_{9} \mathrm{~N}_{4}$ & -0.399 \\
\hline
\end{tabular}

Solubility of the thermal breakdown products was explored using a series of solvents commonly used in accepted forensic practices (acetone, $\mathrm{MeOH}$, water, acetonitrile, DCM and hexane). Changes in observation of ions in the EICs was used as a measurement of solubility. The thermal breakdown products are assumed to be relatively polar if they retain the polar nitrate groups. Solvents of varying polarity were used to confirm this hypothesis. Figure 59 depicts a combination of the EICs obtained from analysis of ONCB, SB and DB residues extracted with six different solvents. Wells highlighted by the red box indicates samples extracted with hexane. Absence of all 
characteristic ions upon analysis of hexane extract confirms the thermal breakdown products are not soluble in a non-polar solvent. Wells highlighted in the purple box indicate samples extracted using DCM. Characteristic ions were observed upon analysis of ignited ONCB but not smokeless powders confirming many species with slightly different polarity could be present. Yellow, green and orange boxes indicate extraction with acetone, $\mathrm{ACN}$ and $\mathrm{MeOH}$, respectively. As these are relatively polar solvents, with retention of the nitrate groups upon thermal degradation, it is not surprising that residues were soluble in these polar solvents. The blue box depicts extraction with water and absence of characteristic ions confirms they are not soluble in water.

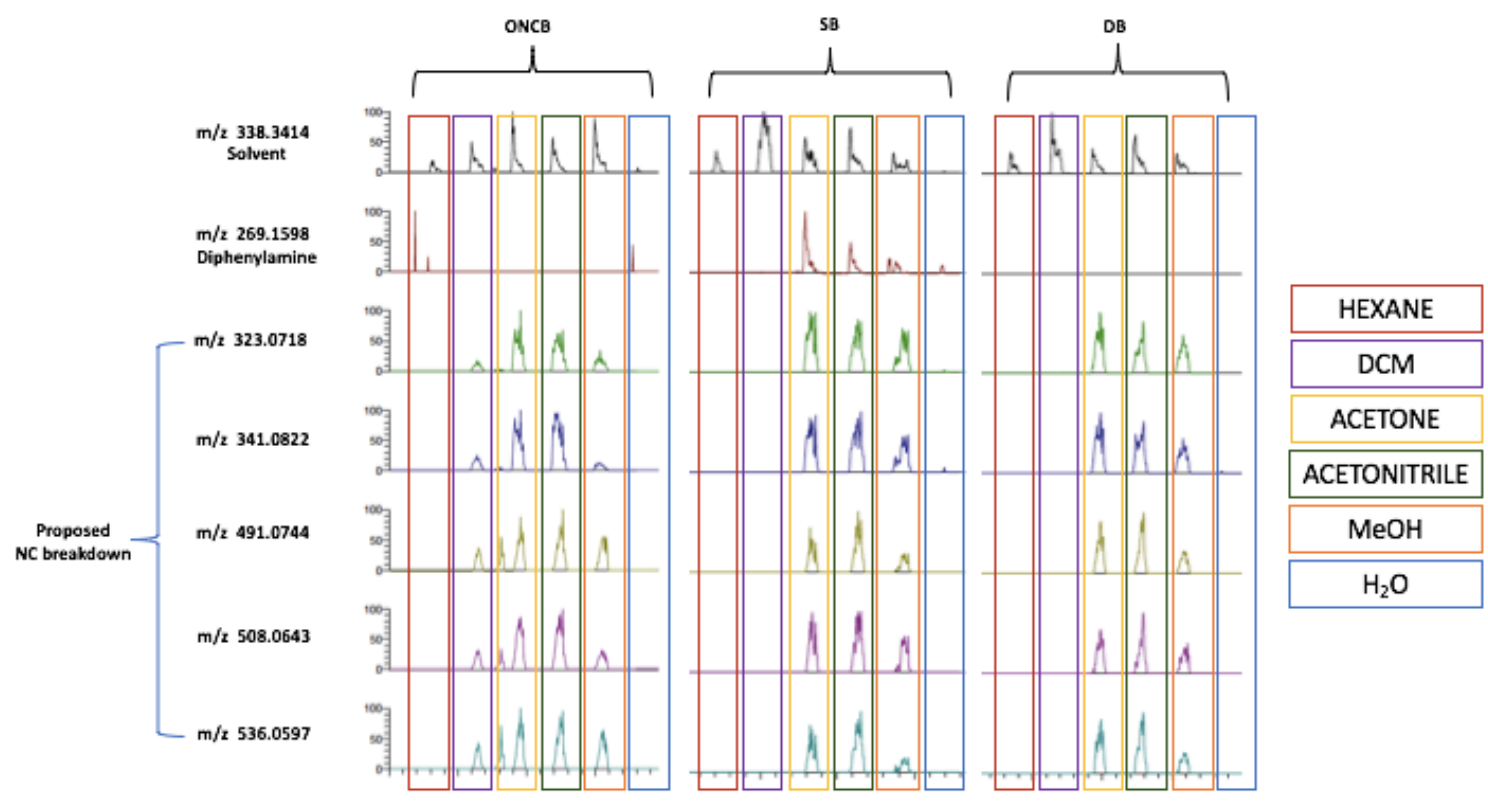

Figure 59. Relative solubility of residues containing thermal breakdown products. 
Synthesis of a reference material such as ONCB was essential for detection and identification of NC thermal breakdown products. Ignition of ONCB afforded production of residues alike those produced upon the ignition of a smokeless powder. Successful detection and identification of similar ions upon analysis of ignited products provides mechanisms for characterization of thermal breakdown products. Post-blast identification of NC would remain impossible without detection and identification of characteristic thermal breakdown products.

Future goals include using the ignited ONCB as a reference material and DART-MS to fully characterize smokeless powders from post-blast residues when used in IED. Therefore, it is imperative to test applicability of this novel work with real post-blast fragments. These tests will be carried out in future work to ensure that characteristic ions observed upon burning the products are identified upon analysis of post-blast residues. To ensure efficient extraction post-blast residues should be extracted with ACN. 


\section{Conclusion}

DART coupled with high-resolution MS was demonstrated as a suitable analytical technique applicable for forensic identification of homemade explosive from post-blast residues recovered from IED fragments representative of items and substrates commonly recovered from genuine bombing investigations. Explosives of interest studied included organic peroxides (triacetone triperoxide, hexamethylene triperoxide and methyl ethyl ketone peroxide), binary explosives (fuel and oxidizer mixtures) and commercial smokeless powders (both single- and double-base). Each explosive was characterized by comparing questioned mass spectra with known reference materials to identify ions characteristic of the target explosive. DART-MS was also successfully used to characterization the thermal breakdown products of nitrocellulose (i.e. nitrated sugar derivatives) which can be used to identify a smokeless powder post-blast in the absence of recovering an intact grain.

DART-MS was verified to identify HME residues by direct analysis of the post-blast IED fragments themselves and by indirect analysis of sub-samples collected using dry or wet swabs with solvents. Dry cotton swabs were found effective for explosive residue recovery and yielded the least co-extracted environmental background. Since dry swabbing is also a commonplace sampling technique used at security check-points to detect concealed explosives or residues, we suggest DART-MS is compatible for rapid forensic detection of trace explosives in screening applications. 


\section{Future Work}

The residues studied in this project were not subjected to weathering or degradation effects which are commonly observed in forensic samples collected from crime scenes as this was beyond the scope of the study. Post-blast fragments were collected and packaged in sealable nylon-evidence bags immediately after detonation unlike realistic scenarios where many safety precautions are taken before front-line members can enter a blast scene to collect evidence. Exposure to environmental factors, such as precipitation, temperatures changes or sunlight (e.g. UV) can affect the lifetime and recoverability of explosive residues post-blast. The potential for sample loss or contamination of evidence (e.g. sourced by exposure to weather) could be studied in the future to further replicate realistic scenarios. Delayed collection of the fragments in time increments representative of realistic scenarios is one way to study exposure effects. Simulating changes in temperature by exposing the fragments to heat (e.g. placing in an oven with temperature set to represent realistic Canadian summer heat) is another mechanism in which measuring the effects of weathering could be studied.

Furthermore, due to the homemade nature of OPBE and binary mixtures different precursor sources and synthesis methods can contribute to changes in the residues collected post-blast. Additional analysis of post-blast residues from detonation of devices that contain products synthesized with different precursors (i.e. lower wt \% hydrogen peroxide, different acid catalysts, and additional commercial fuel and oxidizer sources) would further depict the robust capability of DART-MS as an identification method for homemade explosives. 


\section{References}

[1] The Global Practice of Forensic Science, edited by Douglas H. Ubelaker, John Wiley \& Sons, Incorporated, 2014.

[2] R.W. Byard, H. James, J. Berketa, K. Heath. J Forensic Sci (2016) 61: 545-547.

[3] N. Abdul-Karim, C.S. Blackman, P.P. Gill, E. M.M. Wingstedt, B.A.P. Reif. RSC Adv. 4 (2014) $54354-54371$.

[4] J. S. Caygill, F. Davis, S P. J. Higson. Talanta (2012) 88: 14-29.

[5] Eiceman GA, Karpas Z. Ion mobility spectrometry, 2nd rev. edn. Boca Rotan, FL: Taylor and Francis, 2005.

[6] G.W. Cook, P.T. LaPuma, G.L. Hook, B.A. Eckenrode. Journal of Forensic Science (2010) 55: 1582-1591.

[7] C. L. Crawford, H.H. Hill, Jr. Analytica Chimica Acta (2013) 795: 36-43.

[8] Beveridge, Forensic Investigations of Explosions, CRC Press Taylor \& Francis Group, India, 2012.

[9] D. DeTata, P. Collins, A. McKinley. Forensic Science International (2013) 233: 6374.

[10] X. Xu, M. Koeberg, C.-J. Kuijpers, E. Kok. Science and Justice (2014) 54: 3-21.

[11] T.P. Forbes and E. Sisco. Analyst (2018) 143: 1948-1969.

[12] J. Pavlov, A. B. Attygalle. Anal. Chem. (2013) 85: 278-282.

[13] E. Sisco, J. Dake, C. Bridge. For. Sci. Int. (2013) 232: 160-168.

[14] T.P. Forbes, E. Sisco, M. Staymates, G. Gillen. Anal. Methods (2017) 9: 49884996.

[15] Kubota, Propellants and Explosives: Thermochemical Aspects of Combustion, 2nd ed., Wiley-VCH, Germany, 2007.

[16] J. C. Oxley, J. L. Smith, J. Huang, W. Luo. J. Forensic Sci (2009) 54: 1029-1033.

[17] L. R. Rothstein and R. Petersen. Propellants and Explosives (1979) 4: 50-60.

[18] M. Vaullerin and A. Espagnacq. Proppelants, Explosives and Pyrotechnics. (1998) 23: 237-239.

[19] N.Hagan, I. Goldberg, A. Graichen, A. St. Jean, C. Wu, D. Lawrence, P. Demirev. J. Am. Soc. Mass Spectrom. (2017) 28: 1531-1539. 
[20] R.D. Bach, P. Y. Ayala, H. B. Schlegel. J. Am. Chem. Soc. (1996) 118: 12758-12765.

[21] Agrawal, J. P.; Hodgson, R. D. Organic Chemistry of Explosives (2007).

[22] J. Pachman, R. Matyas. Forensic Science International (2011) 207: 212-214.

[23] R. vs. BRIDGES, Dane. Ontario Court of Justice; Ottawa: 2012 - 2013.

[24] R. vs. AMSEL, Guido. Provincial Court of Manitoba; Winnipeg: 2017 - 2018.

[25] F. S Romolo, L. Cassioli, S. Grossi, G. Cinelli, M.V. Russo. Forensic Sci. Int. (2013) 224: 96-100.

[26] N.A. Milas, A. Golubovic. J.A.C.S. (1959) 81: 5824-5826.

[27] A. Miyake, K. Takahara, T. Ogawa, Y. Ogata, Y. Wada and H. Arai. Jour. of Loss Prevention in the Pro. Ind. (2001) 14: 533-538.

[28] R. M. Heramb,B. R. McCord. For. Sci. Comms. (2002) 4.

[29] R. Gonzalez-Mendez, C. A. Mayhew. J. Am. Soc. Mass Spectrom. (2017) 30: 15311539.

[30] C. Christodoulatos, T. Su, A. Koutsospyros. Water Environ Res. (2001) 27 : 185191

[31] E. Goudsmits, G. Sharples, J.W. Birkett. TrAC Trends Anal. Chem. (2015) 74: 4657

[32] R.V. Taudte, A. Beavis, L. Blanes, N. Cole, P. Doble, C. Roux. Biomed. Res. Int. (2014) 16: 16.

[33] M. Joshi, K. Rigsby, J.R. Almirall. Forensic Sci. Int. (2011) 208: 29-36

[34] M. Mach, A. Pallos, P. Jones. J. Forensic Sci. (1978) 23: 433-445

[35] A. Zeichner, B. Eldar, B. Glattstein, A. Koffman, T. Tamiri, D. Muller J. Forensic Sci. (2003) 48: 961-972

[36] R. Ewing. Talanta 54 (2001) 515-529.

[37] Beveridge, Forensic Investigations of Explosions, CRC Press Taylor \& Francis Group, India, 2012.

[38] O. Coskun, North Clin Istanb. (2016) 3: 156-160.

[39] D.S. Moore, J.V. Goodpaster. Anal Bioanal Chem (2009) 395: 245-246

[40] Barron and E. Gilchrist, Analytica Chimica Acta (2014) 806: 27-54.

[41] E. Gilchrist, N. Smith, L. Barron, Analyst (2012) 137. 
[42] J. M. Nilles, T. R. Connell, S. T. Stokes. Propellants Explos. Pyrotech. (2010) 35: 446-451.

[43] K. Clemons, J. Dake, E. Sisco, G.F. Verbec IV. Forensic Sci. Int. (2013) 231: 98-101.

[44] L. Frederick, T. Joseph, B.D. Muselman, A.B. Science and Justice (2016) 56: 321328.

[45] F. Rowell, J. Seviour, A. Y. Lim, C. G. Elumbaring-Salazar, J. Loke, J. Ma. Forensic Sci Int (2012) 221: 84-91.

[46] J.R. Swider. Journal of Forensic Sciences (2013) 58: 1601-1606.

[47] E. Sisco, J. Dake, C. Bridge. Forensic Sci. Int. (2013) 232: 160-168.

[48] F. Rowell, J. Seviour, A. Y. Lim, C. G. Elumbaring-Salazar, J. Loke, J. Ma. Forensic Sci. Int. (2012) 221: 84-91.

[49] Dong, Direct Analysis in Real Time Mass Spectrometry, Wiley-VCH, Germany, 2018.

[50] D. C. Harris et al, in "Quantitative Chemical Analysis." $7^{\text {th }}$ edition. W.H. Freeman and Company. NY. USA. 2007.

[51] M. Huang, C. Yuan, S. Cheng, Y. Cho, J. Shiea. Annu. Rev. Anal. Chem. (2010) $3: 43-65$.

[52] A. G. Marshall and C.L. Hendrickson. Annu. Rev. Anal. Chem. (2008) 1: 579-99.

[53] Cody, R. B.; Laramée, J.; Nilles, J. M. Anal. Chem. (2005) 77: 2297-2302.

[54] E. S. Chernetsova, G. E. Morlock. Mass Spectrometry Reviews (2011) 30: 875883.

[55] J. M. Nilles, T. R. Connell, H. D. Durst. Anal Chem (2009) 81 : 6744-6749.

[56] Michalski, A. et al. Mol. Cell. Proteomics (2011) 9.

[57] Brunnee, C. Int. J. Mass Spectrom. Ion Proc (1987) 76: 125-237.

[58] J.V. Olsen, B. Macek, O. Lange, A. Makarov, S, Horning, M, Mann. Nature Methods (2007) 4: 709-712.

[59] Hardman, M., and Makarov, A. A. Anal. Chem. (2003) 75: 1699 -1705.

[60] J.C. Oxley, J.L. Smith, K. Shinde, J. Moran. Propellants, Explos., Pyrotech. (2005) 30: $127-130$.

[61] L.E. DeGreet, M.M. Cerreta, C.J. Katille. Forensic Chem. (2017) 4: 41-50. 
[62] T.A. Ong, T. Mendum, G. Geurtsen, J. Kelley, A. Ostrinskaya, R. Kunz. Anal. Chem. (2017) 89: 6482-6490.

[63] N. Abdul-Karim, R. Morgan, R. Binions, T. Temple, K. Harrison. J. Forensic Sci. (2013) 58: 365-371.

[64] D.S. Saang'onyo, D.L. Smith. Rapid Commun. Mass Spectrom. (2012) 26: 385391.

[65] R.J. Brownlow, K.E. Dagnall, C.E. Ames. J. Forensic Sci. (2012) 57: 713-717.

[66] E. Stauffer, J. Dolan and R. Newman. Fire Debris Analysis, Elsevier, 2008.

[67] T.P. Forbes, E. Sisco, M. Staymates. Anal. Chem. (2018) 90: 6419-6425. 


\section{Appendix 1: Supplementary Information}

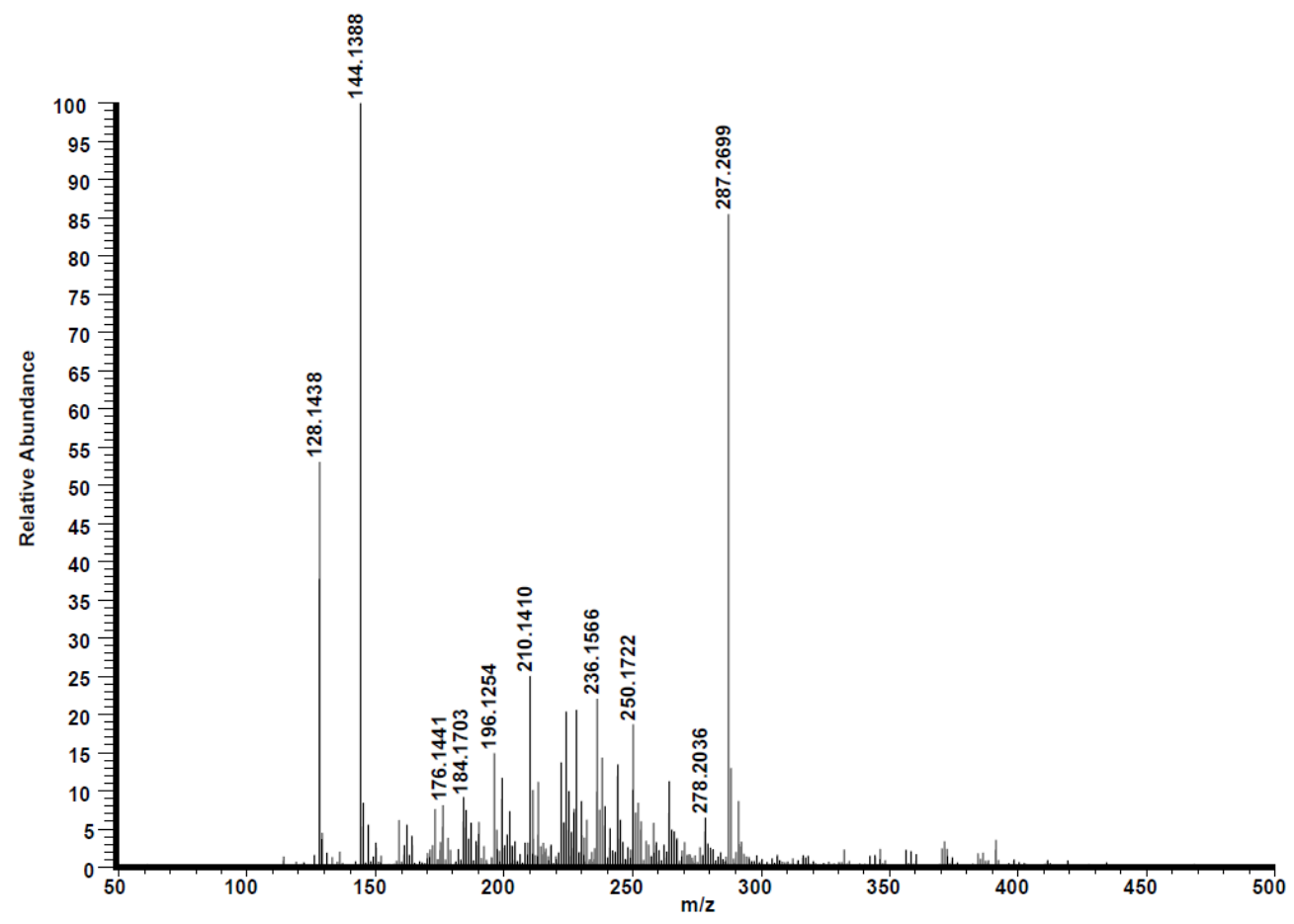

Figure 60. Positive mode, full scan: high-resolution mass spectra for diesel, analyzed as a reference material

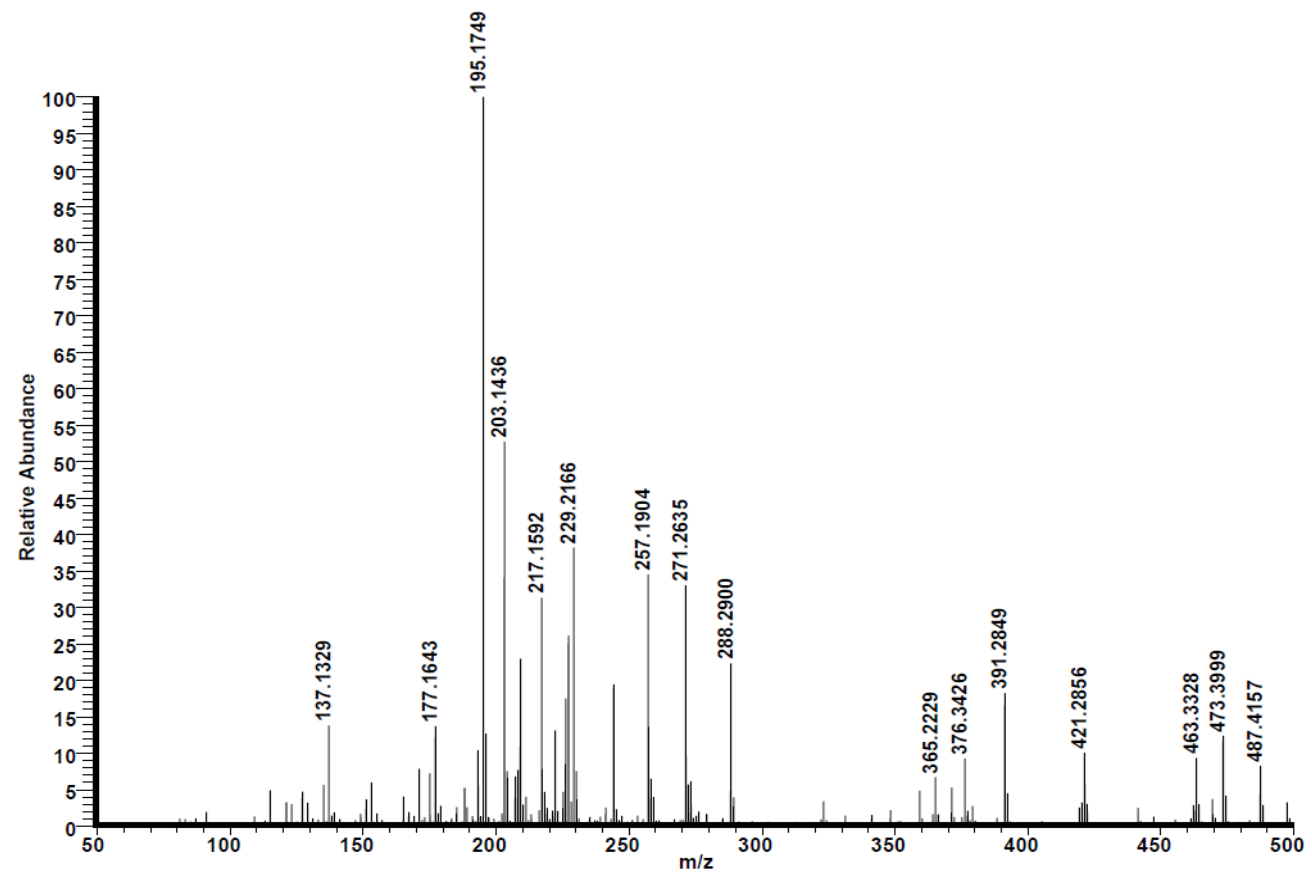

Figure 61. Positive mode, full scan: high-resolution mass spectra for lamp oil, analyzed as a reference material 


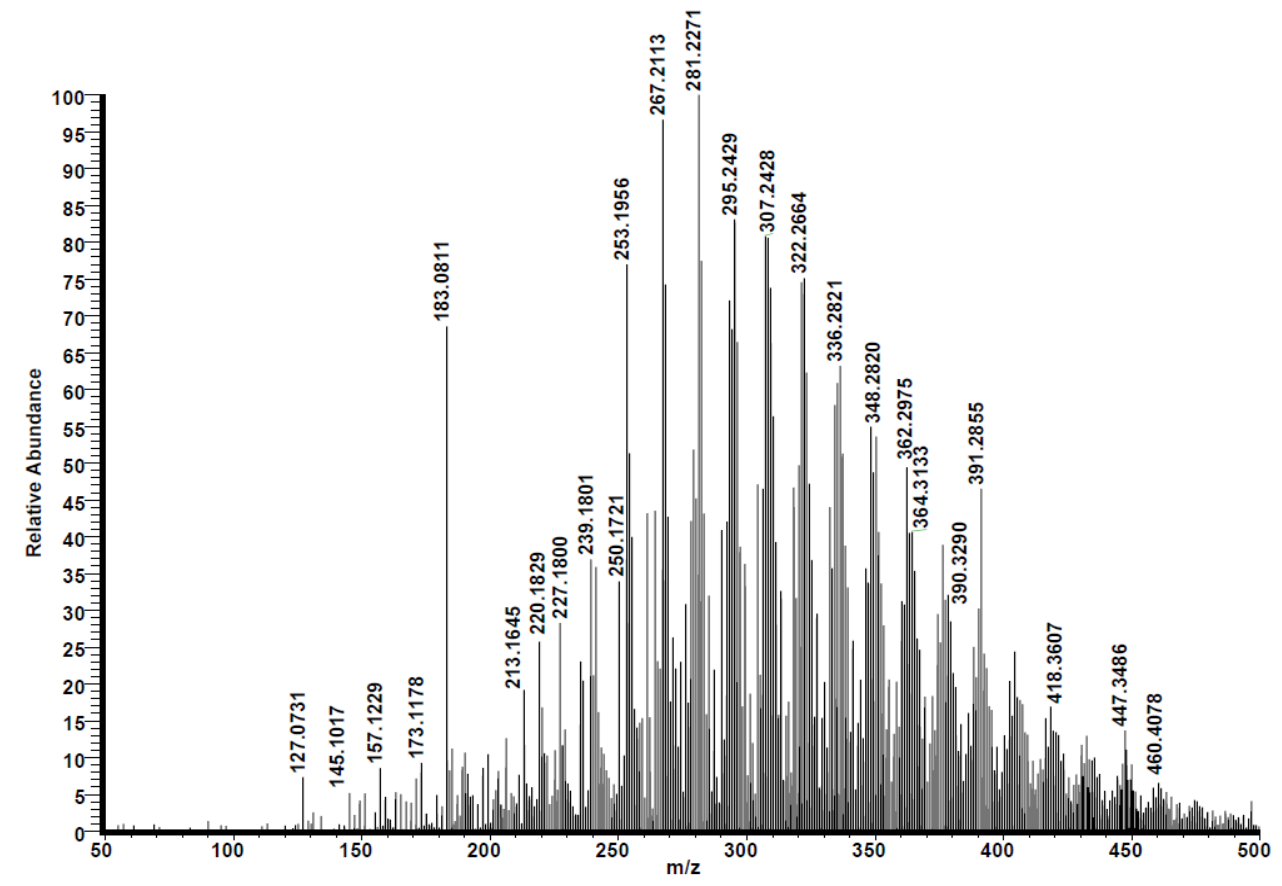

Figure 62. Positive mode, full scan: high-resolution mass spectra for Vaseline, analyzed as a reference material

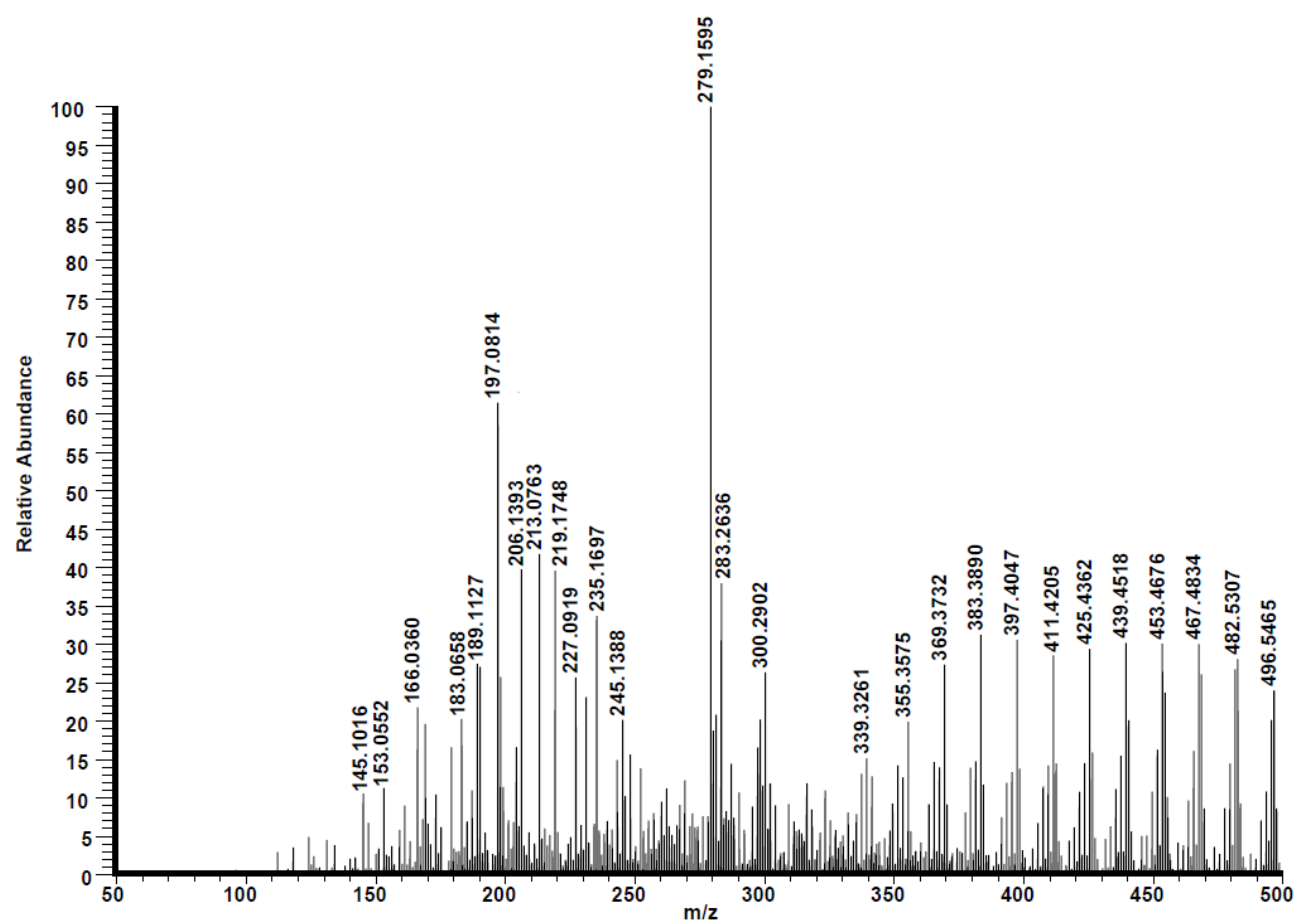

Figure 63. Positive mode, full scan: high-resolution mass spectra for wax, analyzed as a reference material 